\title{
WestVirginiaUniversity
}

THE RESEARCH REPOSITORY @ WVU

Graduate Theses, Dissertations, and Problem Reports

2004

\section{Models of high-temperature desulfurization using zinc-based sorbents}

Yong Zhang

West Virginia University

Follow this and additional works at: https://researchrepository.wvu.edu/etd

\section{Recommended Citation}

Zhang, Yong, "Models of high-temperature desulfurization using zinc-based sorbents" (2004). Graduate Theses, Dissertations, and Problem Reports. 1571.

https://researchrepository.wvu.edu/etd/1571

This Thesis is protected by copyright and/or related rights. It has been brought to you by the The Research Repository @ WVU with permission from the rights-holder(s). You are free to use this Thesis in any way that is permitted by the copyright and related rights legislation that applies to your use. For other uses you must obtain permission from the rights-holder(s) directly, unless additional rights are indicated by a Creative Commons license in the record and/ or on the work itself. This Thesis has been accepted for inclusion in WVU Graduate Theses, Dissertations, and Problem Reports collection by an authorized administrator of The Research Repository @ WVU. For more information, please contact researchrepository@mail.wvu.edu. 


\title{
Models of High Temperature Desulfurization Using Zinc Based Sorbents
}

\author{
Yong Zhang \\ Thesis Submitted to the \\ College of Engineering and Mineral Resources \\ at West Virginia University \\ in partial fulfillment of the requirements \\ for the degree of \\ Master of Science \\ in \\ Chemical Engineering \\ Department of Chemical Engineering \\ Morgantown, West Virginia \\ 2004
}

Keywords: Desulfurization, Zinc Based Sorbents, SCM, GPM

SEM, Particle Size Distribution 


\section{Abstract \\ Models of High Temperature Desulfurization Using Zinc Based Sorbents}

Yong Zhang

This study focuses on determining models that are currently available to describe gas-solid reactions to predict the observed (experimental) results from a series of TGA experiments. Variations of the GPM (Grainy Pellet Model) were used and shown to give good agreement with experimental conversion histories of zinc oxide particle undergoing sulfidation using $\mathrm{H}_{2} \mathrm{~S}$ at temperature in the range of 482 to 593 degree centigrade.

By using SEM (Scanning Electron Microscope) and Visilog $^{\mathrm{TM}}$ imaging analysis software, the grain size distribution and grain sphericity can be obtained.

The basic forms of the SCM (Shrinking Core Model) and GPM models could not predict correctly the observed conversion-time data for pure zinc oxide sulfidation reaction in this study. Modifications to the models include considering: the effect of conversion on the physical properties of the sorbent due to the difference in molecular volume of zinc oxide and zinc sulfide, grains size distribution, grain shape.

A bimodal size distribution of grains for the GPM was found to give the best match with the experimental data, but from particle SEM image, it was clear that there was a wide grain size distribution. Nevertheless, by using a distribution of between 10 and 30 grain size, the predicted fit was worse than the bimodal distribution. 


\section{Dedication}

To my wife, Mei, and my daughter, Raina,

Without them, nothing,

With them, everything matters.

I love you! 


\section{Acknowledgements}

I wish to express sincere appreciation to Dr. Richard Turton for providing direction and guidance throughout the years in the research. Through the intense dedication required to complete the research, I experienced great joys and great frustrations. During the impressive period of the research, I got much help from many sectors, and to this end I would like to thank the research group of Dr. Richard Turton, Dr. Charter Stinespring and David Berry, M.S. for providing an atmosphere of encouragement throughout the years.

I also want to express my gratitude to my parents and my wife for their support and encouragement. 


\section{Table of Content}

Ackstract

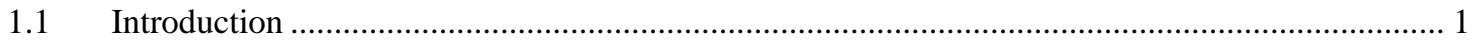

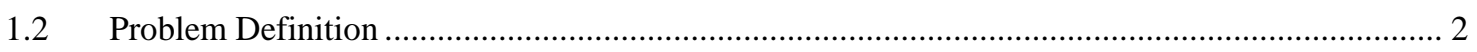

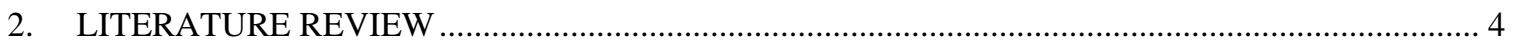

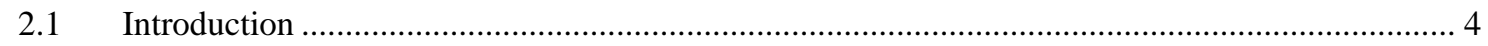

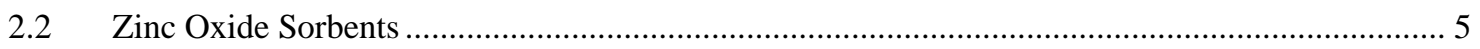

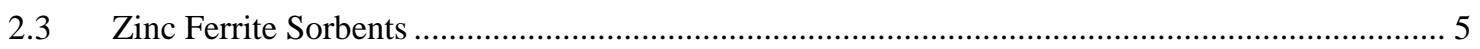

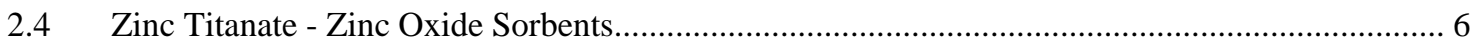

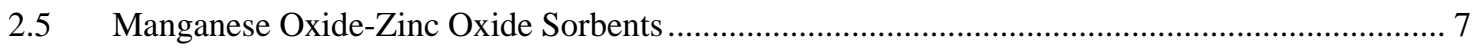

3. BASIC MODELS FOR GAS-SOLID NON-CATALYTIC REACTIONS........................................... 9

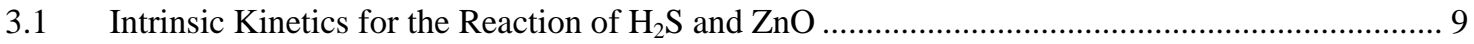

3.2 Modeling of the Heterogeneous Reaction of $\mathrm{H}_{2} \mathrm{~S}$ and Zinc Oxide …....................................... 11

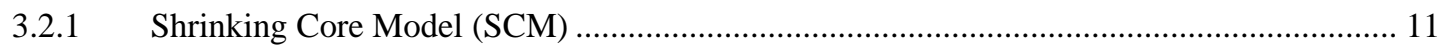

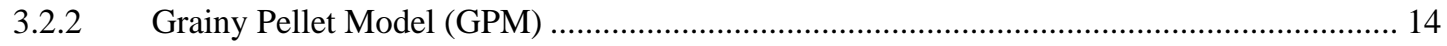

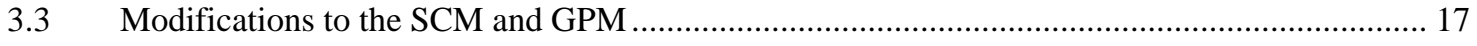

3.3.1 Modifications to Shrinking Core Model ................................................................................. 17

3.3.2 Modifications to Grainy Pellet Model ................................................................................. 18

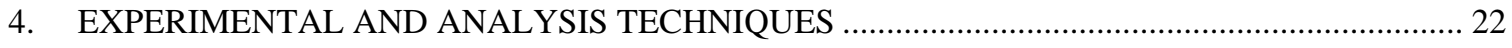

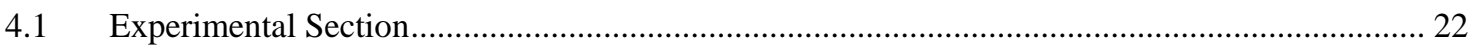




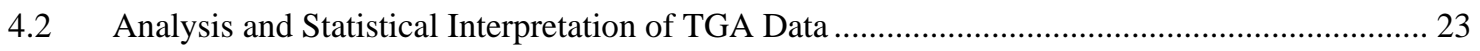

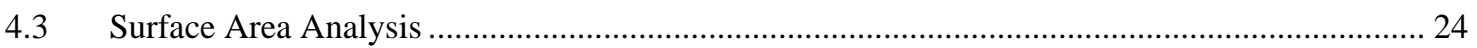

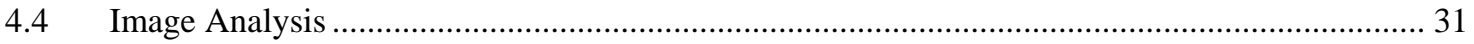

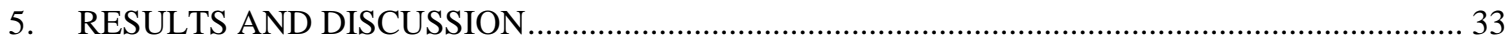

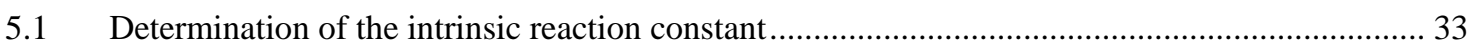

$5.2 \quad$ Predicting conversion using SCM model ................................................................................. 34

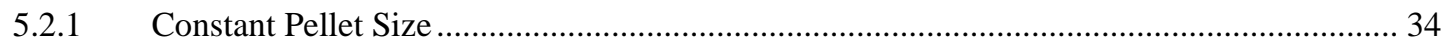

5.2.2 Changing Pellet Size due to Volume Change ........................................................................ 38

5.3 Predicting conversion using Basic GPM model ......................................................................... 39

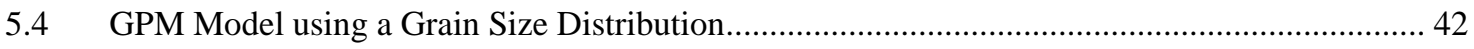

5.4.1 GPM Model using a Bimodal Grain Size ……............................................................ 42

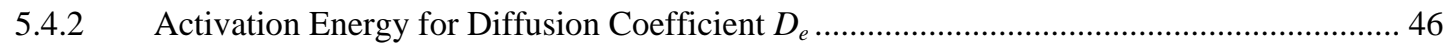

5.4.3 Bimodal GPM for 80 wt \% and 100 wt\% Sorbents .................................................................. 47

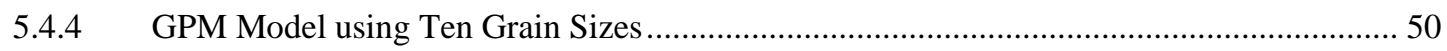

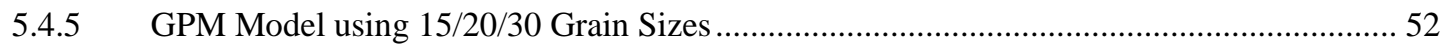

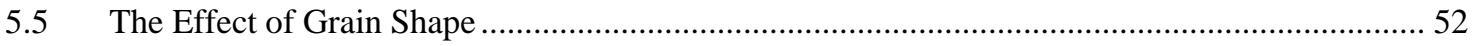

5.5.1 The Effect of Grain Shape on Conversion-Time History ………………….......................... 52

5.5.2 Effect of Different Shaped Grains on Conversion-Time History …………………………..... 54

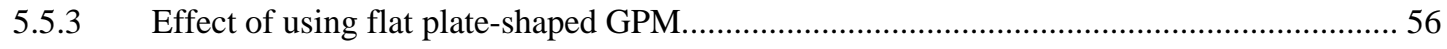

5.5.4 Ten Size Distribution GPM for 80 wt \% Sorbents................................................................ 56

5.6 Images From Scanning Electron Microscope (SEM) …........................................................... 58

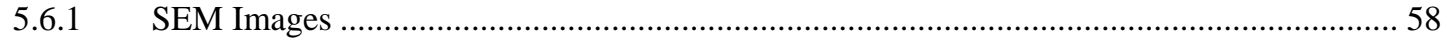

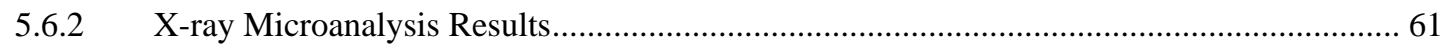

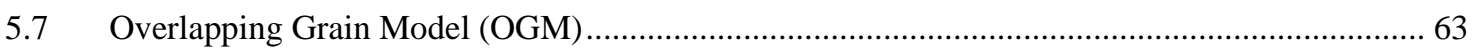

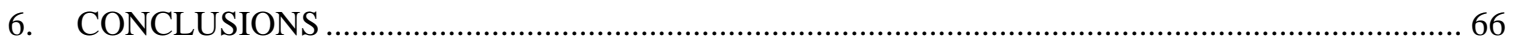

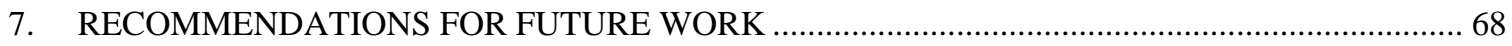

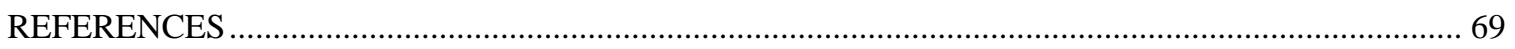

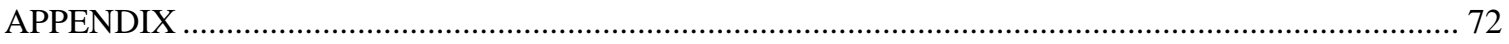




\section{Notation}

A

$A_{\text {ave }}$

$C_{A b}$

$C_{\text {As }}$

$C_{\text {Ac }}$

$C_{B}$

$C_{C}$

$\mathrm{C}_{\mathrm{H}_{2} \mathrm{~S}}$

$D_{e}$

$D_{e m}$

$d_{g}$

E

$E_{A}$

$f\left(C_{j}\right)$

$f(r)$

$k$

$k_{g}$

$L_{\text {ave }}$

$M_{B}$

$M_{\text {singleparticle }}$ frequency factor

average grain surface area of plate-like grain

$\mathrm{cm}^{2} / \mathrm{g}$

concentration of $\mathrm{A}$ in the bulk

$\mathrm{mmol} / \mathrm{cm}^{3}$

concentration of A in the external gas film

surrounding the particle

$\mathrm{mmol} / \mathrm{cm}^{3}$

concentration of A in the surface of unreacted

$\mathrm{mmol} / \mathrm{cm}^{3}$

core of particle

concentration of matter B

$\mathrm{mol} / \mathrm{cm}^{3}$

concentration of matter $\mathrm{C}$

$\mathrm{mol} / \mathrm{cm}^{3}$

concentration of $\mathrm{H}_{2} \mathrm{~S}$ in the reactor

$\mathrm{mmol} / \mathrm{cm}^{3}$

effective diffusivity in the ash layer

$\mathrm{cm}^{2} / \mathrm{min}$

effective diffusion between the grains

$\mathrm{cm}^{2} / \mathrm{min}$

diameter of particle

$\mathrm{cm}$

activation energy for reaction

$\mathrm{kcal} / \mathrm{mol}$

activation energy for diffusion

$\mathrm{kcal} / \mathrm{mol}$

function of concentration of components

function of size distribution

reaction rate constant

$\mathrm{cm} / \mathrm{min}$

external mass transfer coefficient

$\mathrm{cm} / \mathrm{min}$

average grain length of the cylinder

$\mathrm{cm} / \mathrm{g}$

g/mol

molar mass of matter B

mass of single $\mathrm{ZnO}$ particle g 


\begin{tabular}{|c|c|c|}
\hline$M_{\mathrm{ZnO}}$ & molar mass of zinc oxide & $\mathrm{g} / \mathrm{mol}$ \\
\hline$M_{\text {ZnS }}$ & molar mass of zinc sulfide & $\mathrm{g} / \mathrm{mol}$ \\
\hline$m$ & grain shape factor & \\
\hline$N$ & unit number of particle & $1 / g$ \\
\hline$n$ & grain geometric factor & \\
\hline$n_{o}\left(r_{o}\right) d r_{o}$ & $\begin{array}{l}\text { number of grains per unit volume with } \\
\text { radius in the initial size range }\left[r_{o}, r_{o}+d r_{o}\right]\end{array}$ & \\
\hline$P$ & gas pressure & $\mathrm{Pa}$ \\
\hline$R$ & initial (unreacted) particle size & $\mathrm{cm}$ \\
\hline$R_{o}$ & original particle size & $\mathrm{cm}$ \\
\hline$R_{\text {grain,o }}$ & original size of the grains & $\mathrm{cm}$ \\
\hline$R_{\text {pellet }, o}$ & original size of the pellet & $\mathrm{cm}$ \\
\hline$r$ & size of the unreacted core & $\mathrm{cm}$ \\
\hline$r_{A}$ & reaction rate of matter $A$ & $\mathrm{~mol} / \mathrm{m}^{3} \cdot \min$ \\
\hline$r_{B}$ & reaction rate of matter $B$ & $\mathrm{~mol} / \mathrm{m}^{3} \cdot \min$ \\
\hline$r_{o}$ & initial size of grain & $\mathrm{cm}$ \\
\hline$r_{o, \max }$ & maximum initial grain size & $\mathrm{cm}$ \\
\hline$-r_{H_{2} S}$ & disappearance rate of $\mathrm{H}_{2} \mathrm{~S}$ & $\mathrm{~mol} / \mathrm{cm}^{2} \cdot \mathrm{min}$ \\
\hline$-r_{\mathrm{ZnO}}$ & disappearance rate of $\mathrm{ZnO}$ & $\mathrm{mol} / \mathrm{cm}^{2} \cdot \mathrm{min}$ \\
\hline$S_{A}$ & reaction surface area & $\mathrm{cm}^{2} / \mathrm{g}$ \\
\hline$S h$ & Sherwood number & \\
\hline$T$ & reaction temperature & $\mathrm{K}$ \\
\hline$t$ & reaction time & $\min$ \\
\hline$t_{\text {total }}$ & total reaction time & $\min$ \\
\hline
\end{tabular}




\begin{tabular}{|c|c|}
\hline$t_{a s h}$ & $\begin{array}{l}\text { time required to achieve a given conversion } \\
\text { if the process is controlled entirely by } \\
\text { ash-layer diffusion }\end{array}$ \\
\hline$t_{\text {film }}$ & $\begin{array}{l}\text { time required to achieve a given conversion } \\
\text { if the process were controlled only by } \\
\text { external mass transfer }\end{array}$ \\
\hline$t_{\text {reac }}$ & $\begin{array}{l}\text { time that would be needed if chemical } \\
\text { reaction dominated the whole process }\end{array}$ \\
\hline$W_{t}$ & sample mass at time $\mathrm{t}$ \\
\hline$W_{o}$ & original sample mass \\
\hline$W_{X=1}$ & sample mass at complete conversion \\
\hline$X$ & reactant conversion \\
\hline$x_{1}$ & mass fraction of particle 1 \\
\hline$x_{2}$ & mass fraction of particle 2 \\
\hline$Y(t)$ & the lower active reactant grain radius limit \\
\hline$y$ & $y=1-X$ \\
\hline$Z_{y}$ & ratio of molar volume of product to reactant \\
\hline
\end{tabular}

\section{Greek Letters}

$\alpha$

concentration exponent in reaction rate

for component B

$\beta$

concentration exponent in reaction rate

for component $\mathrm{C}$

$\varepsilon$

porosity

$\varphi$

sphericity

$\gamma$

weight fraction of inerts in $\mathrm{ZnO}$ sample

$\rho$

density of pellet

$\mathrm{kg} / \mathrm{m}^{3}$ 


$\begin{array}{lll}\rho_{B} & \text { density of material B } & \mathrm{kg} / \mathrm{m}^{3} \\ \rho_{\text {ZnO }} & \text { density of ZnO } & \mathrm{kg} / \mathrm{m}^{3} \\ \rho_{\text {ZnS }} & \text { density of ZnS } & \mathrm{kg} / \mathrm{m}^{3} \\ \tau_{\text {ash }} & \text { time for complete reaction for ash layer } & \mathrm{s} \\ & \text { diffusion control } & \mathrm{s} \\ \tau_{\text {film }} & \text { time for complete reaction for external } & \mathrm{s} \\ & \text { film diffusion control } & \mathrm{s} \\ \tau_{\text {reac }} & \text { time for complete reaction for surface } & \mathrm{s} \\ \tau_{d_{g 1}} & \text { reaction control } & \\ \tau_{d_{g 2}} & \tau_{a s h} \text { for particle with diameter } d_{g 1} & \\ \phi & \tau_{\text {ash }} \text { for particle with diameter } d_{g 2} & \end{array}$




\section{List of Tables}

Table 3.1: Summary of Intrinsic Rate Parameters for ZnO based Sorbents .................... 10

Table 4.1: Summary of Chemical \& Physical Properties of the Three Sorbents Used. ... 26

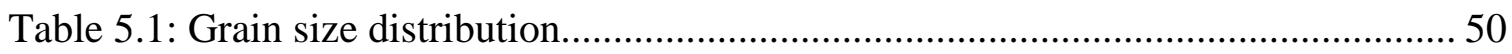

Table 5.2: Mean square error for GPM using 2, 10, 15, 20 and 30 grain sizes ............... 52

Table 5.3: Grain sphericity as a function of diameter................................................ 53 


\section{List of Figures}

Figure 3.1: Schematic representation of the SCM .................................................. 11

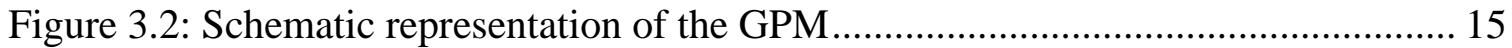

Figure 4.1: Weight Change for Zinc Oxide TGA runs at $60 \%, 1100^{\circ} \mathrm{F}$ and $1 \% \mathrm{H}_{2} \mathrm{~S} \ldots \ldots .27$

Figure 4.2: Expanded View of Figure 4.1 at the time when $\mathrm{H}_{2} \mathrm{~S}$ is injected into the

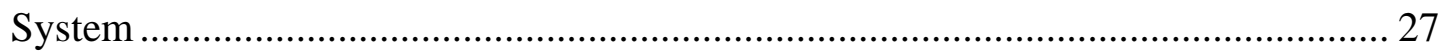

Figure 4.3: Conversion history profile $\left(60 \%, 1100{ }^{\circ} \mathrm{F}, 1 \% \mathrm{H}_{2} \mathrm{~S}\right.$ concentration).............. 28

Figure 4.4: Conversion history profile $\left(60 \%, 900{ }^{\circ} \mathrm{F}, 1 \% \mathrm{H}_{2} \mathrm{~S}\right.$ concentration)................ 28

Figure 4.5: Initial conversion history $\left(60 \%, 1100{ }^{\circ} \mathrm{F}, 1 \% \mathrm{H}_{2} \mathrm{~S}\right.$ concentration)................. 29

Figure 4.6: Initial Conversion history $\left(60 \%, 900{ }^{\circ} \mathrm{F}, 1 \% \mathrm{H}_{2} \mathrm{~S}\right.$ concentration)................. 29

Figure 4.7: Initial Reaction Rate Comparison for Three Sorbents Used assuming the same

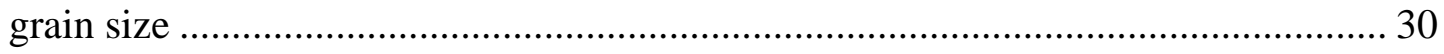

Figure 4.8: Initial Reaction Rate Comparison for Three Sorbents Used $d_{\text {grain }}=0.105 \mu \mathrm{m}$

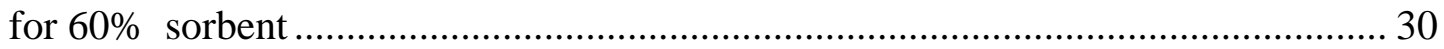

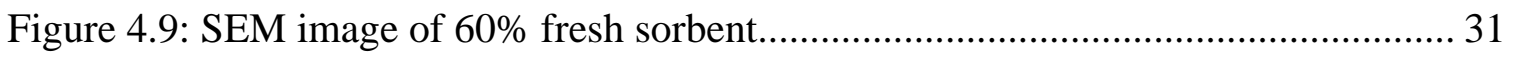

Figure 4.10: Visilog ${ }^{\mathrm{TM}}$ analysis result of SEM image .............................................. 32

Figure 5.1: Comparison of Model Predictions with Experimental Data ......................... 34

Figure 5.2: Experimental results compared with prediction of SCM model $\left(60 \%, 593{ }^{\circ} \mathrm{C}\right.$,

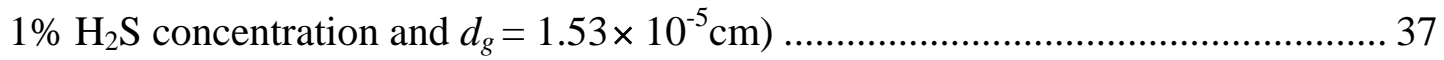

Figure 5.3:Experimental results compared with predictions of SCM with and without $Z_{v}$

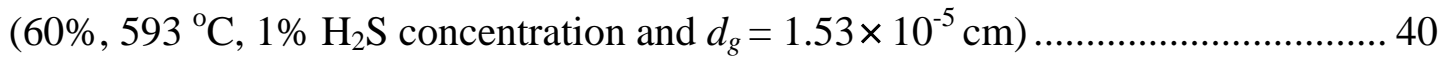

Figure 5.4: Conversion profile for different pellet sizes for $60 \%$ sorbent, $593{ }^{\circ} \mathrm{C}, 1 \% \mathrm{H}_{2} \mathrm{~S}$

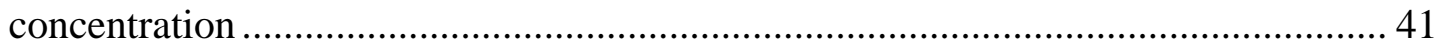


Figure 5.5: Basic GPM compared with experimental data $\left(60 \%, 593{ }^{\circ} \mathrm{C}, 1 \% \mathrm{H}_{2} \mathrm{~S}\right.$ concentration and $\left.d_{g}=1.05 \times 10^{-5} \mathrm{~cm}\right)$ 41

Figure 5.6: Experimental results compared with basic GPM with and without $Z_{v} \quad$ (60\%, $593{ }^{\circ} \mathrm{C}, 1 \% \mathrm{H}_{2} \mathrm{~S}$ concentration and $\left.d_{g}=1.05 \times 10^{-5} \mathrm{~cm}\right)$ 42

Figure 5.7: Experimental results compared with GPM $\left(x_{1}=0.4316 d_{g 1}=7.14 \times 10^{-6}\right.$ $x_{2}=0.5684 d_{g 2}=1.63 \times 10^{-5} \mathrm{~cm}$ ) for $60 \%, 593^{\circ} \mathrm{C}, 1 \% \mathrm{H}_{2} \mathrm{~S}$ concentration 44

Figure 5.8: Experimental results compared with GPM using $\mathrm{Z}_{\mathrm{v}}\left(x_{1}=0.4316\right.$ $\left.d_{g 1}=7.14 \times 10^{-6} x_{2}=0.5684 d_{g 2}=1.63 \times 10^{-5} \mathrm{~cm}\right)$ for $60 \%, 593^{\circ} \mathrm{C}, 1 \% \mathrm{H}_{2} \mathrm{~S}$ concentration 45

Figure 5.9: Experimental results compared with GPM using $\mathrm{Z}_{\mathrm{v}}\left(x_{1}=0.4316\right.$ $\left.d_{g 1}=7.14 \times 10^{-6} x_{2}=0.5684 d_{g 2}=1.63 \times 10^{-5} \mathrm{~cm}\right)$ for $60 \%, 482^{\circ} \mathrm{C}$ and $1 \% \mathrm{H}_{2} \mathrm{~S}$ concentration 45

Figure 5.10: Experimental results compared with GPM using $\mathrm{Z}_{\mathrm{v}}\left(x_{1}=0.57, d_{g 1}=2.8 \times 10^{-5}\right.$ $x_{2}=0.43 d_{g 2}=9.54 \times 10^{-6} \mathrm{~cm}$ ) for $80 \%, 593^{\circ} \mathrm{C}, 1 \% \mathrm{H}_{2} \mathrm{~S}$ concentration 48

Figure 5.11: Experimental results compared with GPM using $Z_{\mathrm{v}}\left(x_{1}=0.57 d_{g 1}=2.8 \times 10^{-5}\right.$ $x_{2}=0.43 d_{g 2}=9.54 \times 10^{-6} \mathrm{~cm}$ ) for $80 \%, 482^{\circ} \mathrm{C}, 1 \% \mathrm{H}_{2} \mathrm{~S}$ concentration 48

Figure 5.12: Experimental results compared with GPM using $Z_{\mathrm{v}}\left(x_{1}=0.4 \quad d_{g 1}=6.02 \times 10^{-5}\right.$ $x_{2}=0.6 d_{g 2}=1.02 \times 10^{-5} \mathrm{~cm}$ ) for $100 \%, 593^{\circ} \mathrm{C}, 1 \% \mathrm{H}_{2} \mathrm{~S}$ concentration 49

Figure 5.13: Experimental results compared with GPM using $Z_{\mathrm{v}}\left(x_{1}=0.4 \quad d_{g 1}=6.02 \times 10^{-5}\right.$ $x_{2}=0.6 d_{g 2}=1.02 \times 10^{-5} \mathrm{~cm}$ ) for $100 \%, 482^{\circ} \mathrm{C}, 1 \% \mathrm{H}_{2} \mathrm{~S}$ concentration $\ldots$

Figure 5.14: Experimental results compared with GPM (ten size distribution) for 60\%, $593^{\circ} \mathrm{C}, 1 \% \mathrm{H}_{2} \mathrm{~S}$ concentration 51 
Figure 5.15: Experimental results compared with GPM (15/20/30 size distribution) for $80 \%, 593^{\circ} \mathrm{C}, 1 \% \mathrm{H}_{2} \mathrm{~S}$ concentration 51

Figure 5.16: Experimental results compared with GPM using sphericity (ten size distribution) for $60 \%, 593^{\circ} \mathrm{C}, 1 \% \mathrm{H}_{2} \mathrm{~S}$ concentration... 55

Figure 5.17: Experimental results compared with ten size GPM (spherical and plate) for $60 \%, 593^{\circ} \mathrm{C}, 1 \% \mathrm{H}_{2} \mathrm{~S}$ concentration 55

Figure 5.18: Experimental results compared with flat-plate shaped using 10 grain sizes GPM for $60 \%, 593^{\circ} \mathrm{C}, 1 \% \mathrm{H}_{2} \mathrm{~S}$ concentration. 57

Figure 5.19: Experimental results compared with GPM (ten grain size distribution) for $80 \%, 593^{\circ} \mathrm{C}, 1 \% \mathrm{H}_{2} \mathrm{~S}$ concentration. 57

Figure 5.20: SEM for fresh 80\% sorbent of 30 time magnification .............................. 58

Figure 5.21: SEM for fresh 80\% sorbent using 8K magnification .............................. 59

Figure 5.22: SEM for fresh 80\% sorbent using 11K magnification .............................. 59

Figure 5.23: SEM for reacted 80\% sorbent using 13K magnification........................... 60

Figure 5.24: SEM for reacted 80\% sorbent using 13K magnification............................ 60

Figure 5.25: Overlay X-ray (Zn \& Al) for fresh 80\% sorbent...................................... 61

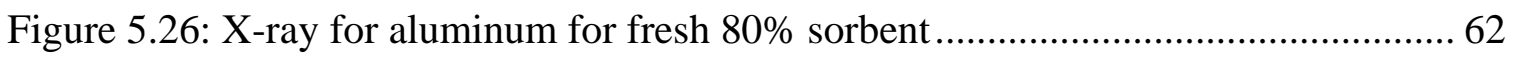

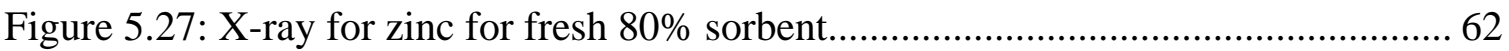




\section{INTRODUCTION}

\subsection{Introduction}

The invention of the incandescent light bulb by Thomas Edison in 1879 created a demand for a cheap, readily available fuel with which to generate large amounts of electric power. Since World War I, coal-fired power plants have accounted for about half of the electricity produced in the U.S. each year. At the same time, coal also brings serious environmental problems: acid rain, waste water, global warming. But compared with other energy sources, such as natural gas and oil, coal has much greater reserves and will stay with us much longer.

One of the major environmental concerns with coal is that it is a fossil fuel and contains some amount of sulfur, which after gasification produces hydrogen sulfide, a toxic gas. Methods to remove hydrogen sulfide are very important not only for coal-fired power plants but in a variety of industrial processes involving the gasification of sulfur containing fuels. The gaseous products from partial combustion and gasification contain hydrogen sulfide that must be removed prior to these gases being discharged to the environment.

Many desulfurization technologies are currently used. However, this research will focus on desulfurizaton using metal oxides to remove hydrogen sulfide from the fuel gas. Direct removal can significantly improve the thermal efficiency of emerging technologies using coal gasification such as integrated gasification combined-cycle (IGCC) and gasifier-molten carbonate fuel cells (MCFC). Hot gas cleanup will also eliminate the costs of heat exchangers to cool down the fuel gas, reheating equipment, and expensive 
wastewater cleanup processes, which are major disadvantages for many low-temperature commercial $\mathrm{H}_{2} \mathrm{~S}$ removal processes.

Various metal oxide sorbents have been considered for high-temperature $\mathrm{H}_{2} \mathrm{~S}$ removal. The potential use of zinc oxide as a high-temperature regenerable sorbent has been investigated [1] and kinetic studies using single pellets of zinc oxide have also been performed [2]. The thermodynamic equilibrium for sulfidation of $\mathrm{ZnO}$ is very favorable, yielding desulfurization down to a few parts per million (ppm) $\mathrm{H}_{2} \mathrm{~S}$. Zinc sulfide can be regenerated providing that sufficiently high temperatures or low oxygen concentrations are used to avoid zinc sulfate formation. In general, zinc oxide is attractive because it combines good sulfidation equilibria, fast kinetics, high sulfur loading and regenerability. However, a major limitation of $\mathrm{ZnO}$-based sorbents for hot gas desulfurization is sorbent loss due to the reduction of zinc oxide to volatile elemental zinc in hot reducing atmospheres.

\subsection{Problem Definition}

The sulfidation of zinc oxide $(\mathrm{ZnO})$ is a non-catalytic, gas-solid reaction characterized by formation of a solid product $(\mathrm{ZnS})$. Thus, there are several transport mechanisms (in the product layer and in the pore space) that must be considered in the analysis and interpretation of experimental data for this reaction. Moreover, if the solid product occupies more space than the solid reactant then the porosity diminishes during the course of the reaction and pore surface area is lost as small pores become plugged with solid product.

Simple mathematical models are usually used to analyze experimental data for the $\mathrm{ZnO}-\mathrm{H}_{2} \mathrm{~S}$ reaction and other sulfidation reactions. For instance, Focht et al. [3] used a 
shrinking core model to interpret their experimental data, while a modified grain model, allowing for swelling effects, was used by Ranade and Harrison [4]. Good agreement is usually obtained between the predictions of the various simple models and experimental reactivity evolution (conversion vs. time) data, but at the expense of letting most of the parameters of the process vary with the operating conditions and in this way compromising the predictive capabilities.

The goal of this project is to investigate the many models that are currently available to describe gas-solid reactions and to determine which of these models best describes the observed (experimental) results and why. An extensive data bank of experimental information on zinc oxide sorbents will be used to compare these models. This data was collected over a period of 1-2 years at the Department of Energy, National Energy Technology Laboratory (DOE/NETL). 


\section{LITERATURE REVIEW}

\section{$2.1 \quad$ Introduction}

Modern power plants using the gasification of coal lead to the formation of sulfurcontaining species, primarily $\mathrm{H}_{2} \mathrm{~S}$, and therefore it is necessary to remove this corrosive/polluting compound prior to the next processing step. Desulfurization of hot coal gas at low temperature (around $300^{\circ} \mathrm{C}$ ) causes loss of most of its sensible heat content, which can be avoided by using regenerable sorbents (metal oxides) to remove the hydrogen sulfide at high temperatures. Hot gas desulfurization offers energy efficiency and avoids costly wastewater treatment. The reaction of hydrogen sulfide with a metal oxide can generally be described as follows:

$$
\text { Oxide }(s)+\mathrm{H}_{2} \mathrm{~S}(g) \rightarrow \text { Sulfide }(s)+\mathrm{H}_{2} \mathrm{O}(g)
$$

Numerous metal oxide sorbents have been investigated for high-temperature, $\mathrm{H}_{2} \mathrm{~S}$ removal. These include pure metal oxides like $\mathrm{ZnO}$, solid mixtures of metal oxides such as zinc ferrite, mixtures of an inert oxide with a solid reactant like zinc titanates, and mixtures of two reactive metal oxides. For example, a mixture might contain a metal oxide such as $\mathrm{MnO}$ that has large desulfurization capacity (expressed as kilogram of sulphur absorbed per kilogram of metal oxide) and another oxide that has high affinity for desulfurization (e.g. $\mathrm{ZnO}$ ). Candidate sorbents are usually evaluated on the basis of the following criteria: the equilibrium concentration of the hydrogen sulfide at the process conditions, the reaction rate during sulfidation and regeneration, the sorbent utilization after different sulfidation/regeneration cycles and the mechanical properties of the solid. Among them, zinc oxide is the most frequently used oxide in desulfurization studies, either as a single oxide or as a mixture with other metal oxides. This is because zinc 
oxide has the highest equilibrium constant for sulfidation and has been singled out as the sorbent of choice for desulfurization of coal gas down to a few parts per million of $\mathrm{H}_{2} \mathrm{~S}$.

Four types of sorbents: pure zinc oxide, zinc ferrite sorbents, zinc titanate-zinc oxide sorbents and manganese oxide- zinc oxide sorbents will be discussed.

\subsection{Zinc Oxide Sorbents}

For high temperatures desulfurization, $\mathrm{ZnO}$ is attractive because the sulfidation of $\mathrm{ZnO}$ leads to low concentration of $\mathrm{H}_{2} \mathrm{~S}$ at the exit of the reactors. The sulfidation of zinc oxide is a noncatalytic gas-solid reaction characterized by formation of a solid product of ZnS. Westmoreland et al. [5] measured the initial reaction rates of zinc oxide $(<170 \mu m)$ in the temperature range of $300^{\circ} \mathrm{C}$ to $800^{\circ} \mathrm{C}$. Efthimiadis and Sotirchos [6] measured conversion-time profiles for zinc oxide sorbents of different sizes $(53-350 \mu m)$. The initial reaction was found to be first order with respect to hydrogen sulfide and zeroth order for zinc oxide.

Gibson and Harrison [2] investigated the reaction between $\mathrm{H}_{2} \mathrm{~S}$ and $\mathrm{ZnO}$ pellet in the temperature range of $375^{\circ} \mathrm{C}$ to $800^{\circ} \mathrm{C}$. They used a grain model to predict the reaction history profile and got a good agreement in the $600-700^{\circ} \mathrm{C}$ temperature range.

Efthimiadis and Sotirchos [6] studied the sulfidation of porous zinc oxide in hydrogen sulfide-nitrogen mixtures at temperature from $300{ }^{\circ} \mathrm{C}$ to $600{ }^{\circ} \mathrm{C}$. The generalized pore model was used and got a good fit with experimental data.

\subsection{Zinc Ferrite Sorbents}

Iron oxide was previously used to desulfurize coke over gases in the ApplebyFrodingham process. However, the high-temperature thermochemistry of the iron oxide - 
hydrogen sulfide reaction prevents desulfurization to the low partial pressures required for fuel cell applications. The thermodynamic properties of zinc oxide are more favorable. The zinc ferrite, formed by combining the single oxides, maintains the favorable thermochemistry of $\mathrm{ZnO}$, reacts rapidly with $\mathrm{H}_{2} \mathrm{~S}$, and is capable of multiple sulfidation-regeneration cycles.

Focht et al. [3] used single cylindrical pellets of $\mathrm{ZnFe}_{2} \mathrm{O}_{4}$ in a thermobalance reactor to measure the conversion-time profile in the temperature range of $500^{\circ} \mathrm{C}$ to $700^{\circ} \mathrm{C}$. They used a shrinking core model to predict the experimental data and got a satisfactory match. Pineda et al. [7] investigated high-temperature desulfurization with zinc ferrite sorbents in a fixed-bed reactor. A grain model with variable properties was used and the predicted and experimental results were in good agreement.

\subsection{Zinc Titanate - Zinc Oxide Sorbents}

Although the thermodynamic equilibrium for sulfidation of $\mathrm{ZnO}$ is quite favorable, yielding desulfurization down to a few parts per million $\mathrm{H}_{2} \mathrm{~S}$, there are major drawbacks of using zinc oxide in that under the reducing atmosphere of the hot coal gas stream, reduction of the oxide may take place at relatively high temperatures $\left(>600^{\circ} \mathrm{C}\right)$ and lead to vaporization of zinc, thus limiting operating temperature. It is found that zinc oxide in association with titanium dioxide is more slowly reduced to volatile zinc than pure zinc oxide. So, when desulfurization operating temperature exceeds $600^{\circ} \mathrm{C}$, zinc titanate is a good choice.

Lew et al. [8] reported the initial reaction rates of zinc titanate sorbents $(90-125 \mu \mathrm{m})$ in the temperature range of $400^{\circ} \mathrm{C}$ to $700^{\circ} \mathrm{C}$. Konttinen et al. [9] measured the conversiontime history of zinc titanate particles (200-308mm) at temperatures in the range 400 - 
$600^{\circ} \mathrm{C}$. In comparison to $\mathrm{ZnO}$, the use of $\mathrm{Zn}$-Ti-O solids allows raising the operating temperature for desulfurization of hot coal gas by as much as $100^{\circ} \mathrm{C}$. The initial sulfidation rate of zinc titanate was approximately half that of pure zinc oxide [8].

The non-catalytic reaction between zinc-titanate and hydrogen sulfide is complex: mass transfer of gaseous reactant from the bulk gas to the pellet exterior surface is followed by diffusion through the pores of the pellet and perhaps through a layer of solid product before the solid reactant is encountered and the surface reaction can occur. However, it is often possible to describe the global rate in terms of relatively simple mathematic models that consider only the most important phenomena and neglect steps that contribute little to the global rate. For example, Jothimurugesan and Harrison [10] used a shrinking core model to analyze the sulfidation reaction between $\mathrm{H}_{2} \mathrm{~S}$ and a singlepellet of zinc titanate sorbent. While, Lew, et al. [11] used a much more complicated overlapping-grain model, to describe the Zn-Ti-O sulfidation. In their overlapping-grain model, the sulfidation rate is proportional to the reactive internal surface area of the porous particles, for which the initial value is obtained by mercury porosimetry.

Konttinen, et al. [9] also studied the hot gas desulfurization with zinc titanate sorbents in a fluidized bed and two different models, the shrinking core model and overlapping grain model, were applied to modeling the reaction history. Both models compared well with the experimental data.

\subsection{Manganese Oxide-Zinc Oxide Sorbents}

Westmoreland, et al. [5] found that the intrinsic sulfidation rate of $\mathrm{MnO}$ was approximately one order of magnitude greater than the rate of $\mathrm{ZnO}$. Compared with zinc oxide, manganese oxide possesses no limitation of operating temperature, while zinc 
oxide can not be used above about $600^{\circ} \mathrm{C}$ because of the formation of zinc vapor. But in terms of the thermodynamic hydrogen sulfide removal efficiency, $\mathrm{MnO}$ is much inferior to $\mathrm{ZnO}$. Thus, manganese oxide has been considered only for applications at very high temperatures $\left(>750^{\circ} \mathrm{C}\right)$ e.g., coal-gas desulfurization.

The sorbents combining two chemical compounds have lead to enhanced sorbent performance during sulfidation. The second phase can provide dispersion of the active sorbent phase or form compounds with it that have lower reducibility than the uncombined active oxides. Thus, the mixed metal oxide sorbents can improve sorbent regenerability and structural stability in cyclic operation and, increase the strength and attrition resistance of the sorbents.

Li et al. [12] studied hot gas clean up using a ZnO-MnO desulfurizer and they used an equivalent grain model to describe the reaction history.

For this project, the focus is on high temperature desulfurization with pure zinc oxide as a sorbent. Modeling of the system will investigate the variations of SCM or GPM to predict the conversion-time profile. 


\section{BASIC MODELS FOR GAS-SOLID NON-CATALYTIC REACTIONS}

\subsection{Intrinsic Kinetics for the Reaction of $\mathrm{H}_{2} \mathrm{~S}$ and $\mathrm{ZnO}$}

The basic reaction between hydrogen sulfide and zinc oxide is given by:

$$
\mathrm{ZnO}(s)+\mathrm{H}_{2} \mathrm{~S}(g) \rightarrow \mathrm{ZnS}(s)+\mathrm{H}_{2} \mathrm{O}(g)
$$

Generally, the intrinsic rate of reaction is obtained from the initial rate data using a TGA (Thermo-Gravimetric Analyzer) or equivalent apparatus. It is well known that the intrinsic kinetics follows the form:

$$
-r_{B}=[k(T)]\left[f\left(C_{B}, C_{C}, \ldots\right)\right]
$$

That is, the rate of disappearance of component $B$, depends on temperature and composition. The reaction rate constant $k$ is not truly a constant, but is nearly independent of the concentrations of the species involved in the reaction. It is almost always strongly dependent on temperature. For this project, we assume the reaction rate constant, $k$, obeys the following Arrhenius equation:

$$
k(T)=A e^{-E / R T}
$$

where $A$ is a pre-exponential factor or frequency factor; $E$ is the activation energy; $R$ is the gas constant and $T$ is the absolute temperature.

The dependence of the reaction rate $-r_{B}$ on the concentrations of the species present, $f\left(C_{j}\right)$, is almost without exception determined by experimental observation. For elementary reaction mechanism this dependence is the product of concentrations of the individual reaction species, each raised to some power, e.g.,

$$
-r_{B}=k C_{B}^{\alpha} C_{C}^{\beta}
$$


So, for this case, the rate of disappearance of $\mathrm{H}_{2} \mathrm{~S}$ or $\mathrm{ZnO}$ can be expressed as:

$$
-r_{\mathrm{H}_{2} \mathrm{~S}}=-r_{\mathrm{Zn} 0}=k C_{\mathrm{H}_{2} \mathrm{~S}}^{\alpha} C_{\mathrm{ZnO}}^{\beta}
$$

A summary of previously determined intrinsic rate parameters for $\mathrm{ZnO}$ based sorbents is given in Table 3.1 below.

Table 3.1: Summary of Intrinsic Rate Parameters for ZnO based Sorbents

\begin{tabular}{|c|c|c|c|c|c|}
\hline $\begin{array}{c}\mathrm{H}_{2} \mathrm{~S} \\
\text { concentration } \\
\text { dependence, } \\
\alpha\end{array}$ & $\begin{array}{c}\text { ZnO } \\
\text { concentration } \\
\text { dependence, } \\
\beta\end{array}$ & $\begin{array}{c}\text { Exponential } \\
\text { constant, } A \\
(\mathrm{~cm} / \mathrm{s})\end{array}$ & $\begin{array}{c}\text { Activation } \\
\text { Energy, } E \\
(\mathrm{kcal} / \mathrm{mol})\end{array}$ & $\begin{array}{c}\text { Experimental } \\
\text { Temperature } \\
\text { Range }\left({ }^{\circ} \mathrm{C}\right)\end{array}$ & Reference \\
\hline 1 & 0 & 0.046 & 7.59 & $540-600$ & $7^{2}$ \\
\hline 1 & 0 & 1.31 & $\begin{array}{c}10.3 \mathrm{ZnO} \\
0.3 \mathrm{ZnTi}\end{array}$ & $400-800$ & $8^{1}$ \\
\hline 1 & 0 & $0.0014-$ & 5.98 & $400-600$ & $9^{3}$ \\
\hline 1 & 0 & 0.0025 & 7.24 & $400-800$ & 13 \\
\hline
\end{tabular}

Note: ${ }^{1}$ zinc oxide and zinc titanates were studied, ${ }^{2}$ results for zinc-ferrite, ${ }^{3}$ results for zinc titanates

From the table above, all the researchers have assumed or determined that the dependence of the reaction rate on hydrogen sulfide concentration is first order, that is, $\alpha=1$, and the dependence of the reaction rate on the zinc oxide concentration is zero order, i.e., $\beta=0$. So the intrinsic kinetics of the reaction between hydrogen sulfide and zinc oxide are:

$$
-r_{H_{2} S}=-r_{Z n O}=k C_{H_{2} S}
$$




\subsection{Modeling of the Heterogeneous Reaction of $\mathrm{H}_{2} \mathrm{~S}$ and Zinc Oxide}

The sulfidation of $\mathrm{ZnO}$ is a non-catalytic gas-solid reaction. For this type of reaction, there are two common models to describe the reaction phenomenon; the sharp interface model (shrinking core model or SCM) [14] and the grainy pellet model (GPM) [14]. Each of these models is described below:

\subsubsection{Shrinking Core Model (SCM)}

In the shrinking core model, which is restricted to non-porous solids, the reaction is assumed to occur at a sharp interface between the exhausted outer shell and the unreacted core of the solid. The unreacted core shrinks in size as the reaction proceeds. A schematic representation of the model is shown in Figure 3.1.

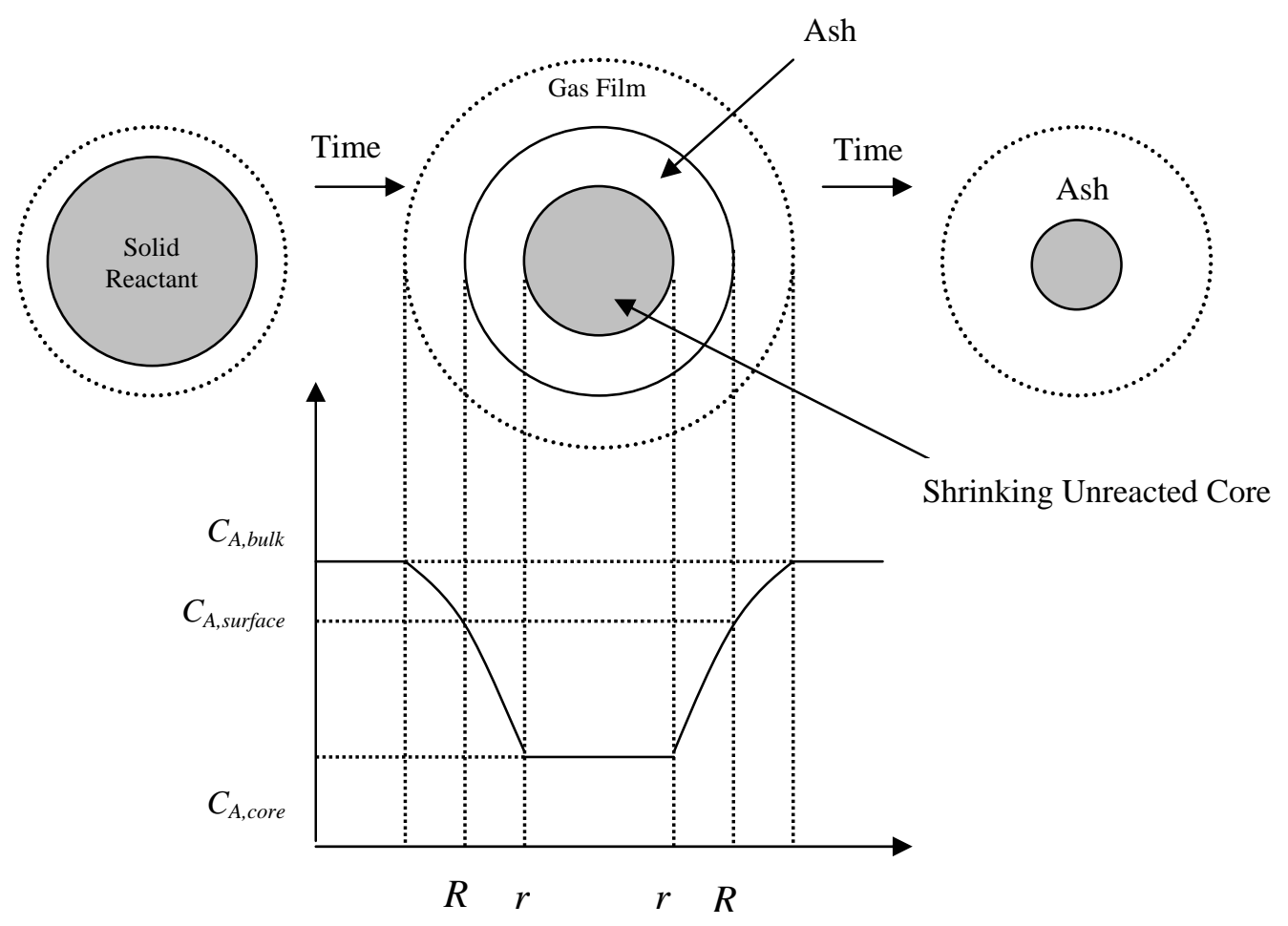

Figure 3.1: Schematic representation of the SCM 
For this model, the following assumptions are used to derive the equations describing the conversion-time history of the particles.

$>$ Restricted to non-porous solids

$>$ Isothermal

$>$ Constant pellet size

$>$ Equimolar counter diffusion of gases through outer gas film and product layer

$>$ Pseudo-steady state approximation, which means that the reaction interface can be assumed to remain stationary at any time, while a steady-state diffusion flux is calculated to find the concentration profile.

$>1^{\text {st }}$ order irreversible reaction

The various steps involved in modeling the overall reaction, and the corresponding rates, are as follows.

1. Diffusion through the external gas film surrounding the particle (external mass transfer resistance) can be expressed as:

$$
-r_{A}=4 \pi R^{2} k_{g}\left(C_{A b}-C_{A s}\right)
$$

2. Diffusion through the product layer (ash layer) based on the assumption of equimolar counter diffusion can be expressed as:

$$
-r_{A}=\frac{4 \pi R r D_{e}}{R-r}\left(C_{A s}-C_{A c}\right)
$$

3. Chemical reaction at the interface can be expressed as:

$$
-r_{A}=4 \pi r^{2} k C_{A c}
$$


The rate of reaction at a given time $t$ (when the position of the reaction interface is at $r$ ) is given by combining Equations (6), (7), (8) and eliminating the interface concentrations:

$$
-r_{A}=\left(\frac{1}{4 \pi R^{2} k_{g}}+\frac{R-r}{4 \pi R r D_{e}}+\frac{1}{4 \pi r^{2} k}\right)^{-1} C_{A b}
$$

Here, the definition of the reaction rate is:

$$
-r_{A}=-\frac{d}{d t}\left(\frac{4}{3} \pi r^{3} \frac{\rho_{B}}{M_{B}}\right)
$$

In gas-solid reactions, the quantity of interest is the fractional conversion $(X)$ of the solid reactant. For a spherical solid, this is related to $r$ by:

$$
X=1-\left(\frac{r}{R}\right)^{3}
$$

Combining Equations (9), (10) and (11) and integrating, the following relationship between conversion and time is obtained:

$$
\frac{R}{3 k_{g}} X+\frac{R^{2}}{6 D_{e}}\left[1-3(1-X)^{2 / 3}+2(1-X)\right]+\frac{R}{k}\left[1-(1-X)^{1 / 3}\right]=\frac{C_{A b} M_{B}}{\rho_{B}} t
$$

Rearranging Equation (12) gives:

$$
t=t_{\text {total }}=t_{\text {film }}+t_{\text {ash }}+t_{\text {reac }}
$$

where, $\quad t_{\text {film }}=\tau_{\text {film }} X ; \tau_{\text {film }}=\frac{\rho_{B} R}{3 k_{g} C_{A b} M_{B}}$

$$
\begin{aligned}
& t_{\text {ash }}=\tau_{\text {ash }}\left[1-3(1-X)^{2 / 3}+2(1-X)\right] ; \tau_{\text {ash }}=\frac{\rho_{B} R^{2}}{6 D_{e} C_{A b} M_{B}} \\
& t_{\text {reac }}=\tau_{\text {reac }}\left[1-(1-X)^{1 / 3}\right] ; \tau_{\text {reac }}=\frac{\rho_{B} R}{k C_{A b} M_{B}}
\end{aligned}
$$


The physical meaning of these quantities is as follows: $t_{\text {film }}$ is the time required to achieve a given conversion if the process were controlled only by external mass transfer; $t_{a s h}$ is the time required to achieve a given conversion if the process are controlled entirely by ash-layer diffusion; $t_{\text {reac }}$ is the time that would be needed if chemical reaction dominated the whole process. While, $\tau_{\text {film }}, \tau_{\text {ash }}$ and $\tau_{\text {reac }}$ are the times for complete reaction $(X=1)$ for each of the three above controlling regimes.

Thus, the following $X$ - $t$ relationships, for different controlling regimes, are found:

1. gas-film diffusion control, $t \propto X$

2. ash diffusion control, $t \propto\left[1-3(1-X)^{2 / 3}+2(1-X)\right]$

3. chemical reaction control, $t \propto\left[1-(1-X)^{1 / 3}\right]$

\subsubsection{Grainy Pellet Model (GPM)}

For the grainy pellet model, the solid reactant is visualized as being composed of a large number of highly dense, spherical grains, each of which reacts individually according to the shrinking core model. Reactant gas undergoes mass transfer from the bulk gas stream to the pellet surface. From the surface, the gas diffuses between the grains, then through a solid product layer associated with each grain until reaction occurs at the unreacted core present in each grain. Thus, four resistances are included, namely, bulk diffusion resistance, macropores diffusion resistance, ash layer diffusion resistance and chemical reaction resistance. For this model, we can first consider the rate of reaction of individual grains and then incorporate it in the mass balance of solid reactant in the macropores of the pellets. A schematic diagram of the model is shown in Figure 3.2. 
The following assumptions are used in the development of this model:

$>$ Pellets are assumed to be comprised of a number of small spherical impervious grains.

$>$ The spaces between the grains are the macropores for intra-pellet diffusion of gas.

Each grain follows a SCM and a product or ash layer is assumed to form.

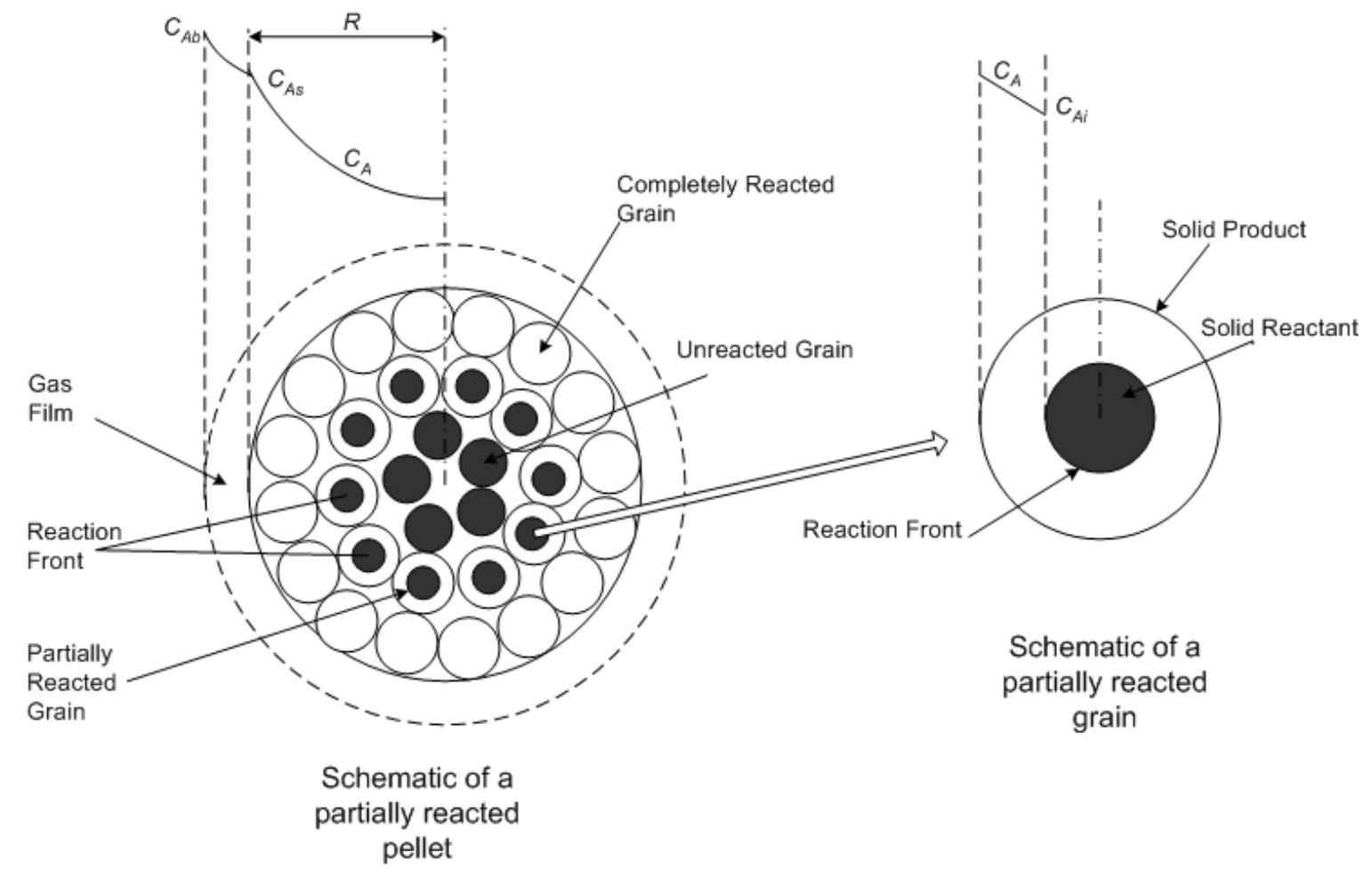

Figure 3.2: Schematic representation of the GPM

From the above figure, it can be seen that each grain acts like an SCM particle, but different extents of reaction are seen for the grains depending on their location within the pellet.

A general dimensionless representation of the grain model has been proposed by Szekely and Sohn [14], which allows for spherical and flat-plate like pellets made up of spherical or flat-plate like grains. This model is based on negligible diffusional gradients 
within the grains. Szekely and Sohn [14] also defined a generalized Thiele modulus given below:

$$
\phi=R_{\text {pellet }, o}\left[\frac{3(1-\varepsilon) k}{D_{\text {em }} R_{\text {grain }, o}}\right]^{1 / 2}
$$

Here, $R_{\text {pellet,o }}$ is the original size of the pellet, $R_{\text {grain,o }}$ is the original size of the grains, $D_{e m}$ is the effective diffusion between the grains, and $\varepsilon$ is the porosity of the pellet.

They have noted two patterns of asymptotic behavior for the system.

1. $\phi \rightarrow 0$ (kinetic control)

The concentration within the pellets is uniform and all the grains are exposed to the same gas concentration. For no external (pellet) resistance we have:

$$
\hat{t}=1-(1-X)^{1 / 3}
$$

Here, $\hat{t}$ is a dimensionless time defined as:

$$
\hat{t}=\frac{C_{A b} M_{B} k t}{\rho_{B} R_{\text {grain }, o}}
$$

2. $\phi \rightarrow \infty$ (diffusion control)

A sharp demarcation can be observed between the reacted and unreacted portions of the pellet and the behavior is similar to that of the SCM. The $X$ vs. $\hat{t}$ relationship now is:

$$
\hat{t}=\frac{\phi^{2}}{18}\left[1-3(1-X)^{2 / 3}+2(1-X)\right]
$$


3. For intermediate values of $\phi$, Sohn and Szekely proposed the following approximate solution for conversions for a spherical pellet comprising spherical grains:

$$
\hat{t}=1-(1-X)^{1 / 3}+\frac{\phi^{2}}{18}\left[1-3(1-X)^{2 / 3}+2(1-X)+\frac{2 X}{S h}\right]
$$

Here, Sh is Sherwood number.

\subsection{Modifications to the SCM and GPM}

In general, the basic forms of the SCM and GPM models have not been able to predict correctly the observed conversion-time data for the zinc oxide sulfidation reaction because the first virtually ignores solid structural properties while the second considers solid properties but requires that they remain invariant during the course of reaction. Instead, variations of these models have been suggested to account for the observed behavior in zinc oxide sulfidation and other gas-solid non-catalytic reactions. The most important variations or extensions to these models are: the effect of conversion on the physical properties of the sorbent due to the difference in molecular volume of zinc oxide and zinc sulfide, Shen and Smith [15]; the non-homogeneous physical structure of the sorbent, including overlapping grains, Stirchos and Yu [16] and size distribution of grains, Bartlett et al. [17].

\subsubsection{Modifications to Shrinking Core Model}

When the solid product layer $(\mathrm{ZnS})$ does not have the same volume as the solid reactant $(\mathrm{ZnO})$ consumed, a change in particle size will occur. This can be modeled by introducing an additional constant, $Z_{v}[15]$, into the SCM.

$$
Z_{v}=\frac{\text { Volume of product formed }}{\text { Volume of reactant consumed }}=\frac{\rho_{\mathrm{Zno}} / M_{\mathrm{ZnO}}}{\rho_{\mathrm{ZnS}} / M_{\mathrm{ZnS}}}
$$


From the stoichiometry of the reaction,

$$
\frac{\rho_{\mathrm{ZnO}}\left(R_{o}^{3}-r^{3}\right)}{M_{\mathrm{ZnO}}}=\frac{\rho_{\mathrm{ZnS}}\left(R^{3}-r^{3}\right)}{M_{\mathrm{ZnS}}}
$$

where $R_{o}$ is the initial radius of the pellet. Rearranging Equation (22) and inserting $Z_{v}$ :

$$
R^{3}=R_{o}^{3}\left[Z_{v}+\left(1-Z_{v}\right)\left(\frac{r}{R_{o}}\right)^{3}\right]
$$

Substituting Equation (23) into Equation (9), we get,

$$
-r_{A}=\left[\frac{1}{4 \pi\left[R_{o}^{3} Z_{v}+\left(1-Z_{v}\right) r^{3}\right]^{2 / 3} k_{g}}+\frac{\left[R_{o}^{3} Z_{v}+\left(1-Z_{v}\right) r^{3}\right]^{1 / 3}-r}{4 \pi\left[R_{o}^{3} Z_{v}+\left(1-Z_{v}\right) r^{3}\right]^{1 / 3} r D_{e}}+\frac{1}{4 \pi r^{2} k}\right]^{-1} \cdot C_{A b}
$$

Recalling that for spherical pellets, $X=1-\left(\frac{r}{R_{o}}\right)^{3}$, the equation above becomes:

$$
-r_{A}=4 \pi R_{o}^{2}\left[\frac{1}{\left[Z_{v}+\left(1-Z_{v}\right)(1-X)\right]^{2 / 3} k_{g}}+\frac{R_{o}\left[\left[Z_{v}+\left(1-Z_{v}\right)(1-X)\right]^{1 / 3}-(1-X)^{1 / 3}\right]}{\left[Z_{v}+\left(1-Z_{v}\right)(1-X)\right]^{1 / 3}(1-X)^{1 / 3} D_{e}}+\frac{1}{(1-X)^{2 / 3} k}\right]^{-1} \cdot C_{A b}
$$

\subsubsection{Modifications to Grainy Pellet Model}

\section{Swelling}

In the GPM, it was assumed the grains do not change size during the reaction process. Actually, just as in the SCM, a parameter, $Z_{v}$, can be introduced to account for the swelling or shrinking of the grains.

\section{Grain Size Distribution}

For most practical situations, the initial grain size is not uniform. The GPM can be modified to consider a distribution of grain sizes. For example, it may be assumed that the grains are composed of $n$ different sizes: $d g_{1}, d g_{2} \ldots d g_{n}$. From the known values, 
i.e., the particle density and the area of the reaction surface, we can determine the mass fraction and dimensions of each grain sizes. Thus, for each grain size, we can get the conversion history profile according to the basic GPM. The total conversion of the pellet can be calculated as follows:

$$
X=1-x_{1}\left(1-X_{1}\right)-x_{2}\left(1-X_{2}\right)-\cdots-x_{n}\left(1-X_{n}\right)
$$

Where, $x_{i}$ is the mass fraction of grain size $d g_{i}$ and $X_{i}$ is the corresponding conversion of grain size $d g_{i}$.

\section{$>$ Overlapping Grain Model (OGM)}

One weakness of the grain model lies in the assumption that the solid is composed of non-overlapping grains, where the individual grains grow independently with nonoverlapping of the product layer. Lindner and Simonsson [18] represented the initial solid structure as an aggregate of overlapping spheres in an initial stage of sintering. Sotirchos and $\mathrm{Yu}$ [16] further refined this overlapping grain model by representing the solid as an assemblage of grains randomly placed in space with possible overlapping.

The overlapping grain model is more flexible and powerful than the basic grain model in that it can predict various behavior, e.g., a maximum in the rate-conversion profile. The grain model predicts a monotonically decreasing reaction surface area with conversion because the reacting surface of each grain is receding. But for some systems there is anomaly of a monotonically increasing pore surface area even when the porosity reduces to zero [14].

In this model, the porosity $\varepsilon_{r}$ is defined as: 


$$
\varepsilon_{r}=\exp \left[-n \int_{Y(t)}^{r_{o, \max }} r^{m} n_{o}\left(r_{o}\right) d r_{o}\right]
$$

where, $n$ is the grain geometric factor (sphere $=4 \pi / 3$; cylinder $=\pi / L_{\text {ave }}$; plate $=2 A_{\text {ave }}$ ); $m$ is the grain shape factor (sphere=3; cylinder=2; plate=1); $L_{\text {ave }}$ and $A_{\text {ave }}$ are the average grain length of the cylinder and the average grain surface area of plate-like grains, respectively; $r$ is the grain radius; $r_{o}$ is the initial grain radius; $r_{o, \max }$ is the initial maximum grain radius; $n_{o}\left(r_{o}\right) d r_{o}$ is the number of grains per unit volume with radius in the initial size range $\left[r_{o}, r_{o}+d r_{o}\right]$ and $Y(t)$ is the lower active reactant grain radius limit.

The porosity of the solid (reactant product) is found from

$$
\varepsilon_{p}=\varepsilon_{o}-\left(Z_{v}-1\right)\left(\varepsilon_{r}-\varepsilon_{o}\right)
$$

where $\varepsilon_{0}$ is the initial porosity of solids.

And, the fractional conversion is calculated as

$$
X=\frac{\varepsilon_{o}-\varepsilon_{p}}{\left(Z_{v}-1\right)\left(1-\varepsilon_{o}\right)}
$$

For this model, the initial grain size distribution can be determined by SEM, while the pore size distribution and surface area can be obtained by standard measurements.

\section{$>$ Random Pore Model (RPM)}

The random pore model developed by Bathia and Perlmutter [19] is another useful tool to analyze gas-solid reactions. It allows for arbitrary pore size distributions in the reacting solid. This model utilizes a pore structure parameter to characterize solid reactivity, and the analysis relates this parameter to $m$, the grain shape factor. 
They also defined the following:

$$
\begin{aligned}
& V_{E}=\pi \int_{0}^{\infty} r^{2} f(r) d r \\
& S_{E}=2 \pi \int_{0}^{\infty} r f(r) d r \\
& L_{E}=\int_{0}^{\infty} f(r) d r
\end{aligned}
$$

as the total enclosed volume, total surface area and total length of the non-overlapped cylindrical system respectively. Here, $f(r)$ is particle size distribution

By using the characteristic parameters of a random pore size distribution, pore volume, surface area and length, this model avoids the assumption of an idealized structure having uniformly sized cylindrical pores. This model also can predict the reaction surface area at any given conversion as a function of an initial pore structure parameter. 


\section{EXPERIMENTAL AND ANALYSIS TECHNIQUES}

\subsection{Experimental Section}

The conversion-time histories of three sorbents (60\%, $80 \%$ and $100 \%$ mass fraction of $\mathrm{ZnO}$ ) were found using a TA Instruments model TGA 2950 Thermogravimetric Analyzer with an EGA (Evolved Gas Analysis) Furnace. These sorbents were supplied by Intercat (Savannah, GA) and contained up to $40 \mathrm{wt} \%$ binder. The size range of particles for each sorbent was quite board with average diameters in the range of 60$80 \mu \mathrm{m}$. A sample weight of approximately $3 \mathrm{mg}$ of sorbent was used for all tests with the gas stream comprising of $140 \mathrm{cc} / \mathrm{min}$ of nitrogen containing from 0.5 to $2 \mathrm{~mol} \% \mathrm{H}_{2} \mathrm{~S}$. The experiment was operated over the range of $482^{\circ} \mathrm{C}\left(900^{\circ} \mathrm{F}\right)$ to $593{ }^{\circ} \mathrm{C}\left(1100^{\circ} \mathrm{F}\right)$ at 1 atmosphere.

Initial screening experiments were carried out to estimate the effects of gas flow on the observed kinetics. For the sample weight and gas flow given above, the effect of external mass transfer on the initial observed reaction rates was found to be negligible. Initial reaction rate data for three sorbent samples were correlated based on the surface area of zinc oxide available for reaction

Typical experimental data for zinc oxide are given in Figure 4.1. An expanded plot of the initial rate of the sample is shown in Figure 4.2. For the run illustrated in these figures, stabilization of the sample was achieved at a time of about 200 minutes after start-up. At some time after this point, $\mathrm{H}_{2} \mathrm{~S}$ is added and mixed with the incoming nitrogen and the sulfidation reaction is started. From studying Figure 4.2, this time occurs somewhere close to 205 minutes into the experiment. The change in weight of the sample is evident at this time and the initial rate of change of the sample weight is taken to be the 
slope of the curve at this point. In order to obtain a representative slope, the average slope over a period of about one minute is taken. From Figure 4.2, it is clear that the rate of change of this slope is slow enough that averaging the slope over a period of a minute or so will not bias the value. This process for estimating the initial slope was adopted for all experiments.

\subsection{Analysis and Statistical Interpretation of TGA Data}

By performing sulfidation of zinc oxide using thermogravimetric analysis (TGA), a collection of experimental data was obtained, which gave the change in sample weight with time. We can transform the weight vs. time data into conversion vs. time data. The basic definition of conversion, $X$, is given as follows:

$$
X=\frac{W_{t}-W_{o}}{W_{X=1}-W_{o}}
$$

where $W$ refers to the weight of the sample and the subscript refers to time. The initial weight of the sample is known and the weight of sample at complete conversion $X=1$, can either be calculated from the initial weight of the sample based on the stoichiometry or can be inferred from the weight of the sample in the TGA at the end of reaction providing that all the reactant has been consumed. Here we use the stoichiometric relationship to calculate the final sample weight, because there is no guarantee that complete conversion will be achieved. The weight of the sample at any time, $t$, is measured by the microbalance of the TGA. For the zinc oxide-zinc sulfide system, the calculations are as follows:

$$
W_{o}=\left(W_{\text {ZnO }}\right)_{o}+W_{\text {inert }}
$$




$$
\begin{aligned}
& W_{X=1}=\left(W_{Z n O}\right)_{o}\left[\frac{M_{Z n S}}{M_{Z n O}}\right]+W_{\text {inert }}=\left(W_{Z n O}\right)_{o}\left(\frac{97.4}{81.4}\right)+W_{\text {inert }}=1.1966\left(W_{Z n O}\right)_{o}+W_{\text {inert }} \\
& W_{X=1}-W_{o}=1.196\left(W_{Z n O}\right)_{o}+W_{\text {inert }}-\left(W_{Z n O}\right)_{o}-W_{\text {inert }}=0.1966\left(W_{Z n O}\right)_{o} \\
& \left(W_{Z n O}\right)_{o}=(1-\gamma) W_{o} \\
& W_{X=1}-W_{o}=0.1966(1-\gamma) W_{o}
\end{aligned}
$$

where, $\gamma$ is the weight fraction of inerts in $\mathrm{ZnO}$ sample.

Then, Equation (33) becomes:

$$
X=\frac{W_{t}-W_{o}}{0.1966(1-\gamma) W_{o}}
$$

The reaction profile (60\% sorbent) for a $1 \% \mathrm{H}_{2} \mathrm{~S}$ concentration in nitrogen at $593^{\circ} \mathrm{C}$ and $482^{\circ} \mathrm{C}$ are shown in Figure 4.3 and Figure 4.4 respectively. Figure 4.5 and 4.6 show the initial conversion histories.

\subsection{Surface Area Analysis}

BET surface area analyses using nitrogen were carried out using a Quantachrome Autosorb-6 Automated Gas Sorption System (Auantachrome Instrument, Boynton Beach, Florida). For chemisorption analysis, a Micrometritics ASAP 2010C Chemisorption Analyzer (Micrometritics Instrument Company, Norcross, GA) was employed using both ammonia and t-butyl amine.

For the $60 \%$ and $80 \%$ (mass fraction of $\mathrm{ZnO}$ ) sorbents, the BET surface areas reflect both the active $(\mathrm{ZnO})$ and inactive (binder) surface area. This fact is clearly shown in Table 4.1 where the BET surface area for the $100 \mathrm{wt} \%$, $80 \mathrm{wt} \%$ and $60 \mathrm{wt} \%$ are given as 5.91, 44.18 and $89.69 \mathrm{~m}^{2} / \mathrm{g}$, respectively. The large increase in surface area is clearly a 
function of the increasing content of alumina binder that overshadows the decreasing active surface area of the zinc oxide.

From the above, it was assumed that the surface area of the sorbents has the following linear relationship:

$$
S_{A}=(1-\gamma) S_{A Z n O}+\gamma S_{\text {ABinder }}
$$

Substituting the BET surface areas into Equation (35), we get the best-fit regression value of $S_{A B i n d e r}=211 \mathrm{~m}^{2} / \mathrm{g}$, which falls in the range of specific surface area for alumina supports, $80-350 \mathrm{~m}^{2} / \mathrm{g}$ [20]. We further assume that the zinc oxide is distributed in the form of small spherical grains within the alumina support, and the average grain size for all sorbent compositions is the same. Then the initial active surface area of the sorbent can be expressed as:

$$
\left.S_{A}\right|_{t=0}=\frac{6(1-\gamma)}{\rho_{Z n O} \cdot d_{g}}
$$

Here, $\rho_{Z n O}$ is the density of pure zinc oxide $\left(\rho_{\mathrm{ZnO}}=5.6 \mathrm{~g} / \mathrm{cm}^{3}\right)$.

For the $100 \mathrm{wt} \%$ sorbent $(\gamma=0)$, which has a surface area of $7.01 \times 10^{4}\left(\mathrm{~cm}^{2} / \mathrm{g}\right)$, the average grain diameter is obtained as $d_{g}=0.153 \mu \mathrm{m}$. The active surface area for $60 \mathrm{wt} \%$ and $80 \mathrm{wt} \%$ sorbents were estimated from Equation (36) using this average grain diameter.

Experiments were performed to determine the initial rate of reaction for each of the sorbents under various temperatures and $\mathrm{H}_{2} \mathrm{~S}$ concentrations. The initial active surface areas obtained from Equation (36) were used with the initial reaction rate data from these experiments and a comparison of all three sorbents is shown Figure 4.7. It is clear that the 
rates for the $100 \mathrm{wt} \%$ and $80 \mathrm{wt} \%$ sorbent agree well but that the results for $60 \mathrm{wt} \%$ sorbent are significantly higher. A better fit was obtained by using the effective average grain dimensions as a fitting parameter, which gives, $\bar{d}_{g}=0.105 \mu \mathrm{m}$ for the $60 \%$ sorbents, which is shown in Figure 4.8.

Table 4.1: Summary of Chemical \& Physical Properties of the Three Sorbents Used.

\begin{tabular}{|c|c|c|c|}
\hline Nominal Composition & 100\% Fresh Sorbent & 80\% Fresh Sorbent & 60\% Fresh Sorbent \\
\hline $\begin{array}{r}\text { ZnO Content from ICP } \\
(\mathrm{wt} \%)\end{array}$ & 99.9 & 83.1 & 59.7 \\
\hline $\begin{array}{c}\left.\text { Bulk Density } / \mathrm{cm}^{3}\right) \\
\text { Porosity }\end{array}$ & 0.9174 & 1.2302 & 1.0667 \\
\hline \begin{tabular}{c} 
BET Surface Area $\left(\mathrm{m}^{2} / \mathrm{g}\right)$ \\
\hline $\begin{array}{c}\text { Chemisorb SA } \\
\left(\mathrm{cm}^{3}-\text { ammonia/g) }\right.\end{array}$
\end{tabular} & $73.0 \%$ & $74.4 \%$ & $73.2 \%$ \\
\hline \begin{tabular}{c} 
Total Pore Area $\left(\mathrm{m}^{2} / \mathrm{g}\right)$ \\
\hline
\end{tabular} & 7.007 & 44.18 & 89.69 \\
\hline
\end{tabular}




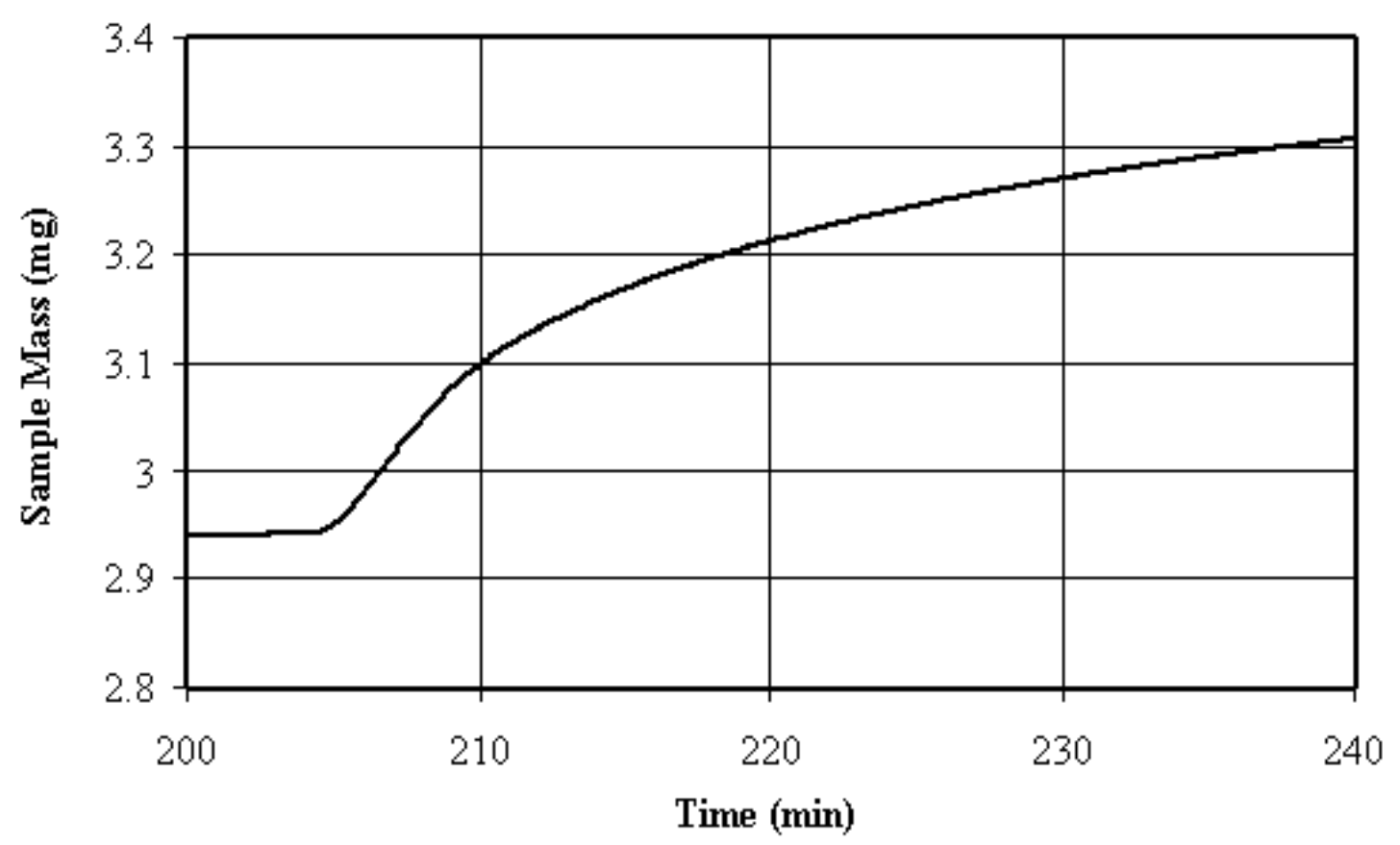

Figure 4.1: Weight Change for Zinc Oxide TGA runs at $60 \%, 1100^{\circ} \mathrm{F}$ and $1 \% \mathrm{H}_{2} \mathrm{~S}$

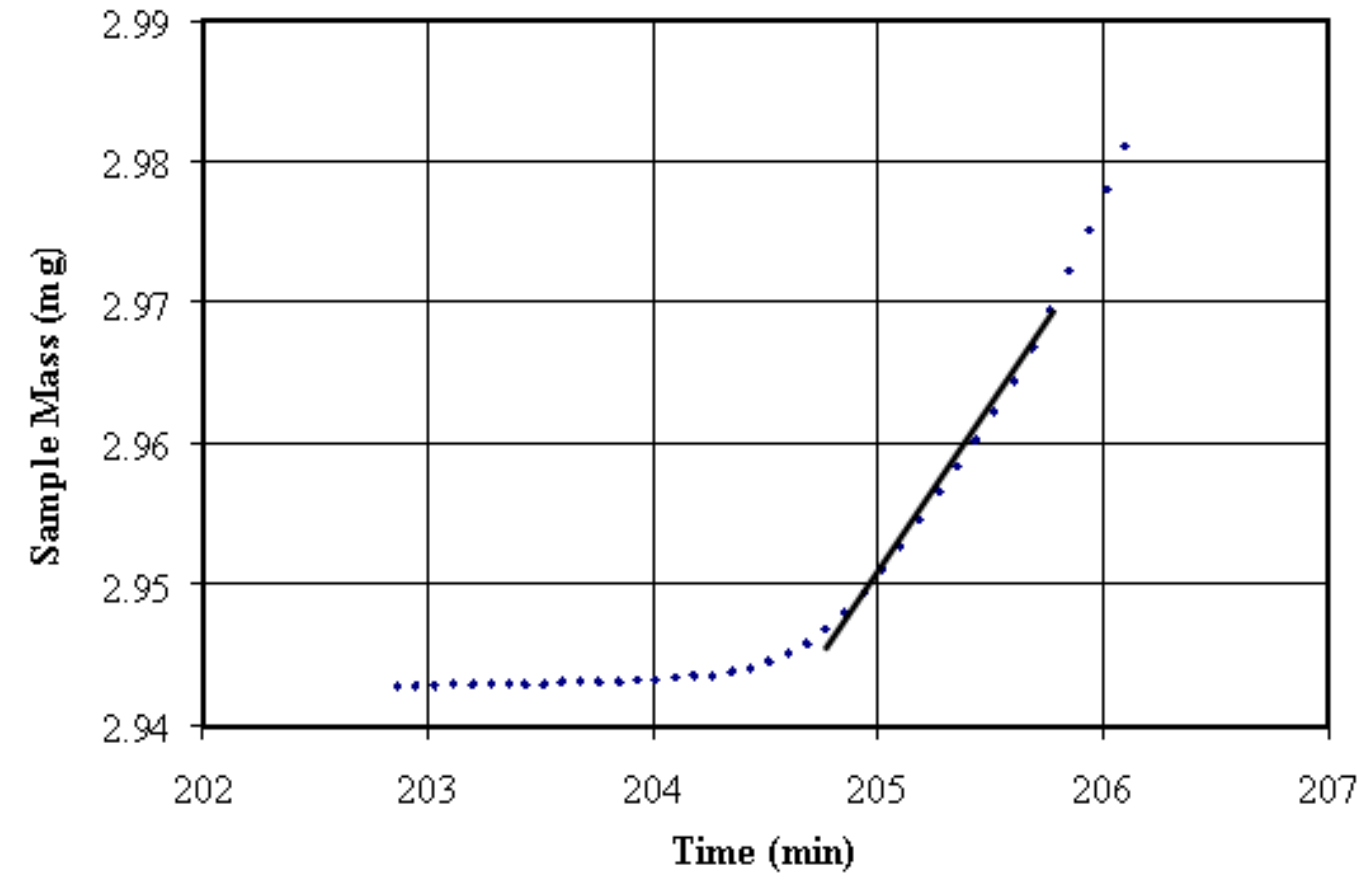

Figure 4.2: Expanded View of Figure 4.1 at the time when $\mathrm{H}_{2} \mathrm{~S}$ is injected into the System 


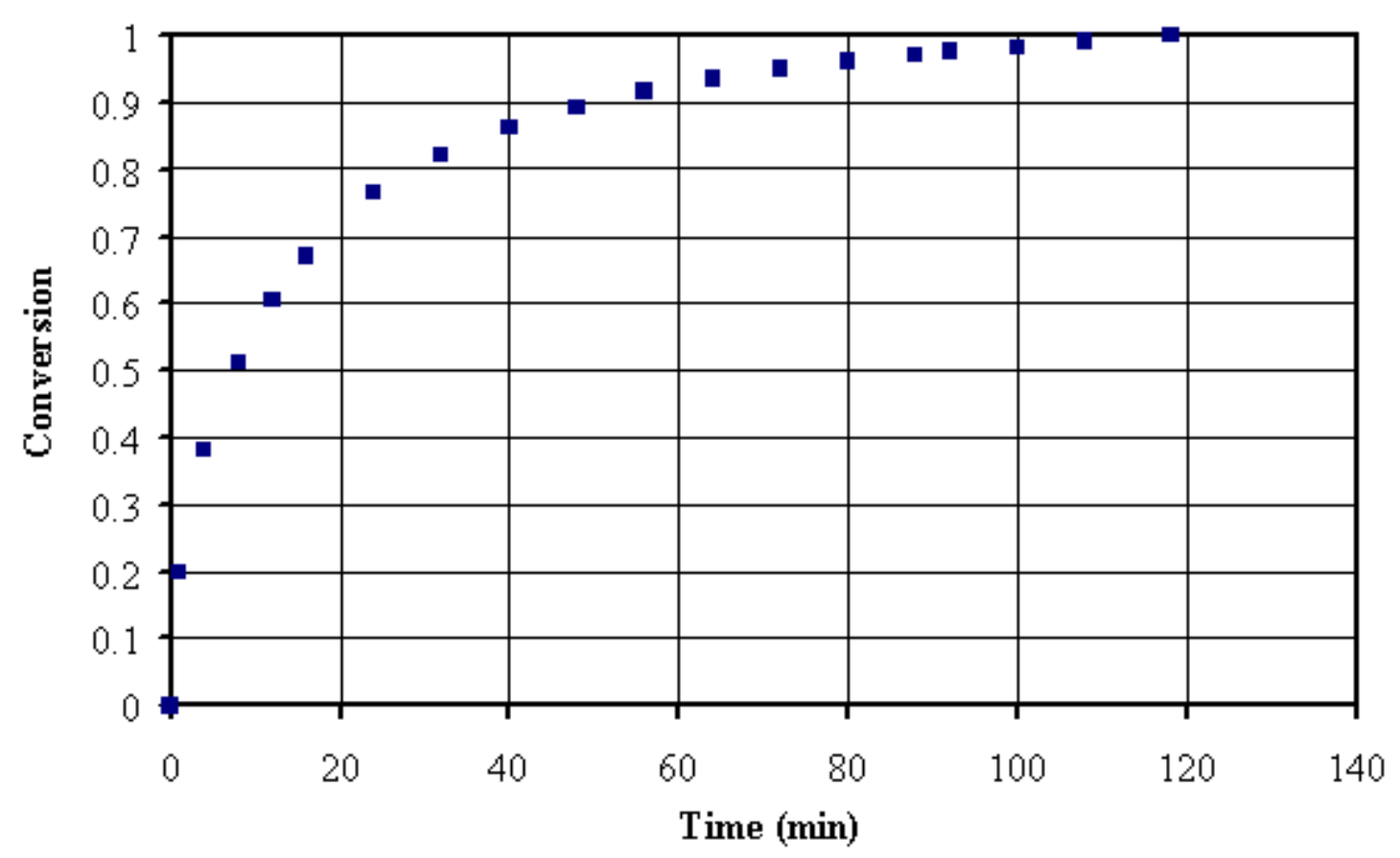

Figure 4.3: Conversion history profile $\left(60 \%, 1100{ }^{\circ} \mathrm{F}, 1 \% \mathrm{H}_{2} \mathrm{~S}\right.$ concentration)

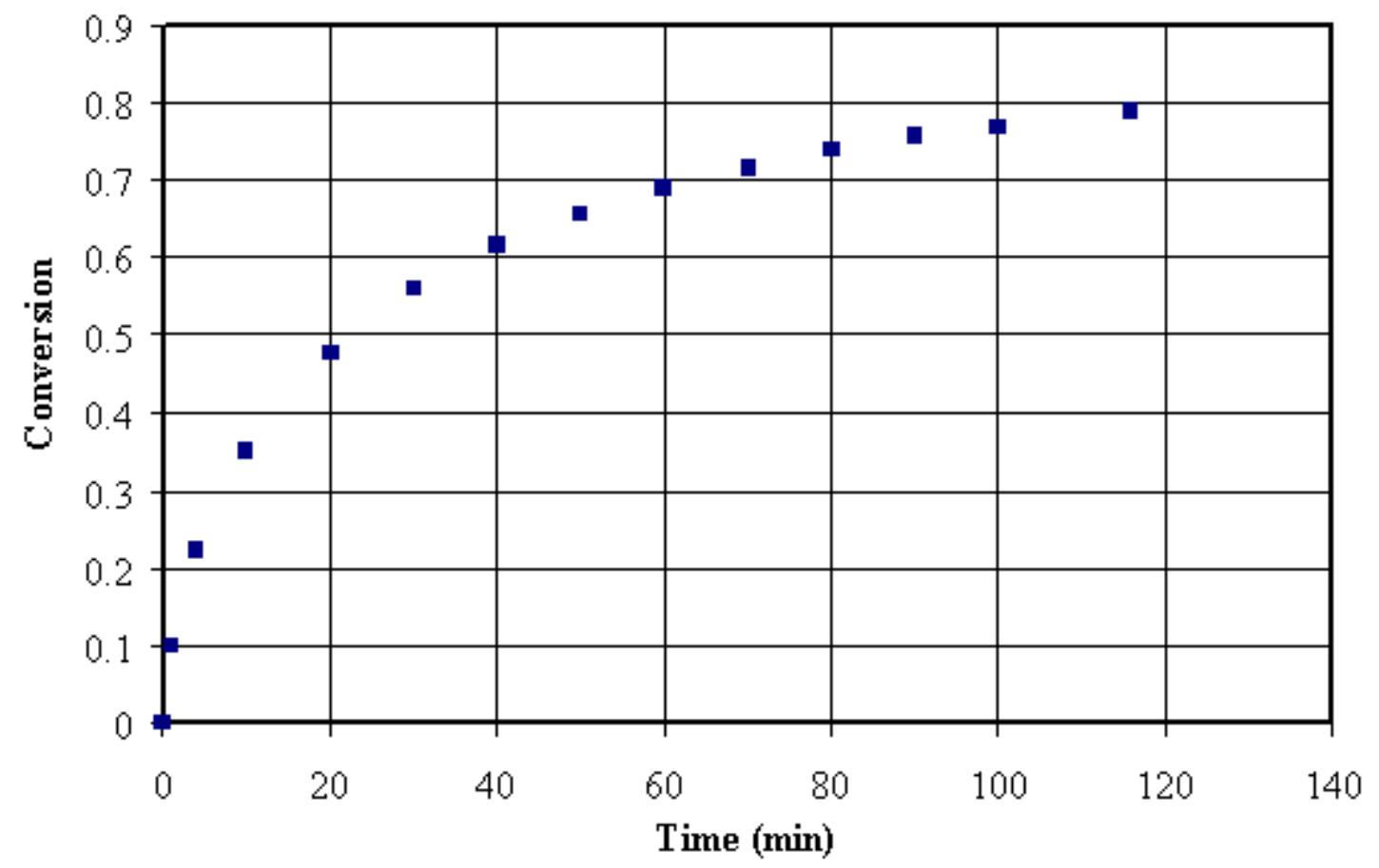

Figure 4.4: Conversion history profile $\left(60 \%, 900{ }^{\circ} \mathrm{F}, 1 \% \mathrm{H}_{2} \mathrm{~S}\right.$ concentration) 


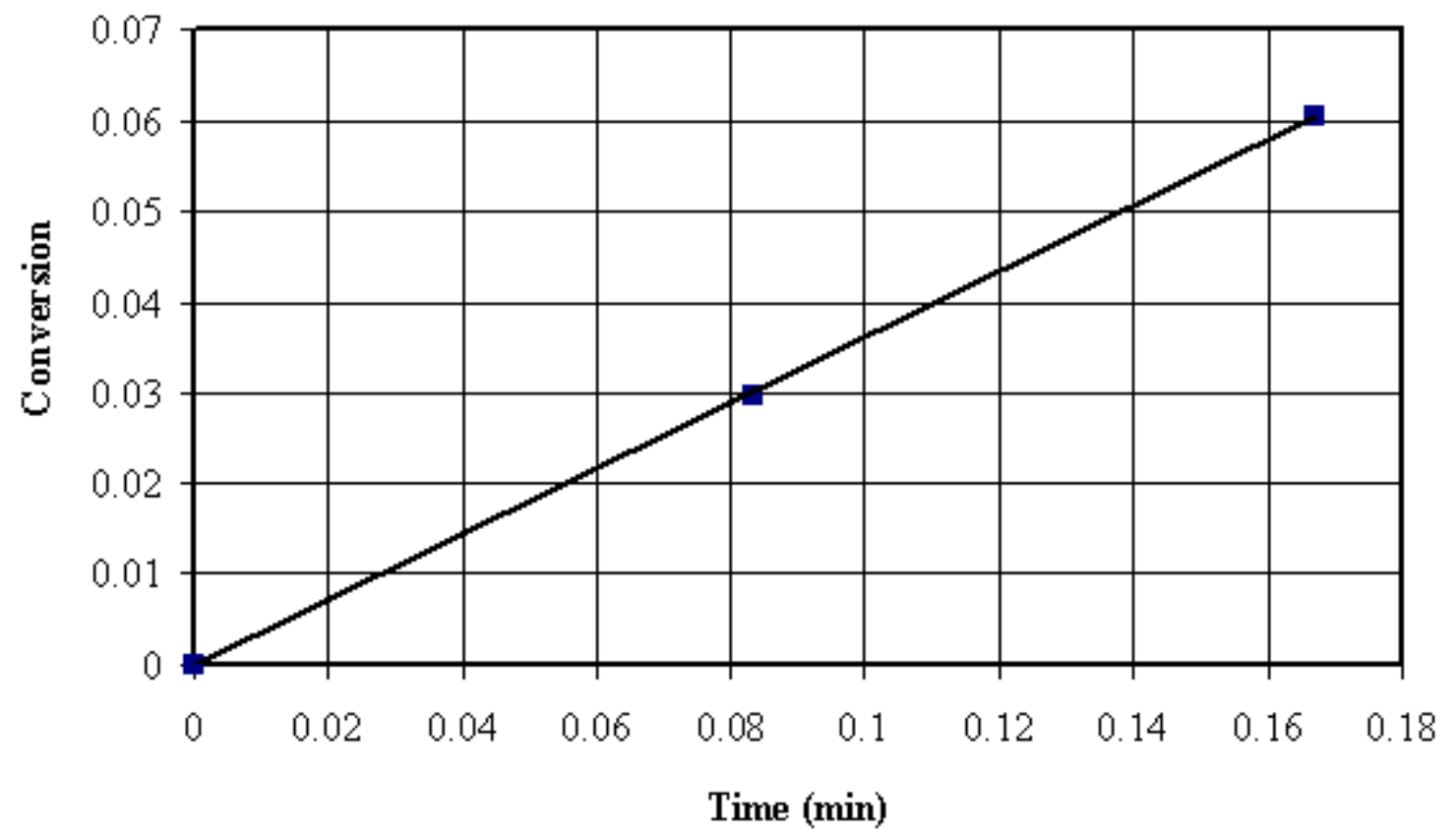

Figure 4.5: Initial conversion history $\left(60 \%, 1100{ }^{\circ} \mathrm{F}, 1 \% \mathrm{H}_{2} \mathrm{~S}\right.$ concentration)

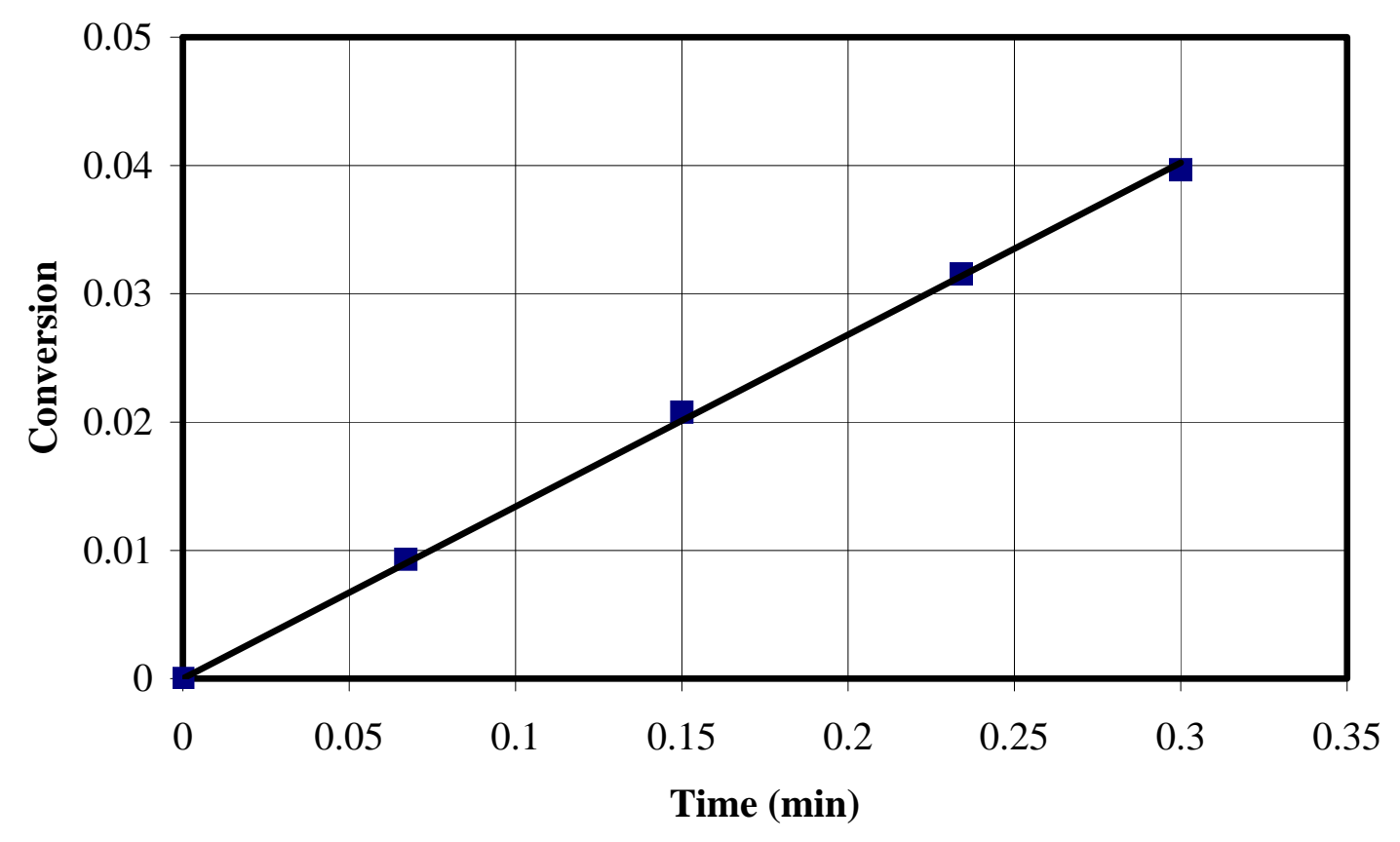

Figure 4.6: Initial Conversion history $\left(60 \%, 900{ }^{\circ} \mathrm{F}, 1 \% \mathrm{H}_{2} \mathrm{~S}\right.$ concentration) 


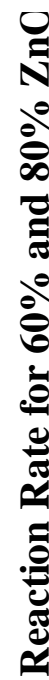

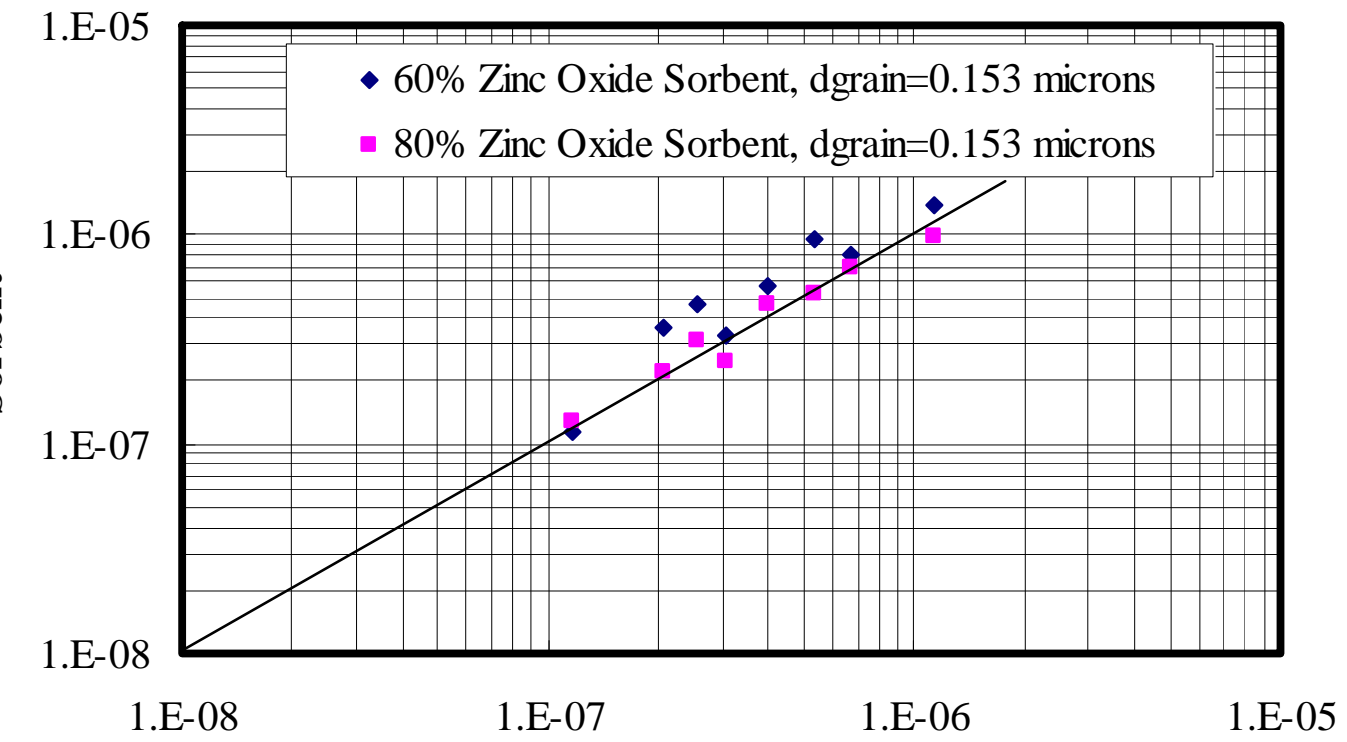

Initial Reaction Rate for $100 \%$ Fresh Sorbent

Figure 4.7: Initial Reaction Rate Comparison for Three Sorbents Used assuming the same grain size

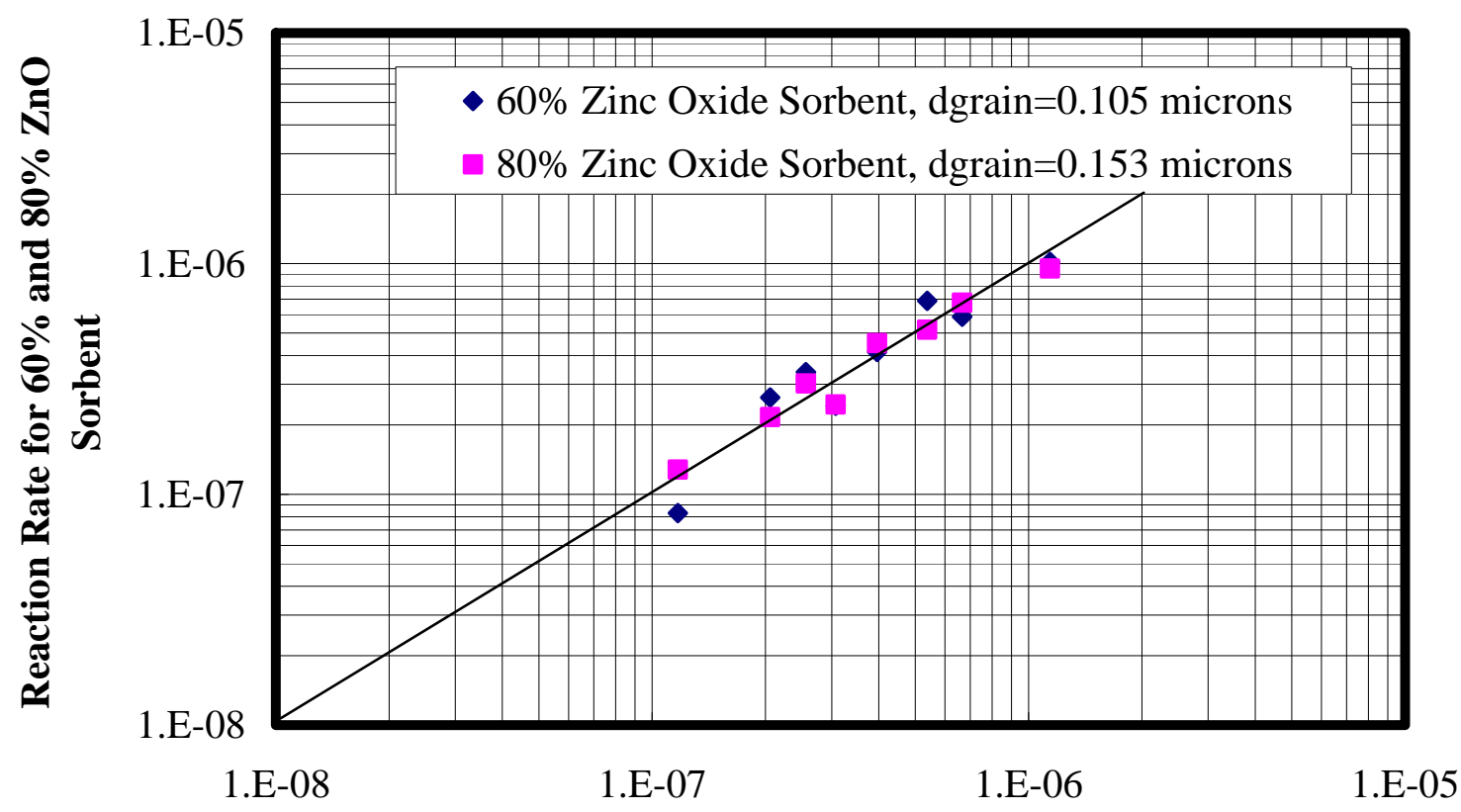

Initial Reaction Rate for $100 \%$ Fresh Sorbent

Figure 4.8: Initial Reaction Rate Comparison for Three Sorbents Used $d_{\text {grain }}=0.105 \mu m$ for $60 \%$ sorbent 


\subsection{Image Analysis}

To obtain grain size distributions, sorbents were analyzed using SEM (Scanning Electron Miscrscope) (SEM model S-4700, Instruments, Hitachi, Ltd.). An image for the $60 \%$ fresh sorbent is given in Figure 4.9.

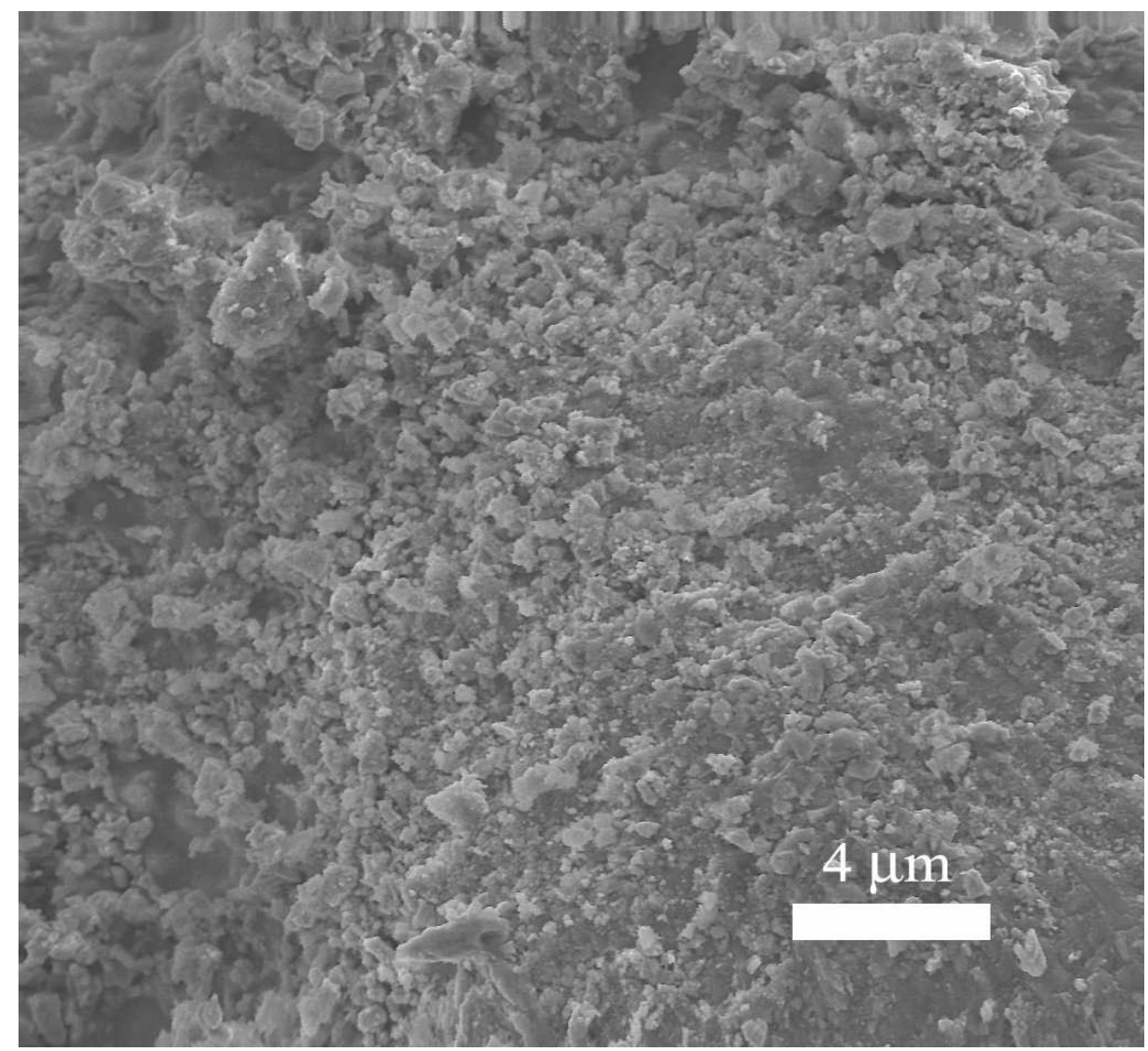

\section{Figure 4.9: SEM image of $60 \%$ fresh sorbent}

We use image analysis software, Visilog ${ }^{\mathrm{TM}}$ (Noesis Vision Inc.), to analyze this image. This software first smoothens the image by softening the edges of objects by filtering high frequencies and noise. It also fills the holes inside the particles, eliminates the small details by smoothing the boundary from the outside and connects close particles. Then it removes isolated points and small particles, shrinks other particles, discards peaks on the boundaries of objects, and disconnects some particles. Finally, it rebuilds the 
image starting from markers and retrieving only the objects containing a marker. After all these steps, we get the result shown in Figure 4.10.

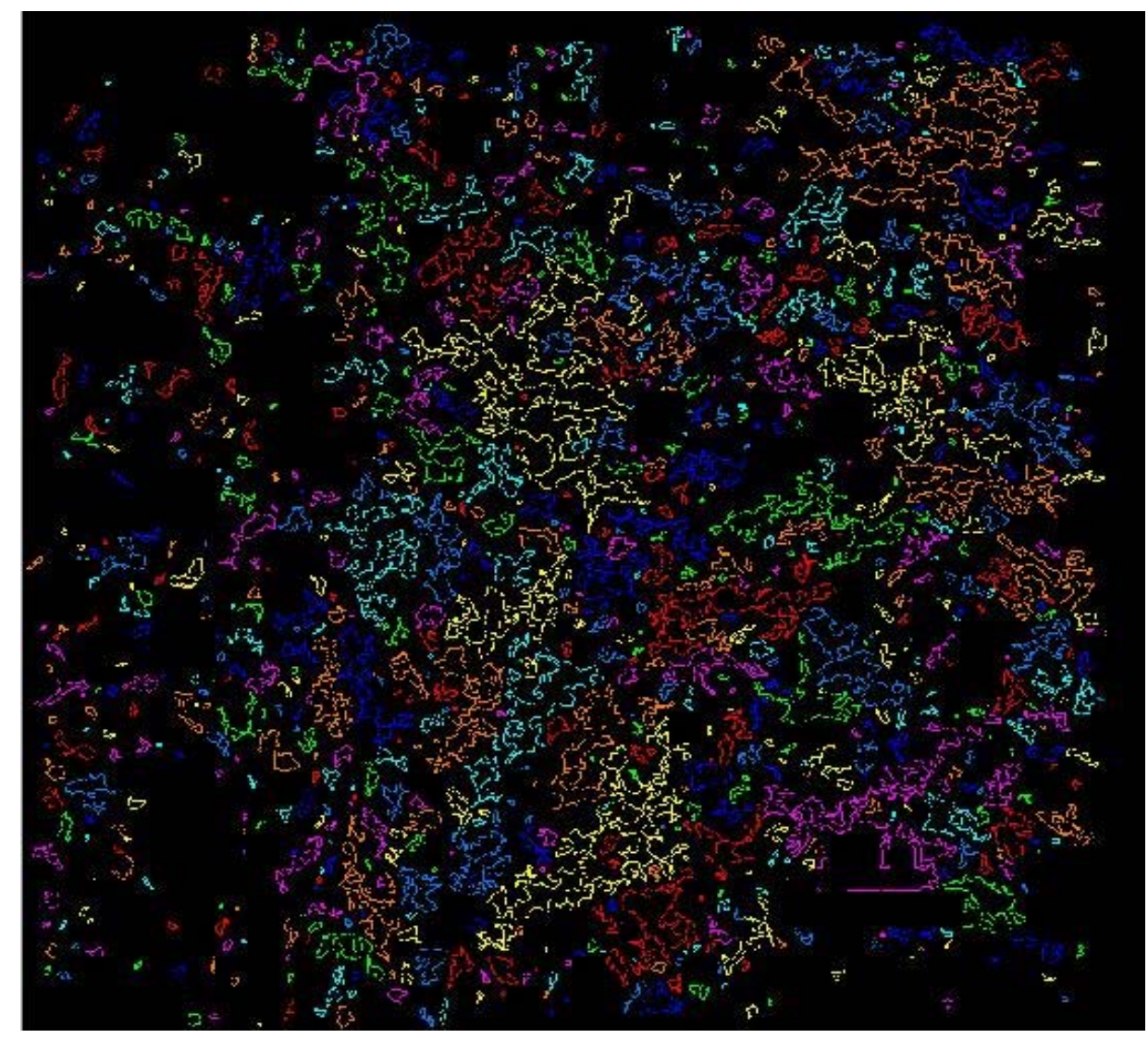

Figure 4.10: Visilog ${ }^{\mathrm{TM}}$ analysis result of SEM image

Also, Visilog ${ }^{\mathrm{TM}}$ will give the number of holes (particles) and the area and perimeter of the holes. From that, we can estimate the size distribution of grains contained in the SEM image. 


\section{RESULTS AND DISCUSSION}

\subsection{Determination of the intrinsic reaction constant}

In order to check the assumption that the gas phase concentration of $\mathrm{H}_{2} \mathrm{~S}$ was constant in the TGA, the following analysis was carried out.

From the intrinsic kinetics of the reaction, at the initial stage of reaction,

$$
-r_{Z n O, o}=\left.\frac{1}{S_{A} M_{Z n O}} \frac{d X}{d t}\right|_{t=0} \quad \mathrm{mmol} / \mathrm{cm}^{2} \cdot \min
$$

Here, $S_{A}$ is the total reaction surface area which is equal to the surface area of the grains. For $1100^{\circ} \mathrm{F}(866 \mathrm{~K}), 1 \% \mathrm{H}_{2} \mathrm{~S}$ concentration and $60 \%$ sorbent case:

Inlet $\mathrm{H}_{2} \mathrm{~S}$ concentration $=\frac{0.01 \times P}{R T}=\frac{0.01 \times 1.013 \times 10^{5}}{8.314 \times 866}=1.406 \times 10^{-4} \quad \mathrm{mmol} / \mathrm{cm}^{3}$

Flow rate of gas $=140 \quad \mathrm{~cm}^{3} / \mathrm{min}$

So, molar flow rate of gas into reactor $=140 \times 1.406 \times 10^{-4}=1.9684 \times 10^{-2} \mathrm{mmol} / \mathrm{min}$

Initial rate of reaction of $\mathrm{ZnO}=\left.\frac{1}{M_{\mathrm{ZnO}}} \frac{d X}{d t}\right|_{t=0}=\frac{1}{0.0814} 0.1633=2.0061 \quad \mathrm{mmol} / \mathrm{g} \cdot \mathrm{min}$

The mass of $\mathrm{ZnO}=0.6 \times 2.5713=1.5428 \mathrm{mg}=1.5428 \times 10^{-3} \quad \mathrm{~g}$

And, $\mathrm{H}_{2} \mathrm{~S}$ reacted in reactor $=2.0061 \times 1.5428 \times 10^{-3}=3.0950 \times 10^{-3} \quad \mathrm{mmol} / \mathrm{min}$

Thus, $\mathrm{H}_{2} \mathrm{~S}$ conversion in gas steam $=\frac{3.0950 \times 10^{-3}}{1.9684 \times 10^{-2}}=16 \%$

Because $16 \%$ conversion is the maximum $\mathrm{H}_{2} \mathrm{~S}$ conversion (initial reaction) for the whole reaction process, the assumption of constant $C_{\mathrm{H}_{2} \mathrm{~S}}$ is reasonable.

From experimental data, we can find the initial reaction rate $-r_{Z n O, o}$ and $C_{\mathrm{H}_{2} \mathrm{~S}}$ for different operating conditions and combining Equations (5) and (37), to give: 


$$
\ln \left(\frac{-r_{\mathrm{ZnO}, \mathrm{o}}}{C_{\mathrm{H}_{2} \mathrm{~S}}}\right)=\ln A-\frac{E}{R}\left(\frac{1}{T}\right)
$$

The best fit between Equation (38) and the experimental data is found using the following rate constants and the result is shown in Figure 5.1.

$$
\begin{aligned}
& k_{1100}=0.214 \quad \mathrm{~cm} / \mathrm{min} \\
& k_{900}=0.112 \quad \mathrm{~cm} / \mathrm{min}
\end{aligned}
$$

From this data, we estimate the activation energy of the reaction $E$ as $7.5 \mathrm{kcal} / \mathrm{mol}$, which is consistent with the work of Westmoreland [5].

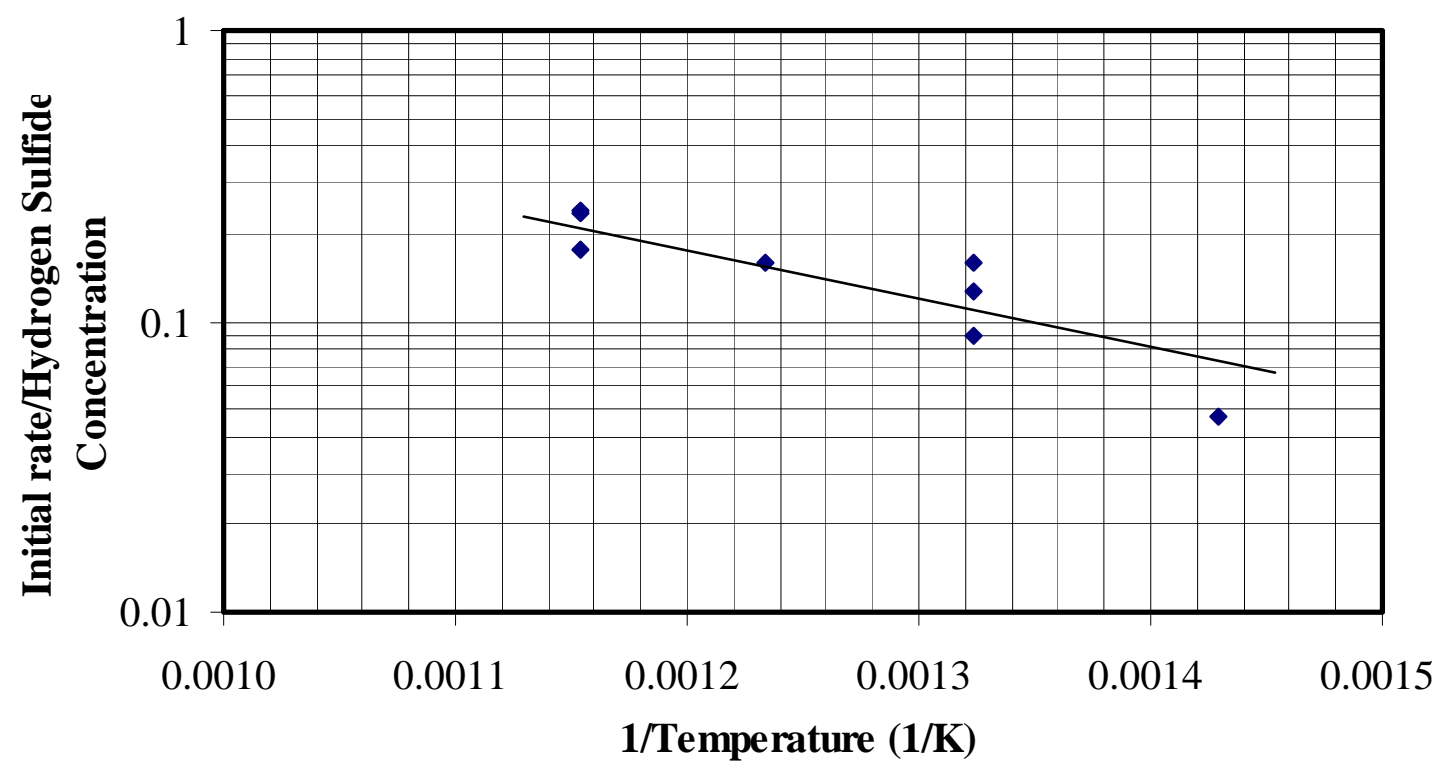

Figure 5.1: Comparison of Model Predictions with Experimental Data

\subsection{Predicting conversion using SCM model}

\subsubsection{Constant Pellet Size}

For the SCM model, we can rewrite Equation (13): 


$$
t=\frac{\rho_{B} R}{3 k_{g} C_{A b} M_{B}} X+\frac{\rho_{B} R^{2}}{6 D_{e} C_{A b} M_{B}}\left[1-3(1-X)^{2 / 3}+2(1-X)\right]+\frac{\rho_{B} R}{k C_{A b} M_{B}}\left[1-(1-X)^{1 / 3}\right]
$$

Here, $k_{g}$ is the external mass transfer coefficient and $D_{e}$ is the effective diffusivity in the product layer.

We can approximate the external mass transfer coefficient using the following expressing due to Froessling [21]:

$$
S h=\frac{d_{p} k_{g}}{D_{H_{2} S-G a s}}=2.0+0.6 \operatorname{Re}^{1 / 2} S c^{1 / 3}
$$

For small particles, we may consider that

$$
S h=\frac{d_{p} k_{g}}{D_{H_{2} S-G a s}}=2.0
$$

The diffusion coefficient for $\mathrm{H}_{2} \mathrm{~S}$ in the gas was estimated using the following equation due to Hirschfelder et al. [22]:

$$
D_{A-B}=0.001858 \frac{T^{3 / 2}\left(\frac{1}{M_{A}}+\frac{1}{M_{B}}\right)^{1 / 2}}{P \sigma_{A B}^{2} \Omega_{D}} \quad \mathrm{~cm}^{2} / \mathrm{s}
$$

Here for our system $\left(1100^{\circ} \mathrm{F}\right.$ case):

$$
\begin{aligned}
& T=1100^{\circ} \mathrm{F}=866 \mathrm{~K} \\
& P=1.013 \times 10^{5} \mathrm{~Pa} \\
& M_{A}=28 \\
& M_{B}=34
\end{aligned}
$$




$$
\begin{aligned}
& \sigma_{A B}^{2}=\left(\frac{\left.(3.623+3.798)^{2}\right)}{2}\right)^{2}=13.77 \AA \\
& \frac{\varepsilon_{A B}}{k T}=\frac{[(301.1)(71.4)]^{1 / 2}}{866}=0.169 \Rightarrow \frac{k T}{\varepsilon_{A B}}=5.9 \\
& \Omega_{D}=f\left(\frac{k T}{\varepsilon_{A B}}\right)=0.82
\end{aligned}
$$

So, $D_{H_{2} S-G a s}=0.273 \mathrm{~cm}^{2} / \mathrm{s}$

Then, $k_{g}=2 \frac{D_{H_{2} s-G a s}}{d_{p}}=2 \frac{0.273 \times 10^{-4}}{75 \times 10^{-6}}=0.728 \mathrm{~m} / \mathrm{s} \quad>\quad k_{1100}=0.356 \times 10^{-6} \mathrm{~m} / \mathrm{s}$

By comparing the relative magnitudes of $k_{g}$ and $k$, we concluded that the external mass transfer is negligible, so Equation (13) is reduced to:

$$
t=\frac{\rho_{Z n O} R^{2}}{6 D_{e} C_{A b} M_{Z n O}}\left[1-3(1-X)^{2 / 3}+2(1-X)\right]+\frac{\rho_{Z n O} R}{k C_{A b} M_{Z n O}}\left[1-(1-X)^{1 / 3}\right]
$$

or

$$
\begin{gathered}
t=\tau_{\text {ash }}\left[1-3(1-X)^{2 / 3}+2(1-X)\right]+\tau_{\text {reac }}\left[1-(1-X)^{1 / 3}\right] \\
\tau_{\text {ash }}=\frac{\rho_{B} R^{2}}{6 D_{e} C_{A b} M_{B}} \quad \tau_{\text {reac }}=\frac{\rho_{B} R}{k C_{A b} M_{B}}
\end{gathered}
$$

For the run at $593^{\circ} \mathrm{C}\left(1100^{\circ} \mathrm{F}\right)$ and $1 \% \mathrm{H}_{2} \mathrm{~S}$ concentration, we know:

The time for complete reaction is: $\mathrm{t}=118.085 \quad \min$

$$
\begin{array}{llll}
k_{1100}=0.214 & \mathrm{~cm} / \mathrm{min} & C_{A b}=1.406 \times 10^{-4} & \mathrm{mmol} / \mathrm{cm}^{3} \\
M_{B}=0.0814 & \mathrm{~g} / \mathrm{mmol} & \rho_{B}=5.6 & \mathrm{~g} / \mathrm{cm}^{3}
\end{array}
$$


$R=\frac{d_{g}}{2}=7.642 \times 10^{-6} \quad \mathrm{~cm}$

So, $\tau_{\text {reac }}=\frac{\rho_{B} R}{k C_{A b} M_{B}}=\frac{5.6 \cdot 7.642 \times 10^{-6}}{0.214 \cdot 1.406^{-4} \cdot 0.0814}=17.505 \quad$ min

Then, at $X=1: t=\tau_{\text {ash }}+\tau_{\text {reac }} \Rightarrow \tau_{\text {ash }}=t-\tau_{\text {reac }}=100.58 \quad$ min

Thus, $D_{e}=\frac{\rho_{B} R}{6 \tau_{a s h} C_{A b} M_{B}}=4.734 \times 10^{-8} \quad \quad \mathrm{~cm}^{2} / \mathrm{min}$

After we estimate $\tau_{\text {ash }}$ and $\tau_{\text {reac }}$, we can use Equation (42) to plot the conversion-time profile and compare it with the experimental data. This procedure was adopted for all experiments. A typical result is shown in Figure 5.2.

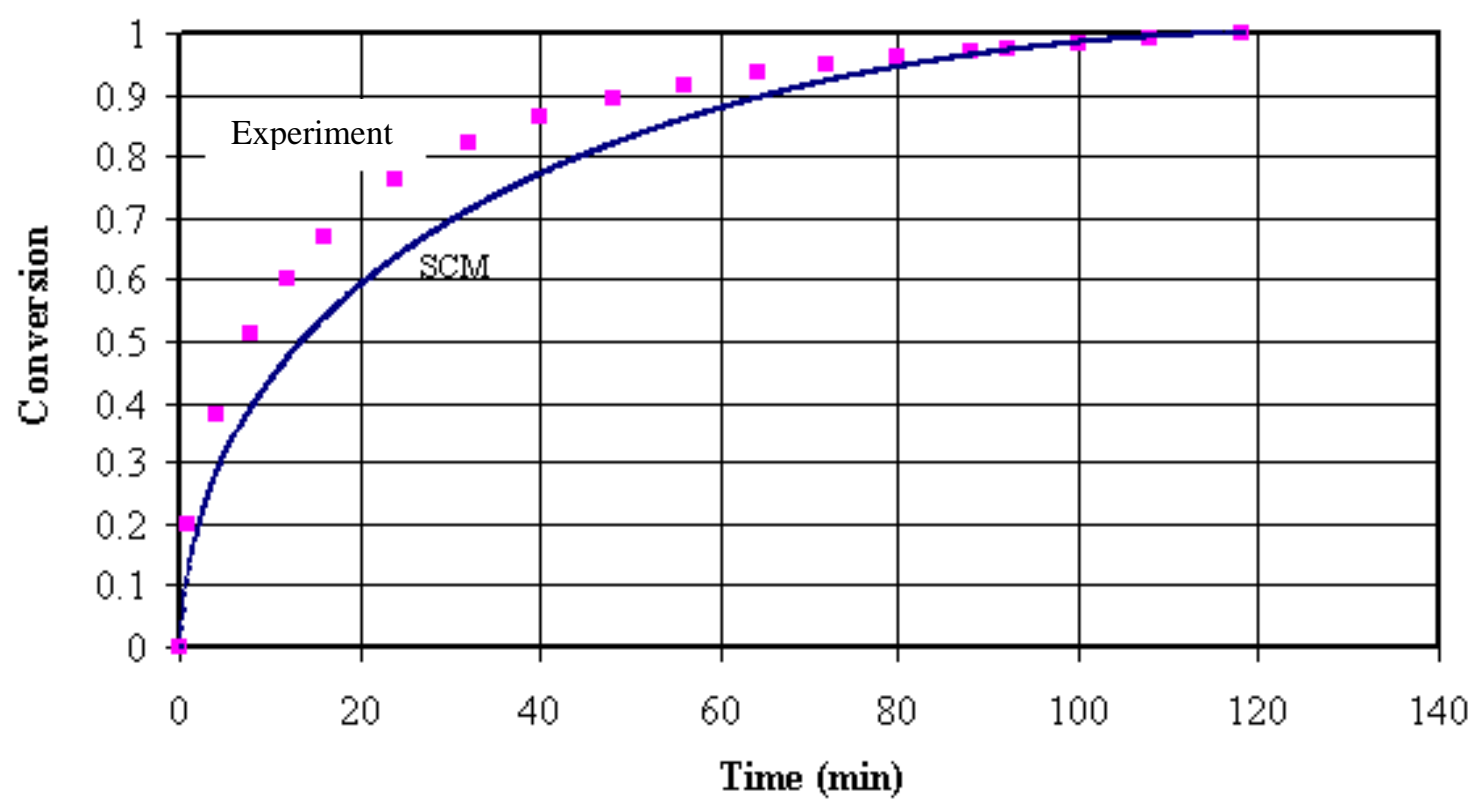

Figure 5.2: Experimental results compared with prediction of SCM model $(60 \%$, $593^{\circ} \mathrm{C}, 1 \% \mathrm{H}_{2} \mathrm{~S}$ concentration and $d_{g}=1.53 \times 10^{-5} \mathrm{~cm}$ )

From the Figure 5.2, we see that the SCM model systematically under-predicts the experimental data. The only fitting parameter, $D_{e}$, for this model is also fixed from the 
total reaction time. The result in Figure 14 shows a systematic underprediction by the basic SCM.

\subsubsection{Changing Pellet Size due to Volume Change}

Recalling Equation (25),

$$
-r_{A}=4 \pi R_{o}^{2}\left[\frac{1}{\left[Z_{v}+\left(1-Z_{v}\right)(1-X)\right]^{2 / 3} k_{g}}+\frac{R_{o}\left[\left[Z_{v}+\left(1-Z_{v}\right)(1-X)\right]^{1 / 3}-(1-X)^{1 / 3}\right]}{\left[Z_{v}+\left(1-Z_{v}\right)(1-X)\right]^{1 / 3}(1-X)^{1 / 3} D_{e}}+\frac{1}{(1-X)^{2 / 3} k}\right]^{-1} \cdot C_{A b}
$$

Since the external mass transfer is negligible, the equation above becomes:

$$
-r_{A}=4 \pi R_{o}^{2}\left[\frac{R_{o}\left[\left[Z_{v}+\left(1-Z_{v}\right)(1-X)\right]^{1 / 3}-(1-X)^{1 / 3}\right]}{\left[Z_{v}+\left(1-Z_{v}\right)(1-X)\right]^{1 / 3}(1-X)^{1 / 3} D_{e}}+\frac{1}{(1-X)^{2 / 3} k}\right]^{-1} \cdot C_{A b}
$$

Also, $-r_{Z n O}=\frac{1}{S_{A} M_{Z n O}} \frac{d X}{d t}$

Combining Equation (43) and Equation (44) and integrating, we get:

$$
t=\frac{\rho_{Z n O} R_{o}{ }^{2}}{2 D_{e} C_{A b} M_{Z n O}}\left[\frac{Z_{v}-\left[Z_{v}+\left(1-Z_{v}\right)(1-X)\right]^{2 / 3}}{Z_{v}-1}-(1-X)^{2 / 3}\right]+\frac{\rho_{Z n O} R_{o}}{k C_{A b} M_{Z n O}}\left[1-(1-X)^{1 / 3}\right]
$$

Using Equation (45), we can predict the conversion-time profile and compare it with the experimental results. Figure 5.3 shows this comparision using a value of $Z_{v}=1.64$, that is consistent with the $\mathrm{ZnO}-\mathrm{ZnS}$ system.

The results in Figure 5.3 show that the modified SCM gives worse predictions than the basic SCM model. By introducing $Z_{v}$ into the models, we increase the ash layer diffusion path, so this result is not surprising. 


\subsection{Predicting conversion using Basic GPM model}

Compared with SCM, which has three reaction resistances: external mass transfer, ash layer diffusion and surface chemical reaction, the basic GPM has one more resistance:

intra-pellet diffusion. If the intra-pellet diffusion is significant, the conversion profiles should be dependent on the pellet size.

The conversion-time data for different pellet sizes is shown in Figure 5.4. From the plot, it is clear that conversion vs. time data are almost identical for different pellet sizes. So for this project, the intra-pellet diffusion is assumed to be negligible. Thus, for the current case, the basic GPM is the same as the SCM except that the charactertic length is the grain diameter.

If we use the effective average grain dimension (for $60 \%$ sorbent), that is:

$$
\bar{d}_{g}=1.05 \times 10^{-5} \quad \mathrm{~cm}
$$

From Equation (42),

$$
t=\frac{\rho_{\mathrm{ZnO}} R^{2}}{6 D_{e} C_{A b} M_{\mathrm{ZnO}}}\left[1-3(1-X)^{2 / 3}+2(1-X)\right]+\frac{\rho_{\mathrm{ZnO}} R}{k C_{A b} M_{Z n O}}\left[1-(1-X)^{1 / 3}\right]
$$

The reaction history profile was obtained using Equation (42) and described previously in the SCM. This profile is shown in Figure 5.5.

If we consider an expanding grain using Equation (45), the results are shown in Figure5.6.

$$
t=\frac{\rho_{\mathrm{ZnO}} R_{o}{ }^{2}}{2 D_{e} C_{A b} M_{Z n O}}\left[\frac{Z_{v}-\left[Z_{v}+\left(1-Z_{v}\right)(1-X)\right]^{2 / 3}}{Z_{v}-1}-(1-X)^{2 / 3}\right]+\frac{\rho_{Z n O} R_{o}}{k C_{A b} M_{Z n O}}\left[1-(1-X)^{1 / 3}\right]
$$

From Figures 5.2, 5.3, 5.5 and 5.6, we conclude that neither the basic SCM nor the basic GPM can predict the experimental data very well. The reason is, as mentioned before,that 
the only fitting parameter, $D_{e}$, is also fixed by matching the total reaction time for complete conversion. Therefore, the basic grain GPM and SCM models systematically underpredict the conversion-time data.

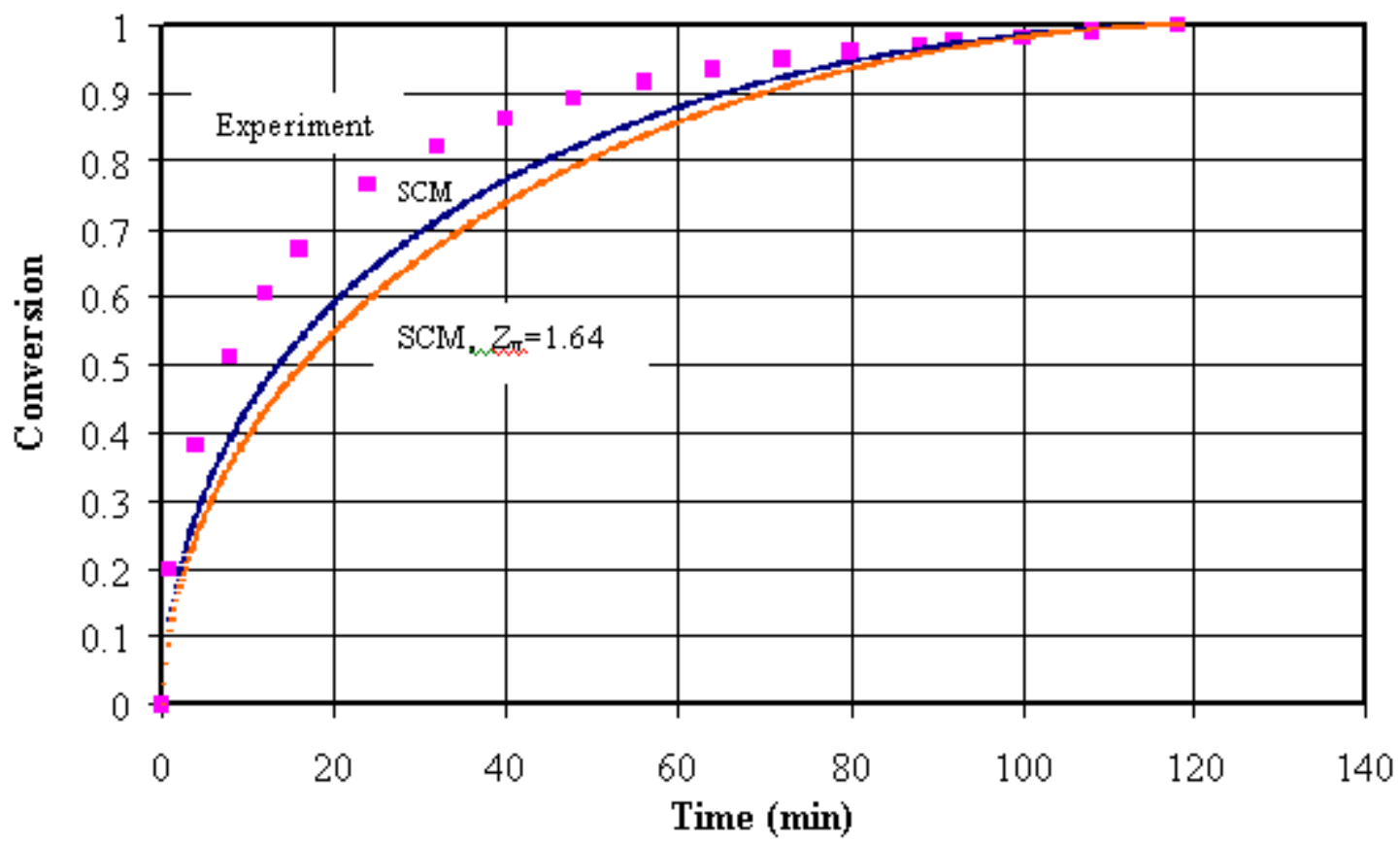

Figure 5.3:Experimental results compared with predictions of SCM with and without $Z_{v}\left(60 \%, 593{ }^{\circ} \mathrm{C}, 1 \% \mathrm{H}_{2} \mathrm{~S}\right.$ concentration and $\left.d_{g}=1.53 \times 10^{-5} \mathrm{~cm}\right)$ 


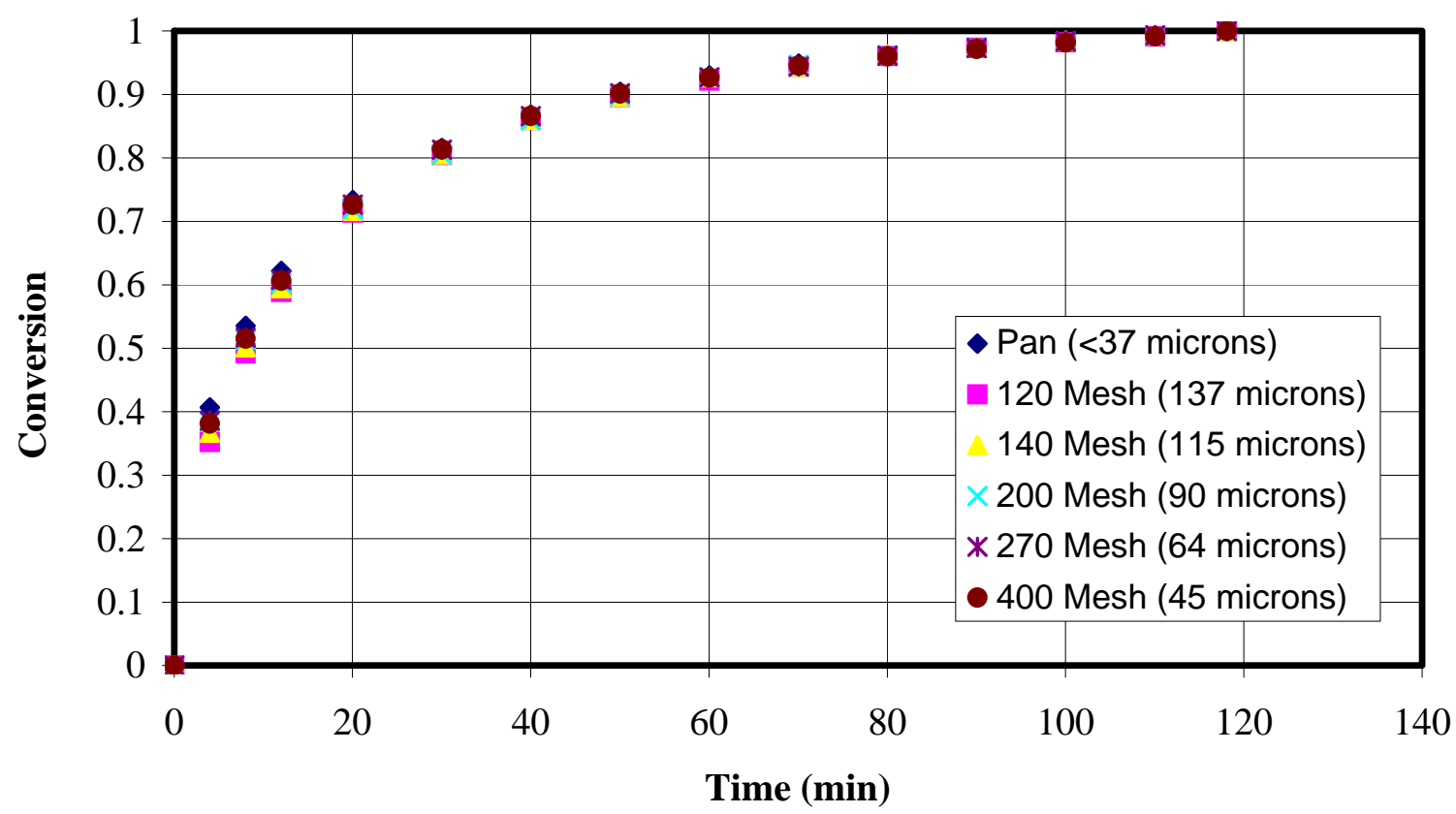

Figure 5.4: Conversion profile for different pellet sizes for $60 \%$ sorbent, $593{ }^{\circ} \mathrm{C}, 1 \%$ $\mathrm{H}_{2} \mathrm{~S}$ concentration

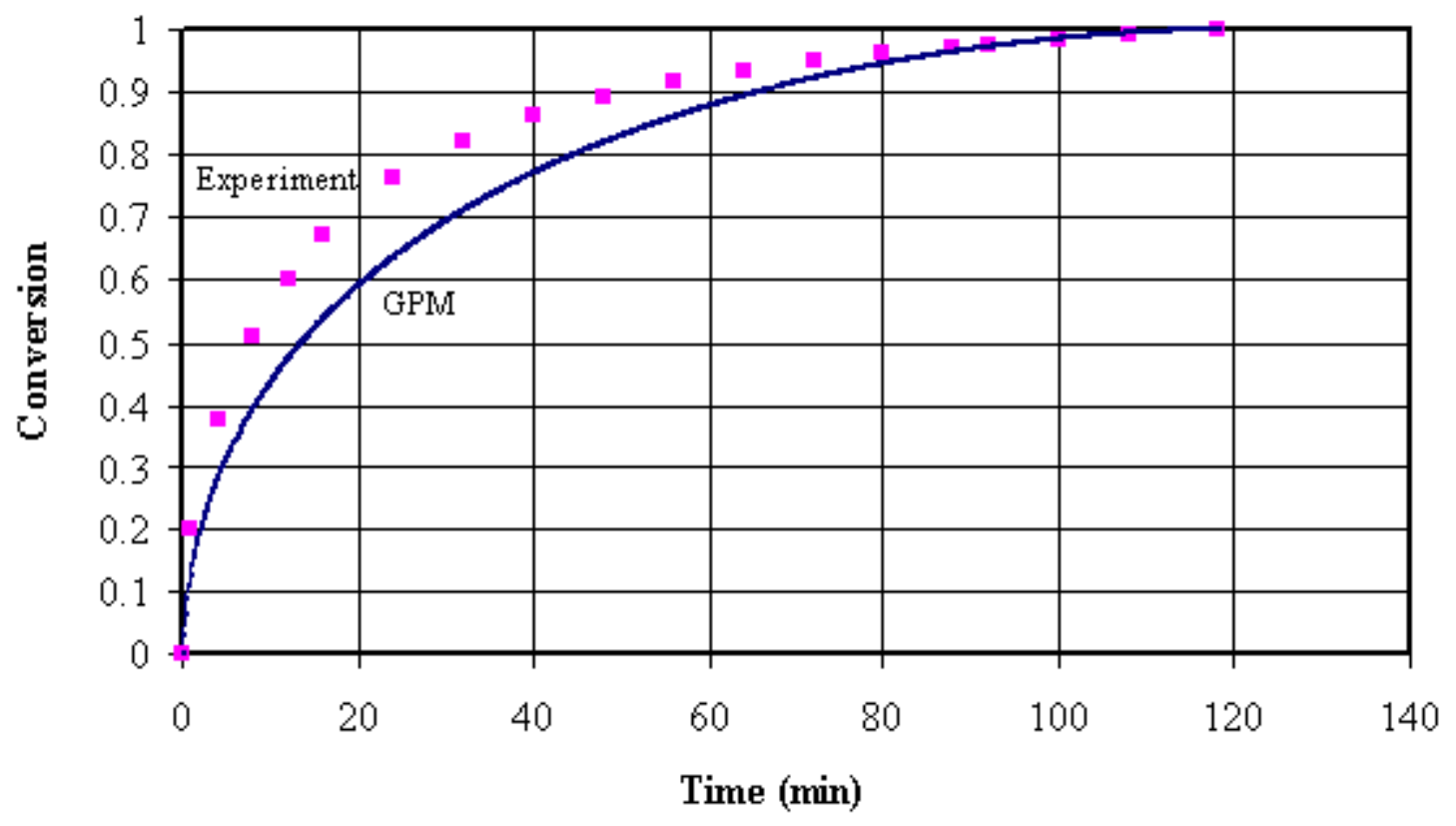

Figure 5.5: Basic GPM compared with experimental data $\left(60 \%, 593{ }^{\circ} \mathrm{C}, 1 \% \mathrm{H}_{2} \mathrm{~S}\right.$ concentration and $d_{g}=1.05 \times 10^{-5} \mathrm{~cm}$ ) 


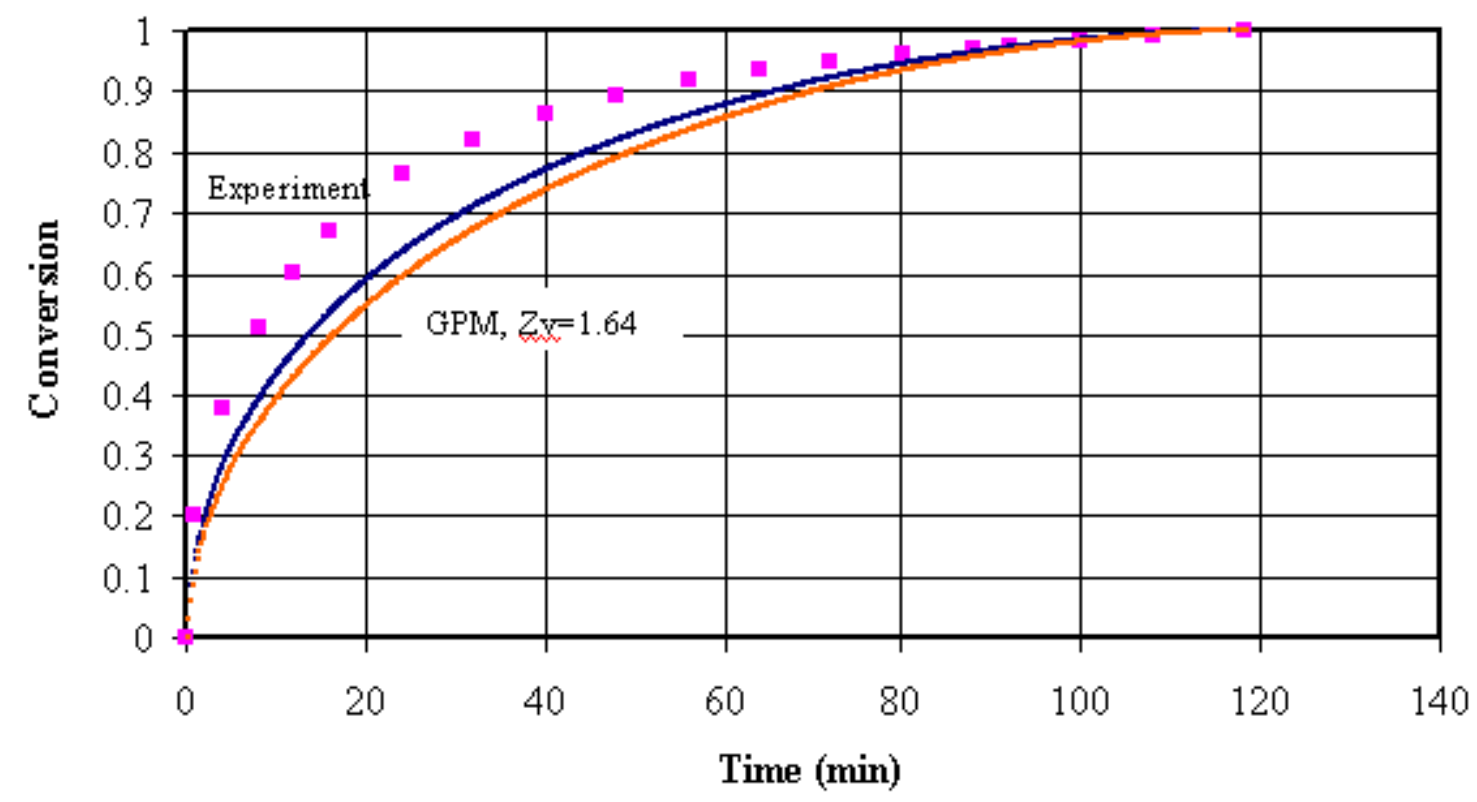

Figure 5.6: Experimental results compared with basic GPM with and without $Z_{v}$ $\left(60 \%, 593{ }^{\circ} \mathrm{C}, 1 \% \mathrm{H}_{2} \mathrm{~S}\right.$ concentration and $\left.d_{g}=1.05 \times 10^{-5} \mathrm{~cm}\right)$

\subsection{GPM Model using a Grain Size Distribution}

\subsubsection{GPM Model using a Bimodal Grain Size}

From the previous section, we know that the basic GPM always underpredicts the experimental data. So, we assume the $\mathrm{ZnO}$ grains are composed of two sizes: $d_{g 1}, d_{g 2}$ where $d_{g 1}<d_{g 2}$. Since the small grains react faster than the large grains, by combing two grains' reaction histories, we can get a close match with experimental data.

From Equation (36), we know the total reaction surface area for $60 \mathrm{wt} \%$ sorbent is:

$$
S_{A}=6.09 \times 10^{4} \quad \mathrm{~cm}^{2} / \mathrm{g}
$$

And the grain has a density of:

$$
\rho_{\text {ZnO }}=5.6 \quad \mathrm{~g} / \mathrm{cm}^{3}
$$


Let $x_{1}$ and $x_{2}$ be the mass fraction of grains $d_{g 1}$ and $d_{g 2}$ respectively, $N_{g 1}$ and $N_{g 2}$ are the unit number of grains for $d_{g 1}$ and $d_{g 2}$ respectively.

So the volume and surface area of total $\mathrm{ZnO}$ grains is:

$$
\begin{array}{ll}
V=\frac{1}{\rho}=x_{1} \cdot N_{g 1} \cdot \frac{\pi}{6} d_{g 1}^{3}+x_{2} \cdot N_{g 2} \cdot \frac{\pi}{6} d_{g 2}^{3} & \mathrm{~cm}^{3} / \mathrm{g} \\
S_{A}=x_{1} \cdot N_{g 1} \cdot \pi d_{g 1}^{2}+x_{2} \cdot N_{g 2} \cdot \pi d_{g 2}^{2} & \mathrm{~cm}^{2} / \mathrm{g}
\end{array}
$$

Also, we know that:

$$
\begin{aligned}
& N_{g 1}=\frac{1}{\rho \frac{\pi}{6} d_{g 1}^{3}} \\
& N_{g 2}=\frac{1}{\rho \frac{\pi}{6} d_{g 2}^{3}}
\end{aligned}
$$

By choosing different values of $x_{1}, d_{g 1}$ and combining equations (46), (47), (48) and (49), we can get the corresponding $x_{2}$ and $d_{g 2}$.

From Equation (42):

$$
\begin{gathered}
t=\tau_{\text {ash }}\left[1-3(1-X)^{2 / 3}+2(1-X)\right]+\tau_{\text {reac }}\left[1-(1-X)^{1 / 3}\right] \\
\tau_{\text {ash }}=\frac{\rho_{\mathrm{ZnO}} R^{2}}{6 D_{e} C_{A b} M_{\mathrm{ZnO}}} \quad \tau_{\text {reac }}=\frac{\rho_{\mathrm{ZnO}} R}{k C_{A b} M_{\mathrm{ZnO}}}
\end{gathered}
$$

By definition of $\tau_{\text {ash }}$ and $\tau_{\text {reac }}$ we know:

$$
\tau_{a s h 1}=\tau_{a s h 2}\left(\frac{d_{g 1}}{d_{g 2}}\right)^{2} \quad \tau_{\text {reac1 }}=\tau_{\text {reac } 2}\left(\frac{d_{g 1}}{d_{g 2}}\right)
$$

Since $d_{g 1}<d_{g 2}, \tau_{\text {ash } 2}+\tau_{\text {reac } 2}=$ Reaction time for complete conversion 
From Equation (26), for the case of a distribution of two grain sizes:

$$
X=1-x_{1}\left(1-X_{1}\right)-x_{2}\left(1-X_{2}\right)
$$

Thus from the $X$ vs. $t$ plot for each grain and by using Equation (26), we can obtain the total reaction history profile. The result for $593^{\circ} \mathrm{C}\left(1100^{\circ} \mathrm{F}\right)$ is given in Figure 5.7.

If we consider the change of grain structure, using Equation (45), we get

$$
t=\frac{\rho_{\mathrm{ZnO}} R_{o}{ }^{2}}{2 D_{e} C_{A b} M_{\mathrm{ZnO}}}\left[\frac{Z_{v}-\left[Z_{v}+\left(1-Z_{v}\right)(1-X)\right]^{2 / 3}}{Z_{v}-1}-(1-X)^{2 / 3}\right]+\frac{\rho_{\mathrm{ZnO}} R_{o}}{k C_{A b} M_{\mathrm{ZnO}}}\left[1-(1-X)^{1 / 3}\right]
$$

The result for $593^{\circ} \mathrm{C}$ is given in Figure 5.8. For $482^{\circ} \mathrm{C}\left(900^{\circ} \mathrm{F}\right)$ case, the result is shown in Figure 5.9.

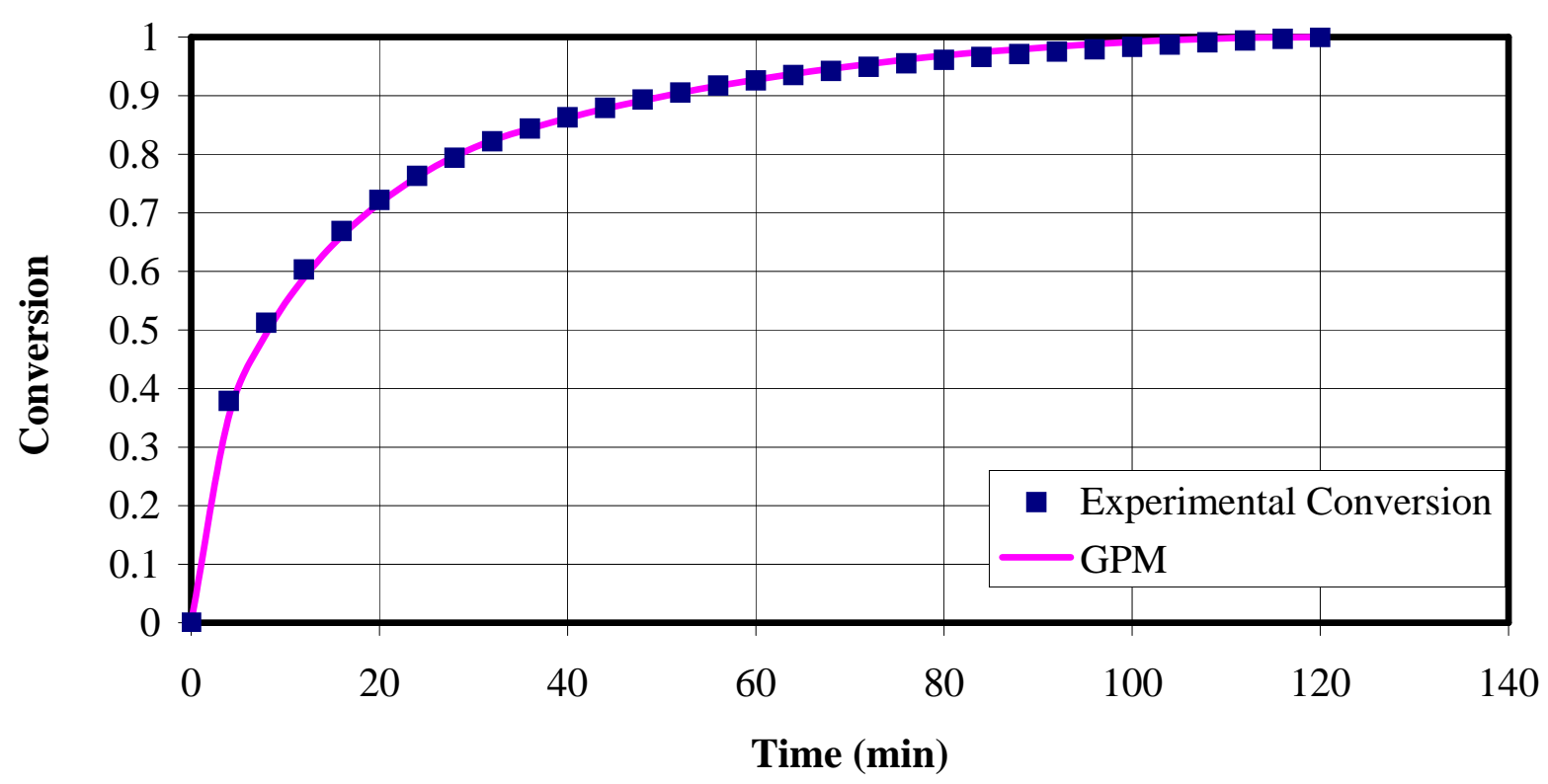

Figure 5.7: Experimental results compared with GPM $\left(x_{1}=0.4316 d_{g 1}=7.14 \times 10^{-6}\right.$ $x_{2}=0.5684 d_{g 2}=1.63 \times 10^{-5} \mathrm{~cm}$ ) for $60 \%, 593^{\circ} \mathrm{C}, 1 \% \mathrm{H}_{2} \mathrm{~S}$ concentration 


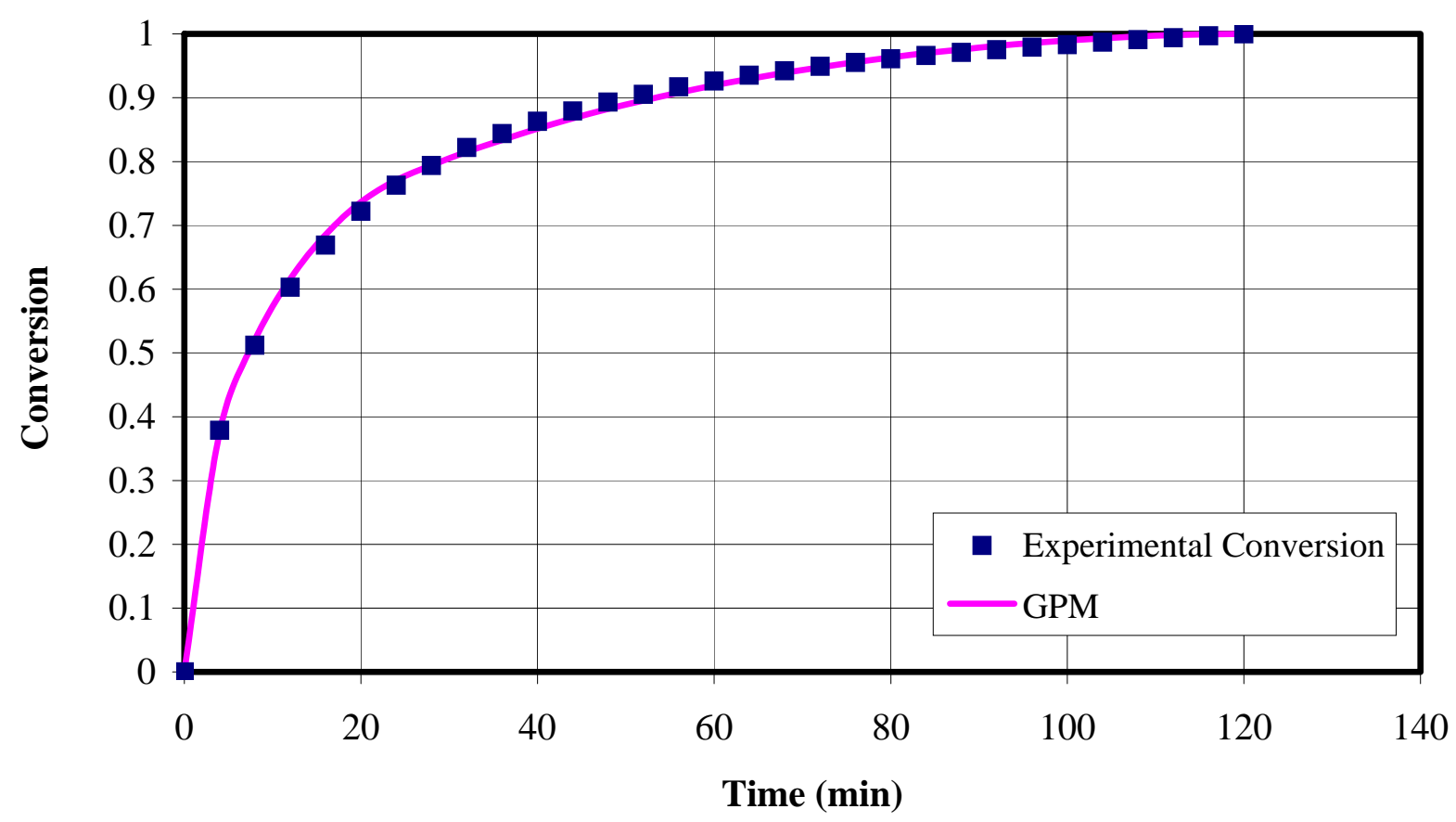

Figure 5.8: Experimental results compared with GPM using $Z_{v}\left(x_{1}=0.4316\right.$ $d_{g 1}=7.14 \times 10^{-6} x_{2}=0.5684 d_{g 2}=1.63 \times 10^{-5} \mathrm{~cm}$ ) for $60 \%, 593^{\circ} \mathrm{C}, 1 \% \mathrm{H}_{2} \mathrm{~S}$ concentration

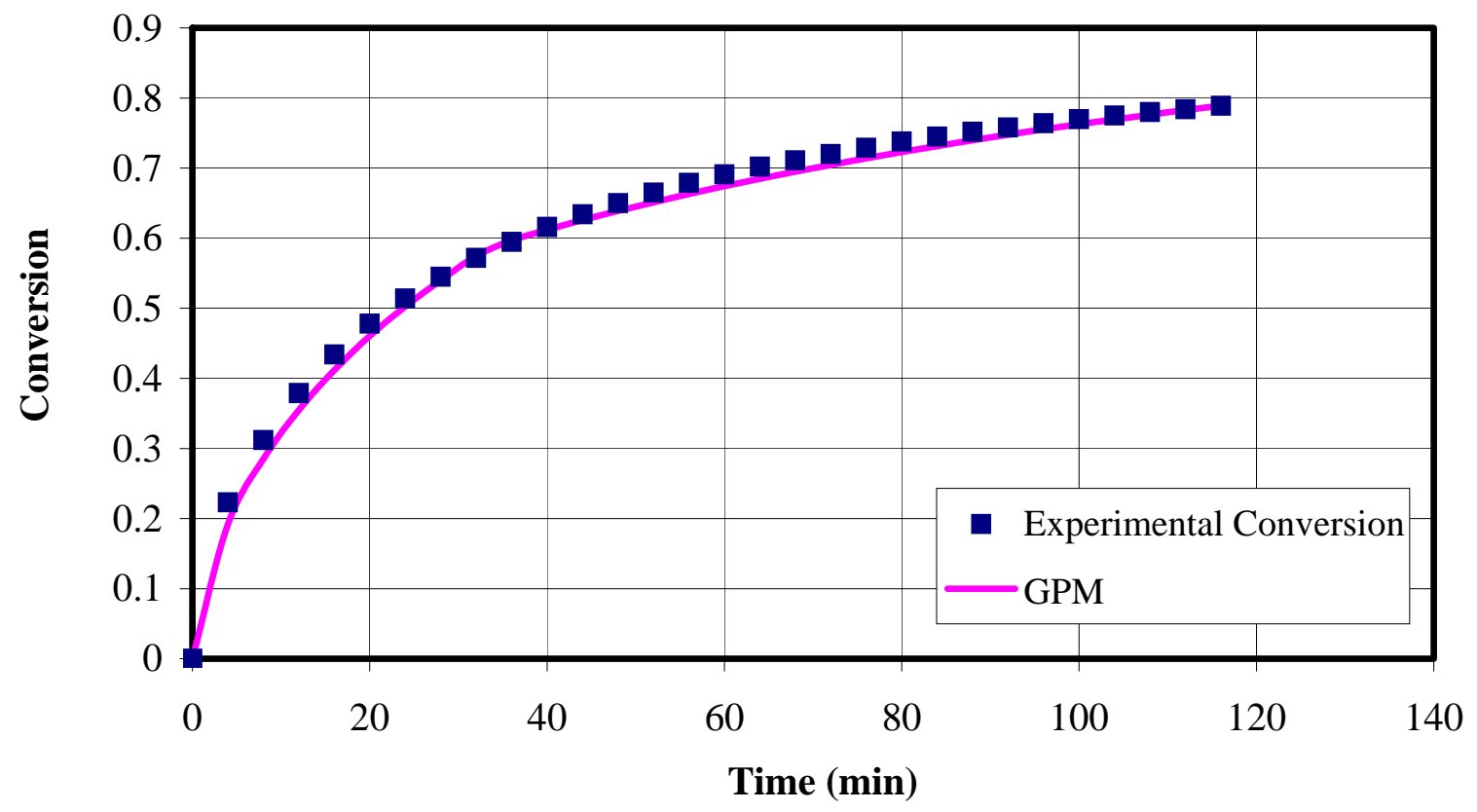

Figure 5.9: Experimental results compared with $G P M$ using $Z_{v}\left(x_{1}=0.4316\right.$ $d_{g 1}=7.14 \times 10^{-6} x_{2}=0.5684 d_{g 2}=1.63 \times 10^{-5} \mathrm{~cm}$ ) for $60 \%, 482^{\circ} \mathrm{C}$ and $1 \% \mathrm{H}_{2} \mathrm{~S}$ concentration 


\subsubsection{Activation Energy for Diffusion Coefficient $D_{e}$}

We assume that the effective diffusivity $D_{e}$ in the product layer obeys the following Arrhenius dependence on temperature:

$$
D_{e}=D_{o} e^{-E_{A} / R T}
$$

From Equation (49), we get for different temperatures:

$$
\begin{aligned}
& \frac{D_{e 1}}{D_{o}}=D_{o} e^{-E_{A} / R T_{1}} \\
& \frac{D_{e 2}}{D_{o}}=D_{o} e^{-E_{A} / R T_{2}}
\end{aligned}
$$

Dividing Equation (51) by Equation (52) and rewriting we get:

$$
\ln \left(\frac{D_{e 1}}{D_{e 2}}\right)=-\frac{E_{A}}{R}\left(\frac{1}{T_{1}}-\frac{1}{T_{2}}\right) \Longrightarrow E_{A}=\ln \left(\frac{D_{e 1}}{D_{e 2}}\right) \cdot R \cdot\left(\frac{1}{T_{2}}-\frac{1}{T_{1}}\right)
$$

For Figure 5.8 and 5.9, effective diffusivity $D_{e}$ for $593^{\circ} \mathrm{C}$ and $482^{\circ} \mathrm{C}$, are $1.07 \times 10^{-13}$ and $3.59 \times 10^{-14}\left(\mathrm{~m}^{2} / \mathrm{s}\right)$, respectively. When these values are substituted into Equation (53), we get:

$$
E_{A}=12.75 \quad(\mathrm{kcal} / \mathrm{mol})
$$

This value compares with $9.92 \mathrm{kcal} / \mathrm{mol}$, calculated using a generalized pore model by Efthimiadis and Sotirchos [6]; $22 \mathrm{kcal} / \mathrm{mol}$, calculated using a variable property grain model by Ranade and Harrison [4] and $26.4 \mathrm{kcal} / \mathrm{mol}$, calculated using an overlapping grain model by Lew et al. [11]. 


\subsubsection{Bimodal GPM for $80 \mathrm{wt} \%$ and $100 \mathrm{wt} \%$ Sorbents}

For $80 \%$ and $100 \%$ sorbents, we also use the bimodal grain size distribution to predict the conversion histories and compare with the results of the TGA experiments. Figure 5.10 and Figure 5.11 show the results for $80 \%$ sorbent while Figure 5.12 and Figure 5.13 give the results for $100 \%$ sorbent. 


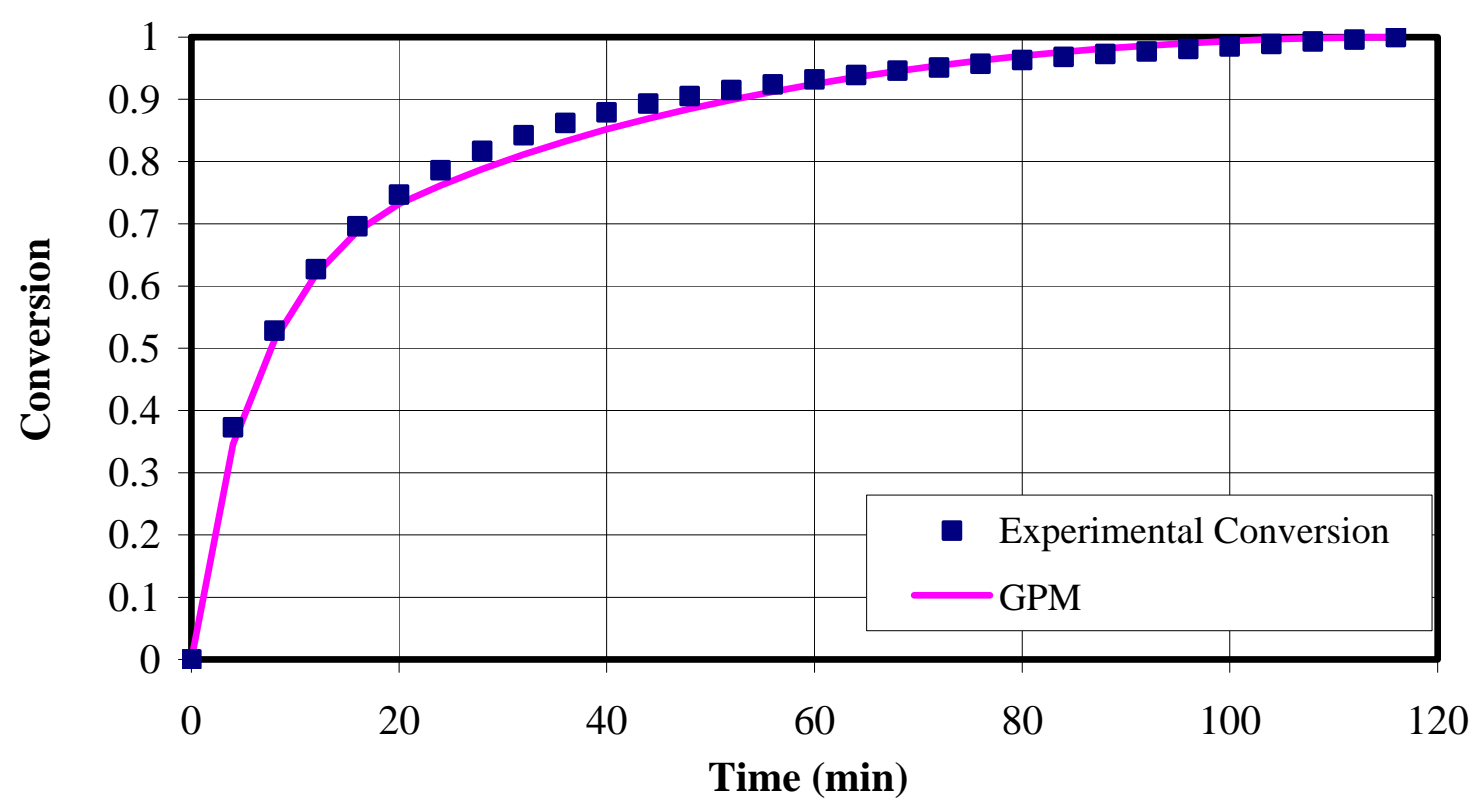

Figure 5.10: Experimental results compared with $\mathrm{GPM}$ using $\mathrm{Z}_{\mathrm{v}}\left(x_{1}=0.57, d_{g 1}=2.8 \times\right.$ $10^{-5}, x_{2}=0.43 d_{g 2}=9.54 \times 10^{-6} \mathrm{~cm}$ ) for $80 \%, 593^{0} \mathrm{C}, 1 \% \mathrm{H}_{2} \mathrm{~S}$ concentration

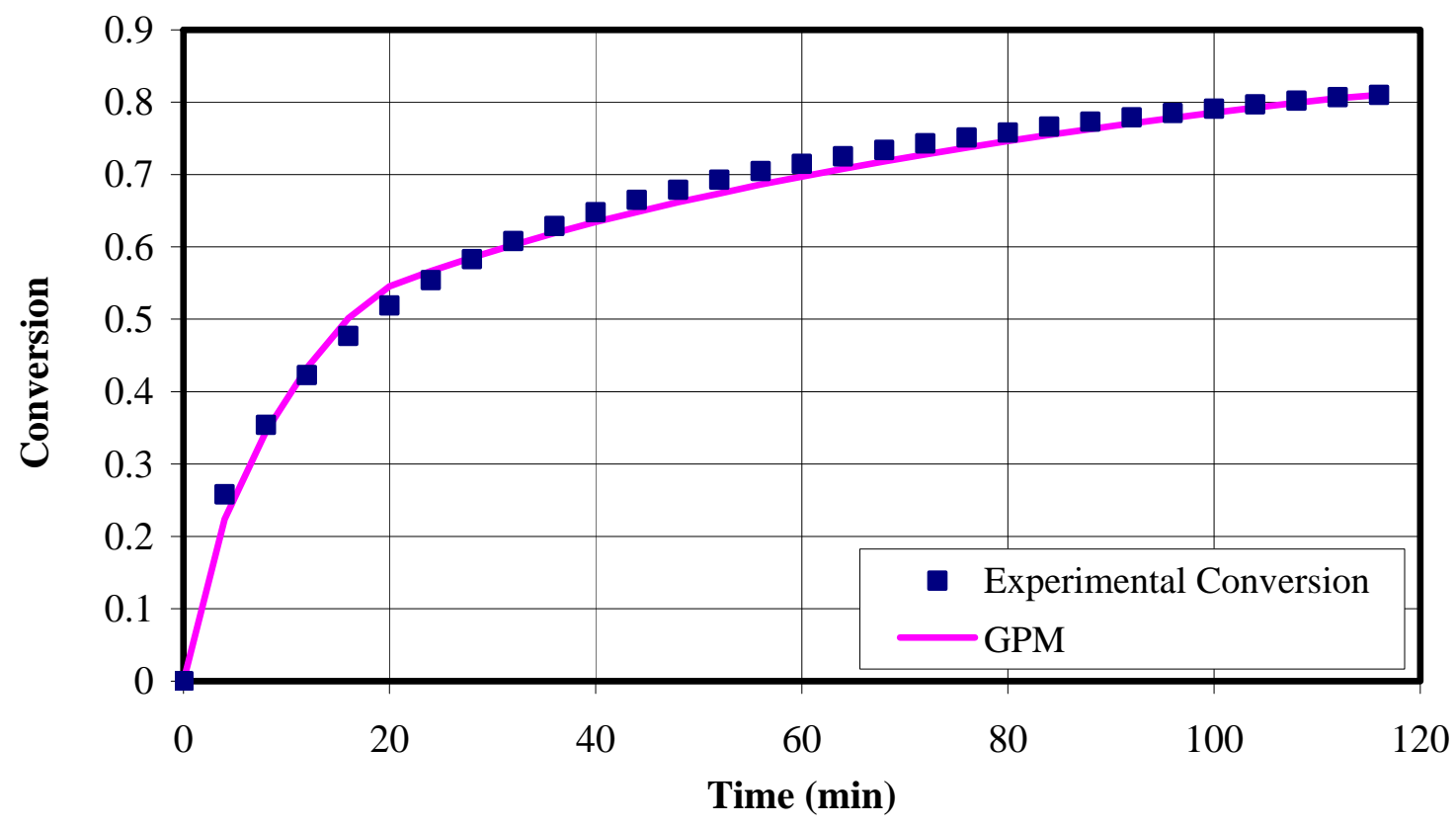

Figure 5.11: Experimental results compared with GPM using $\mathrm{Z}_{\mathrm{v}}\left(x_{1}=0.57\right.$

$d_{g 1}=2.8 \times 10^{-5} \quad x_{2}=0.43 \quad d_{g 2}=9.54 \times 10^{-6} \mathrm{~cm}$ ) for $80 \%, 482^{0} \mathrm{C}, 1 \% \mathrm{H}_{2} \mathrm{~S}$ concentration 


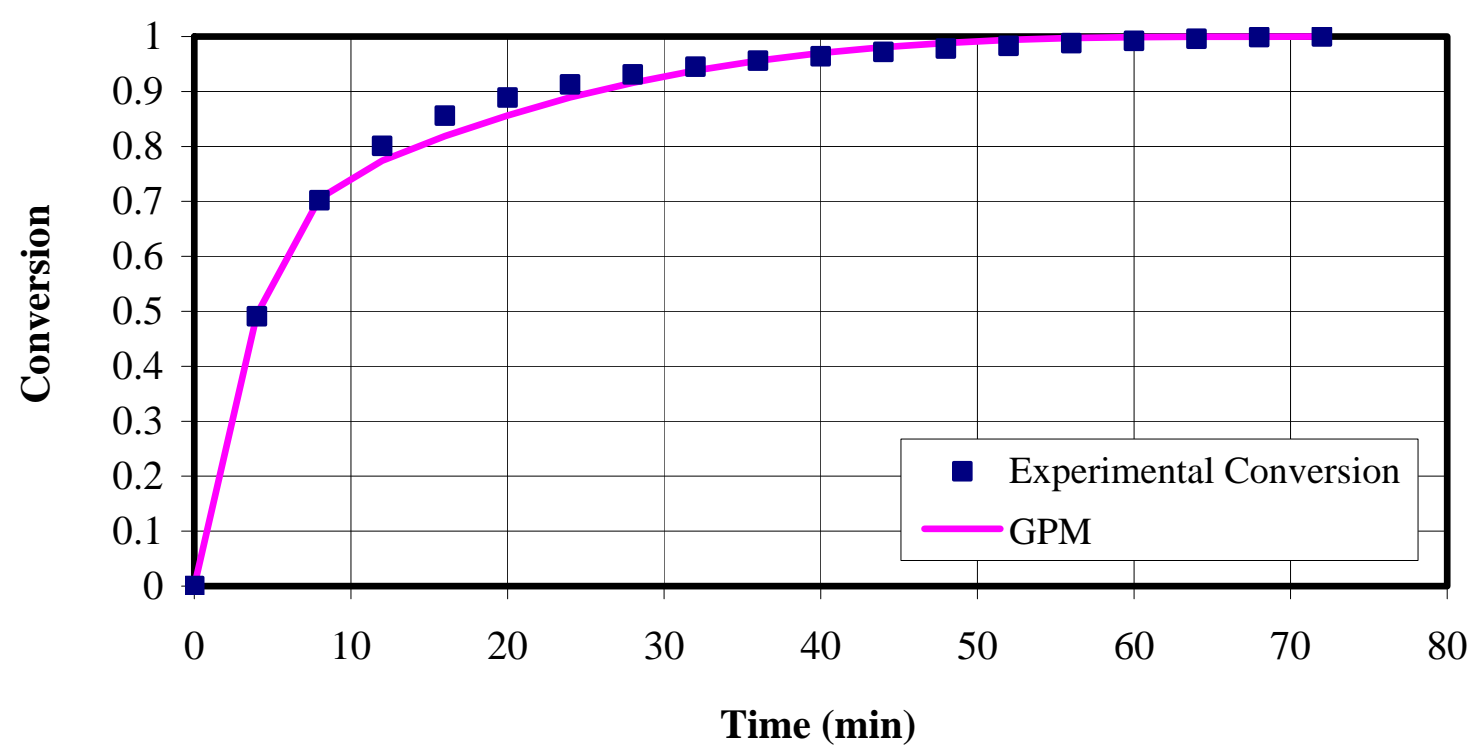

Figure 5.12: Experimental results compared with GPM using $\mathrm{Z}_{\mathrm{v}}\left(x_{1}=\mathbf{0 . 4}\right.$ $d_{g 1}=6.02 \times 10^{-5} x_{2}=0.6 d_{g 2}=1.02 \times 10^{-5} \mathrm{~cm}$ ) for $100 \%, 593^{\circ} \mathrm{C}, 1 \% \mathrm{H}_{2} \mathrm{~S}$ concentration

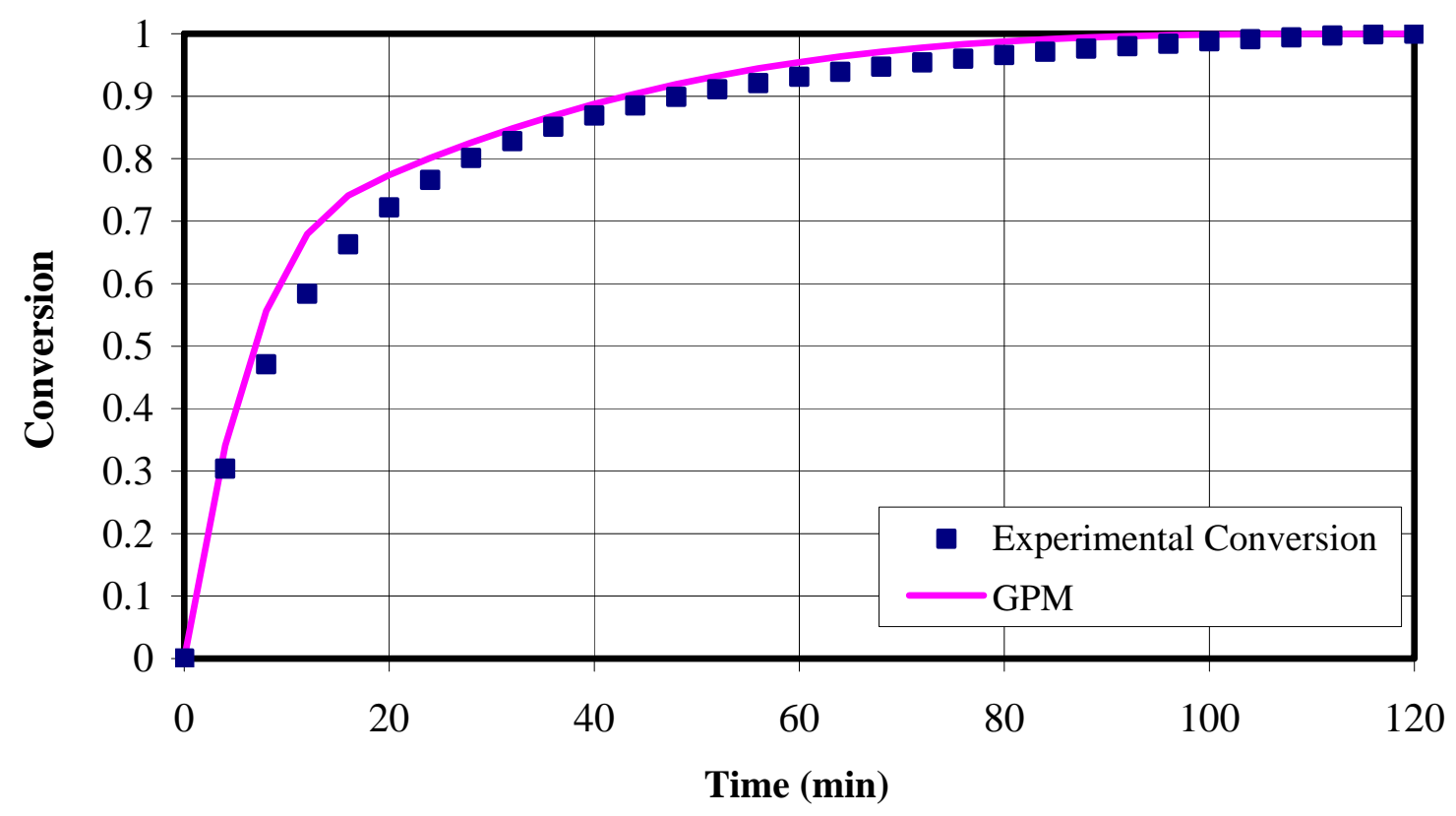

Figure 5.13: Experimental results compared with GPM using $Z_{\mathrm{v}}\left(x_{1}=0.4\right.$ $d_{g 1}=6.02 \times 10^{-5} x_{2}=0.6 d_{g 2}=1.02 \times 10^{-5} \mathrm{~cm}$ ) for $100 \%, 482^{\circ} \mathrm{C}, 1 \% \mathrm{H}_{2} \mathrm{~S}$ concentration 


\subsubsection{GPM Model using Ten Grain Sizes}

From the SEM image, it is clear that the sorbent is not just composed of two grain sizes. By using the Visilog ${ }^{\mathrm{TM}}$ software, we find the actual grain size distribution in the pellet, which is given below in Table 5.1.

Table 5.1: Grain size distribution

\begin{tabular}{|c|c|}
\hline Mass Fraction (100\%) & Diameter $(\mu \mathrm{m})$ \\
\hline 0.05 & 0.0704 \\
\hline 0.11 & 0.0707 \\
\hline 0.12 & 0.0660 \\
\hline 0.13 & 0.0619 \\
\hline 0.12 & 0.0602 \\
\hline 0.08 & 0.0587 \\
\hline 0.11 & 0.0588 \\
\hline 0.09 & 0.0582 \\
\hline 0.10 & 0.0581 \\
\hline 0.09 & 0.0557 \\
\hline
\end{tabular}

As with the two grain sizes used in Section 5.4.1, we can write the expressions for the conversion vs. time for each grain using Equation (45):

$$
t=\tau_{\text {ash }}\left[\frac{Z_{v}-\left[Z_{v}+\left(1-Z_{v}\right)(1-X)\right]^{2 / 3}}{Z_{v}-1}-(1-X)^{2 / 3}\right]+\tau_{\text {reac }}\left[1-(1-X)^{1 / 3}\right]
$$

Using Equation (26) for the ten grain sizes case we obtain the whole reaction history profile and then compare it with the experimental data. The result is given in Figure 5.14.

$$
\begin{aligned}
X= & 1-x_{1}\left(1-X_{1}\right)-x_{2}\left(1-X_{2}\right)-x_{3}\left(1-X_{3}\right)-x_{4}\left(1-X_{4}\right)-x_{5}\left(1-X_{5}\right) \\
& -x_{6}\left(1-X_{6}\right)-x_{7}\left(1-X_{7}\right)-x_{8}\left(1-X_{8}\right)-x_{9}\left(1-X_{9}\right)-x_{10}\left(1-X_{10}\right)
\end{aligned}
$$




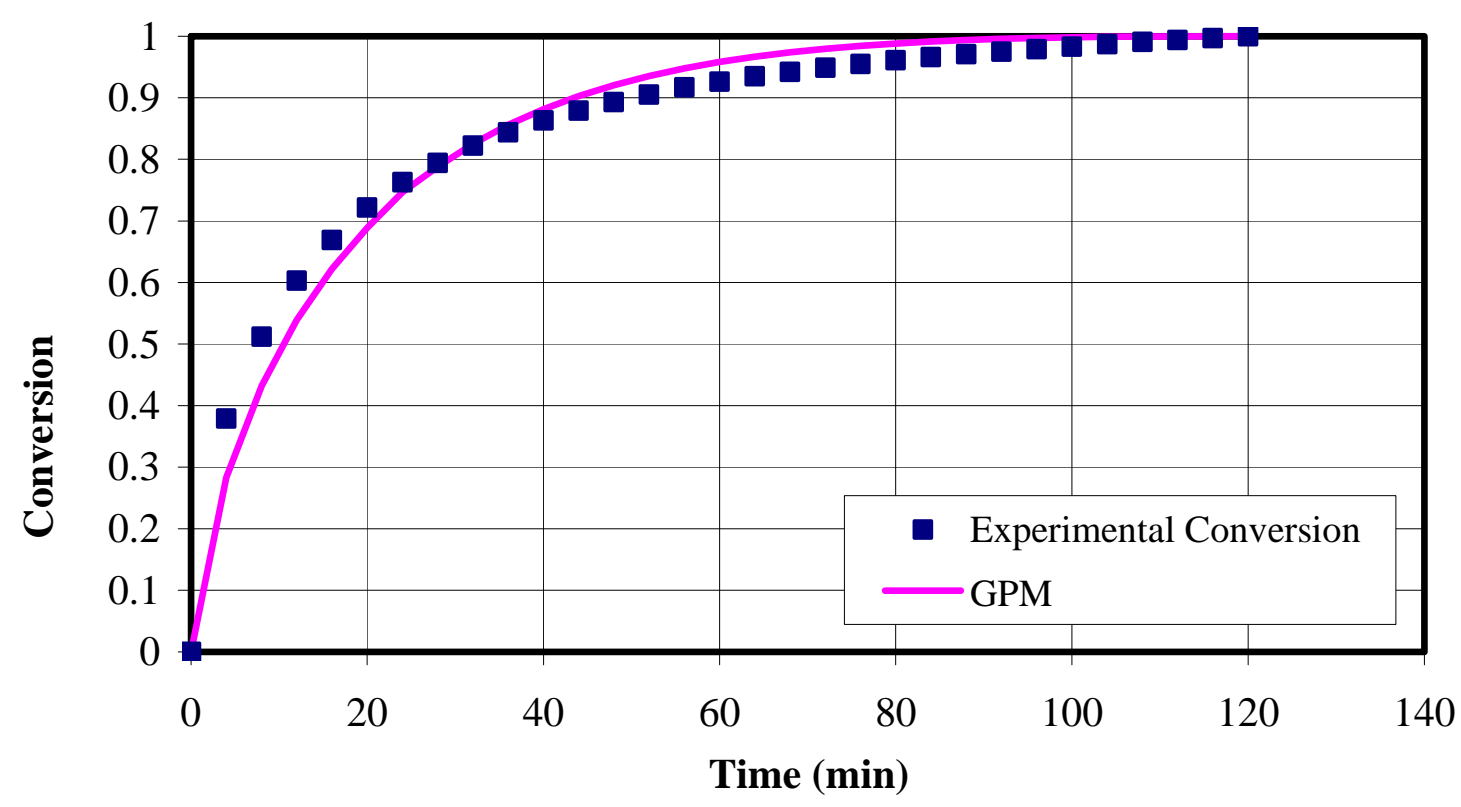

Figure 5.14: Experimental results compared with GPM (ten size distribution) for $60 \%, 593^{\circ} \mathrm{C}, 1 \% \mathrm{H}_{2} \mathrm{~S}$ concentration

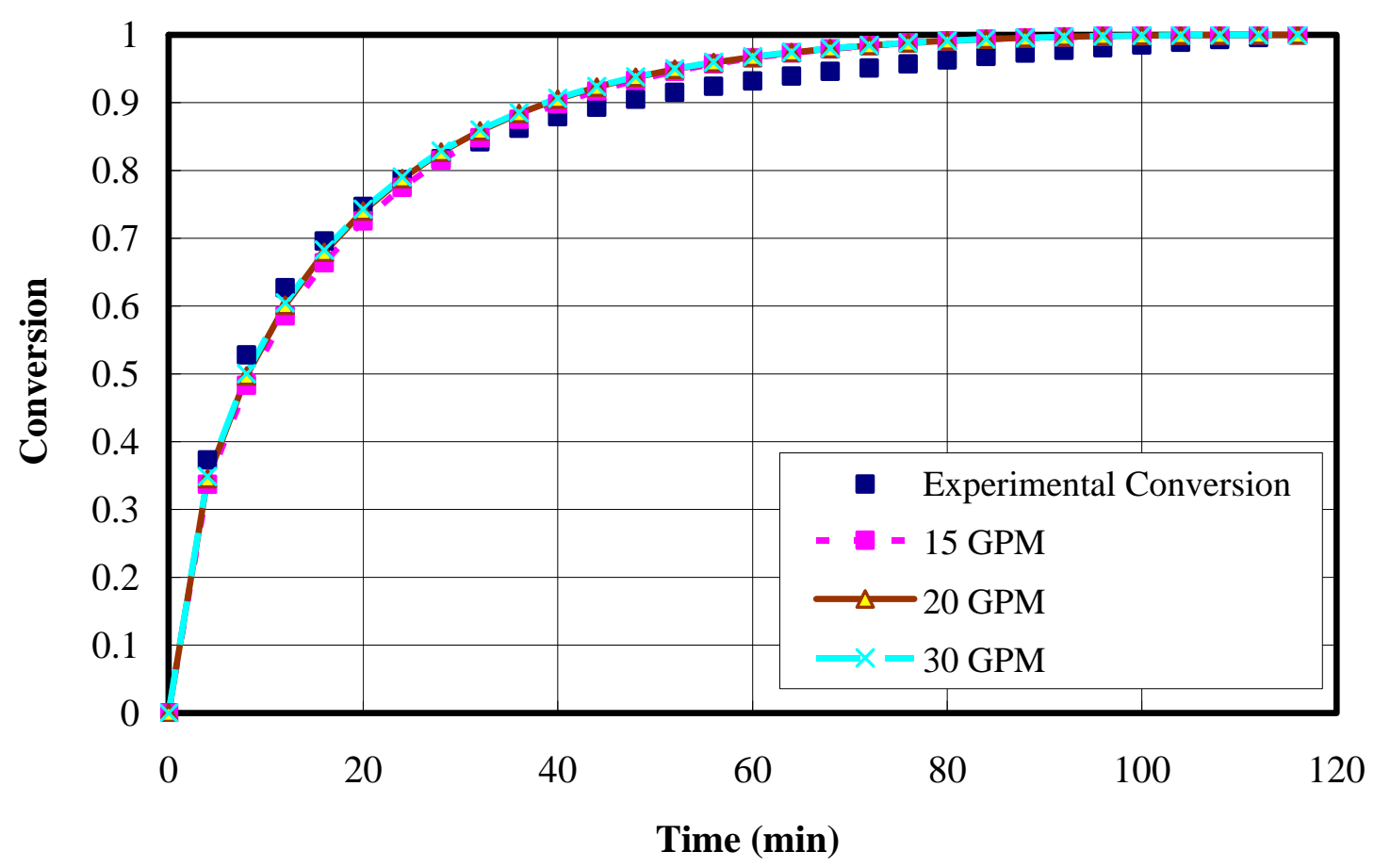

Figure 5.15: Experimental results compared with GPM (15/20/30 size distribution) for $80 \%, 593^{\circ} \mathrm{C}, 1 \% \mathrm{H}_{2} \mathrm{~S}$ concentration 


\subsubsection{GPM Model using 15/20/30 Grain Sizes}

GPM using 15, 20 and 30 grain sizes distribution were also examined. By comparing the experimental data and the model predictions, we can calculate the mean square error, which is shown in the following Table 5.2.

Table 5.2: Mean square error for GPM using 2, 10, 15, 20 and 30 grain sizes

\begin{tabular}{|c|c|}
\hline Number of Grain Sizes & Mean Square Error \\
\hline 2 & 0.073 \\
\hline 10 & 0.180 \\
\hline 15 & 0.138 \\
\hline 20 & 0.132 \\
\hline 30 & 0.131 \\
\hline
\end{tabular}

From the above table, it is clear that the bimodal is the best model to predict the experimental data. For GPM using 10,15 20 and 30 grain sizes in the distribution, 15 sizes gives a reasonable fit to the experimental conversion history. Figure 5.15 shows the case for the $80 \%$ sorbent.

\subsection{The Effect of Grain Shape}

\subsubsection{The Effect of Grain Shape on Conversion-Time History}

From the image of the sorbent, the shapes of grains appear to be non-spherical. In order to use equations derived for a spherical grain, we must find a shape factor, namely the sphericity, $\varphi$, to adjust the predictions of the model.

We define the sphericity in term of the image analysis data:

$$
\varphi=\frac{\text { Perimeter calculated from grain diameter obtained from image }}{\text { Perimeter from image analysis }}
$$


From the image analysis data, we obtain the area of the grain and by assuming the grain is spherical, we can derive the diameter of the grain. After we find the diameter of the grain, we can calculate the perimeter of the grain and compare it directly with that obtained from the image analysis. In the above analysis, it is assumed that the sphericities obtained from the projected area of the image is a representative shape factor.

The sphericities for the ten grain sizes given in Table 5.1 are shown in Table 5.3:

Table 5.3: Grain sphericity as a function of diameter

\begin{tabular}{|c|c|}
\hline Sphericity, $\varphi$ & Diameter $(\mu \mathrm{m})$ \\
\hline 0.656 & 0.0704 \\
\hline 0.527 & 0.0707 \\
\hline 0.422 & 0.0660 \\
\hline 0.352 & 0.0619 \\
\hline 0.311 & 0.0601 \\
\hline 0.280 & 0.0587 \\
\hline 0.262 & 0.0588 \\
\hline 0.244 & 0.0582 \\
\hline 0.231 & 0.0581 \\
\hline 0.211 & 0.0557 \\
\hline
\end{tabular}

The sphericity ranges from 0.21 to 0.66 , when $\varphi$ is close to 1 , the shape of grain is close to a sphere; when $\varphi$ close to 0 , the shape of grain is close to a disc.

In order to include the sphericity in the model, we substitute $\varphi \cdot R$ for $R$ in Equation (45) and recalculate the conversion-time history. This is shown in Figure 5.16.

From the figure, it can be seen that this modification to the grain model degrades the prediction. 


\subsubsection{Effect of Different Shaped Grains on Conversion-Time History}

As the sphericity of the grain gets lower, the application of equations based on a spherical grain become less appropriate, and the shape of the grains approach a disc or flat plate.

The equation for flat-plate shaped grains, the GPM is, (see Appendix)

$$
\begin{aligned}
& t=\tau_{\text {ash }} X^{2}+\tau_{\text {reac }} X \\
& \tau_{\text {ash }}=\frac{\rho_{\mathrm{ZnO}} L^{2} Z_{v}}{2 D_{e} C_{A b} M_{Z n O}} \quad \tau_{\text {reac }}=\frac{\rho_{\mathrm{ZnO}} L}{k C_{A b} M_{\mathrm{ZnO}}}
\end{aligned}
$$

Where, $L$ is the half thickness of the plate.

By assuming all grains with $\varphi>0.5$ are spherical and all $\varphi<0.5$ are flat-plate, the conversion-time history is given by Figure 5.17. From the result, it is clear that this modification is also not successful. 


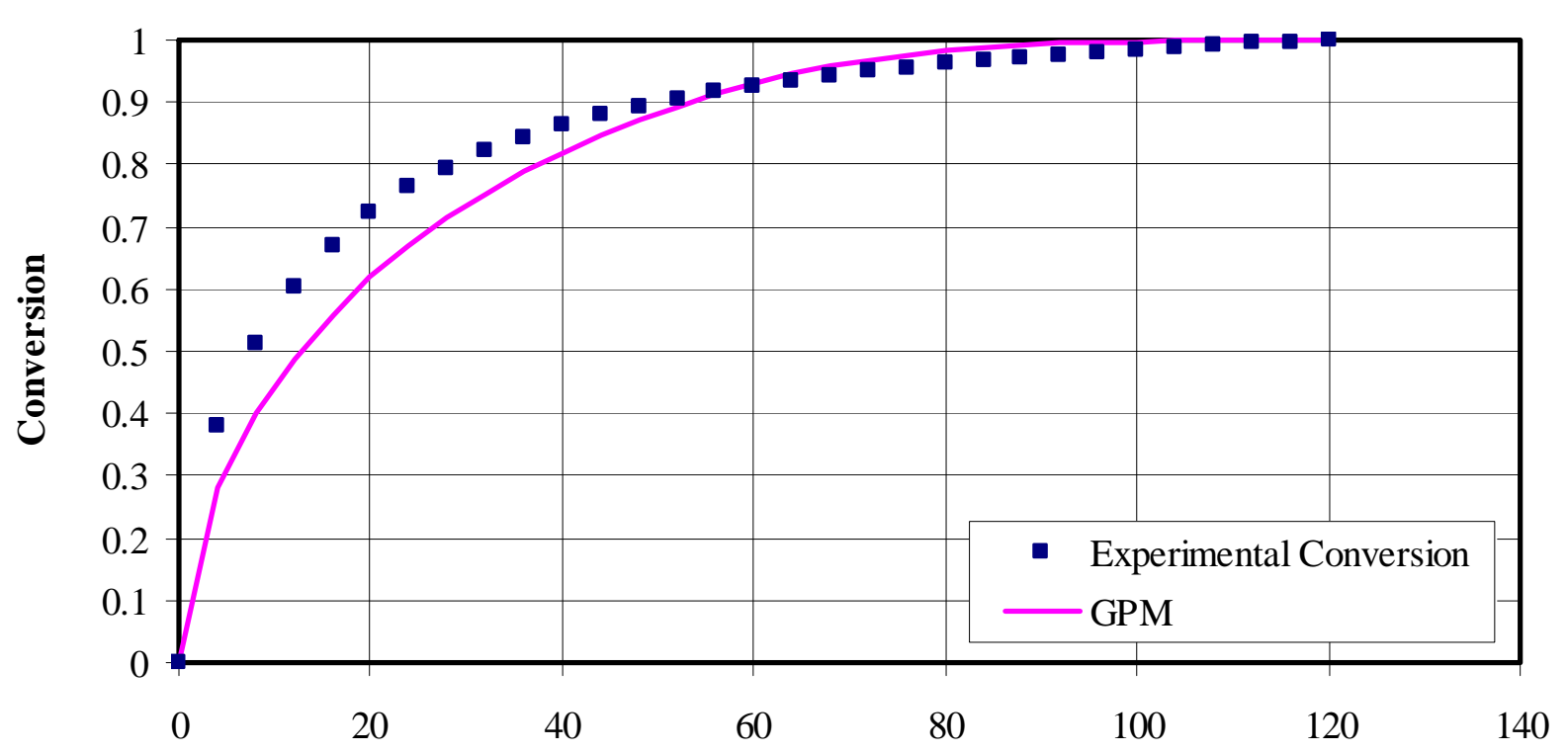

Time (min)

Figure 5.16: Experimental results compared with GPM using sphericity (ten size distribution) for $60 \%, 593^{\circ} \mathrm{C}, 1 \% \mathrm{H}_{2} \mathrm{~S}$ concentration

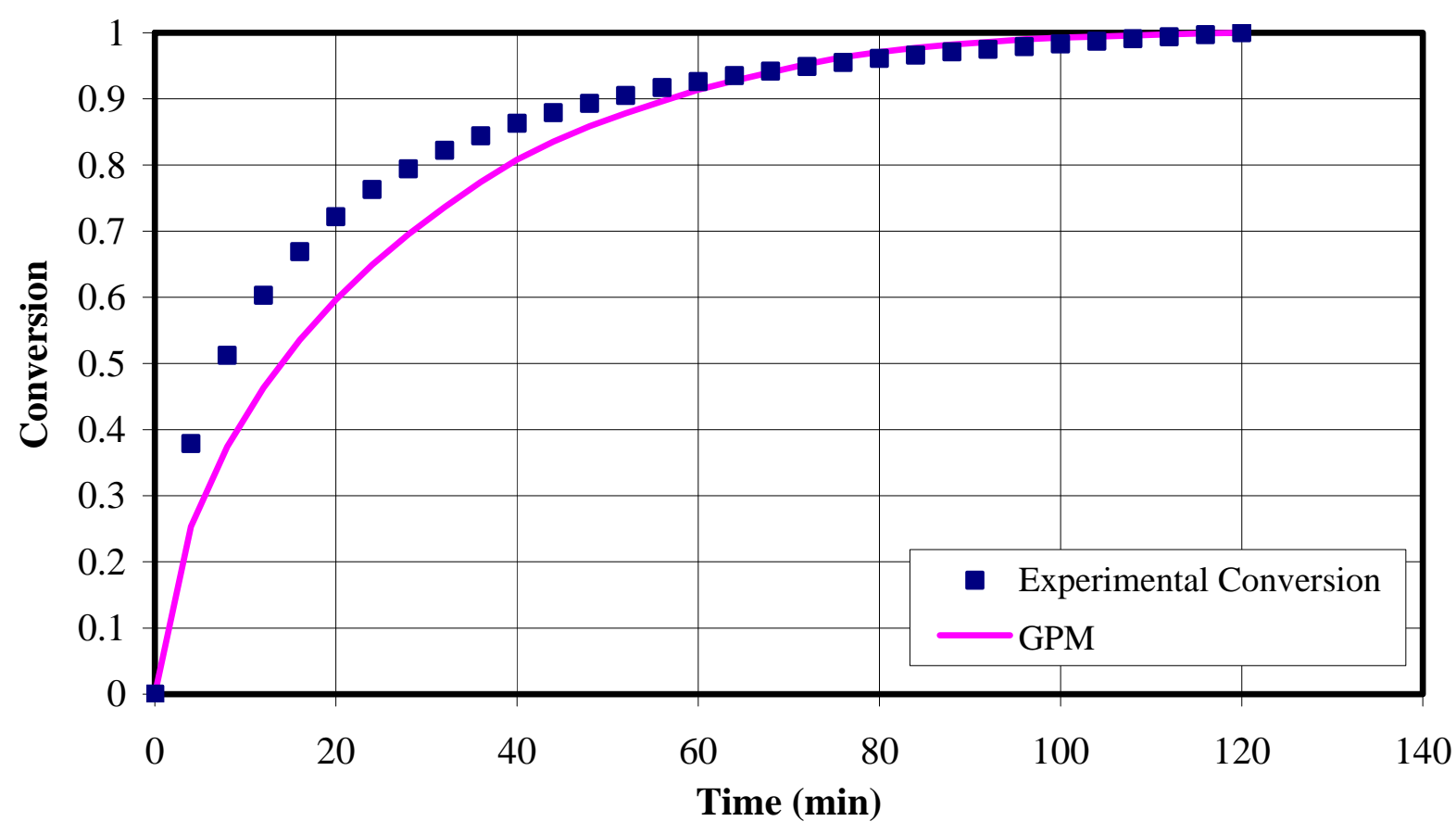

Figure 5.17: Experimental results compared with ten size GPM (spherical and plate) for $60 \%, 593^{\circ} \mathrm{C}, 1 \% \mathrm{H}_{2} \mathrm{~S}$ concentration 


\subsubsection{Effect of using flat plate-shaped GPM}

Since the biggest grain sphericity among the ten grains is 0.656 , we might conclude that the shape of all ten grains is more close to a disc than to a sphere. Therefore, we may use the flat plate-shaped GPM to predict the reaction profile. The result is shown in Figure 5.18. From the figure, it is clear that this modification is also not successful.

\subsubsection{Ten Size Distribution GPM for 80 wt \% Sorbents}

For the $80 \%$ sorbents, when using ten grain sizes GPM to predict the conversion profiles and compare with the results of the TGA experiments, we get similar results as for the $60 \%$ sorbent case; namely, the basic ten sphere-shaped GPM is the best one if we consider grain shape factor. Figure 5.19 shows the 10 grain sizes GPM prediction compares with the experimental data for $80 \%$ sorbent.

From above, we might conclude that if we consider the shape factor in our model, the modification degrades the prediction. The reason for this result is that we have introduced an artifact vision of the system by using sphericity. Namely, the large grains measured by the Visilog ${ }^{\mathrm{TM}}$ are comprised of many small particles. The software calculates sphericity for this combined mass when in reality the sphericity and particle size should be for the individual grains. However, the contrast in the SEM is not sharp enough for the software to identify the individual grains. 


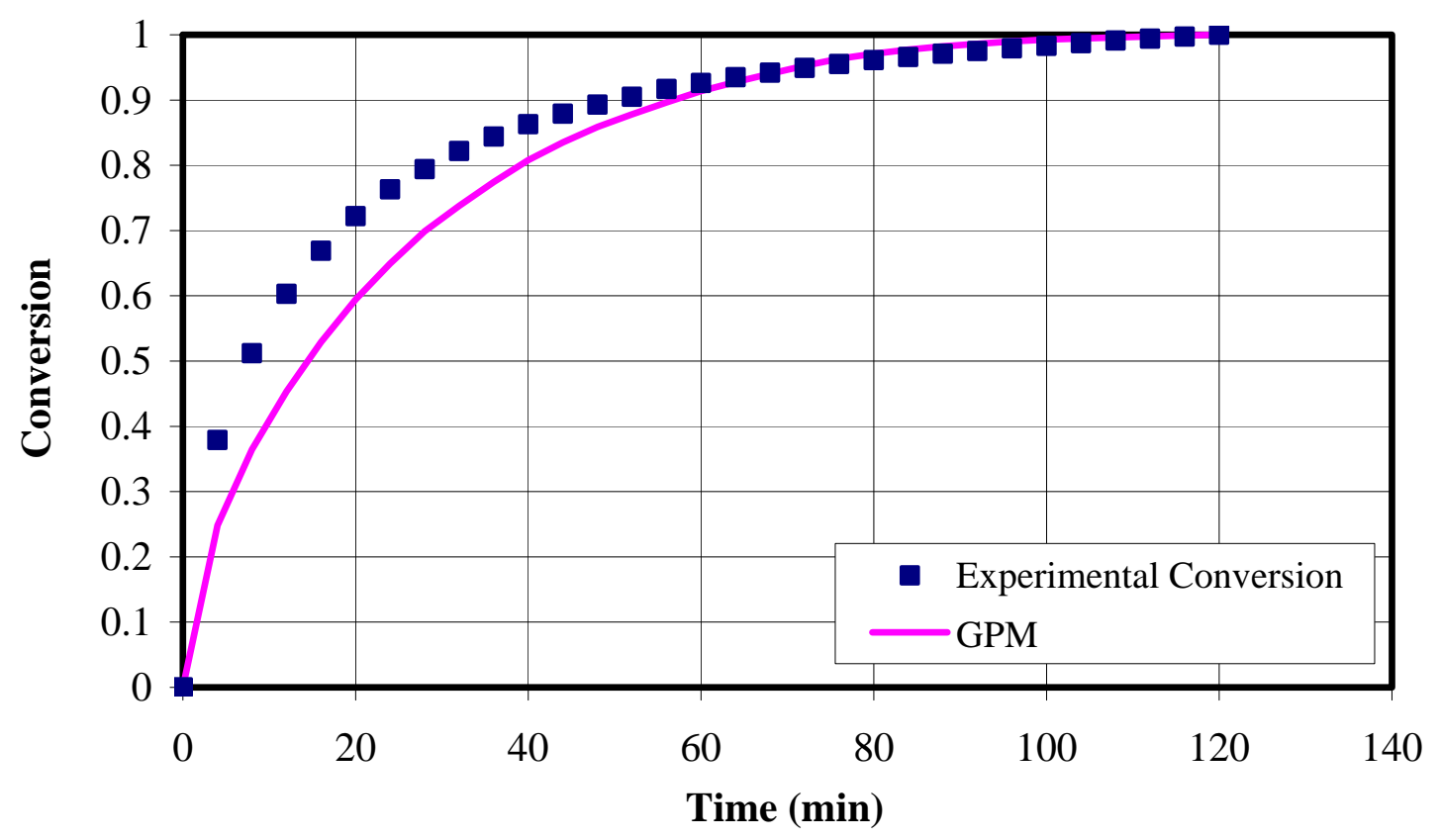

Figure 5.18: Experimental results compared with flat-plate shaped using 10 grain sizes GPM for $60 \%, 593^{\circ} \mathrm{C}, 1 \% \mathrm{H}_{2} \mathrm{~S}$ concentration

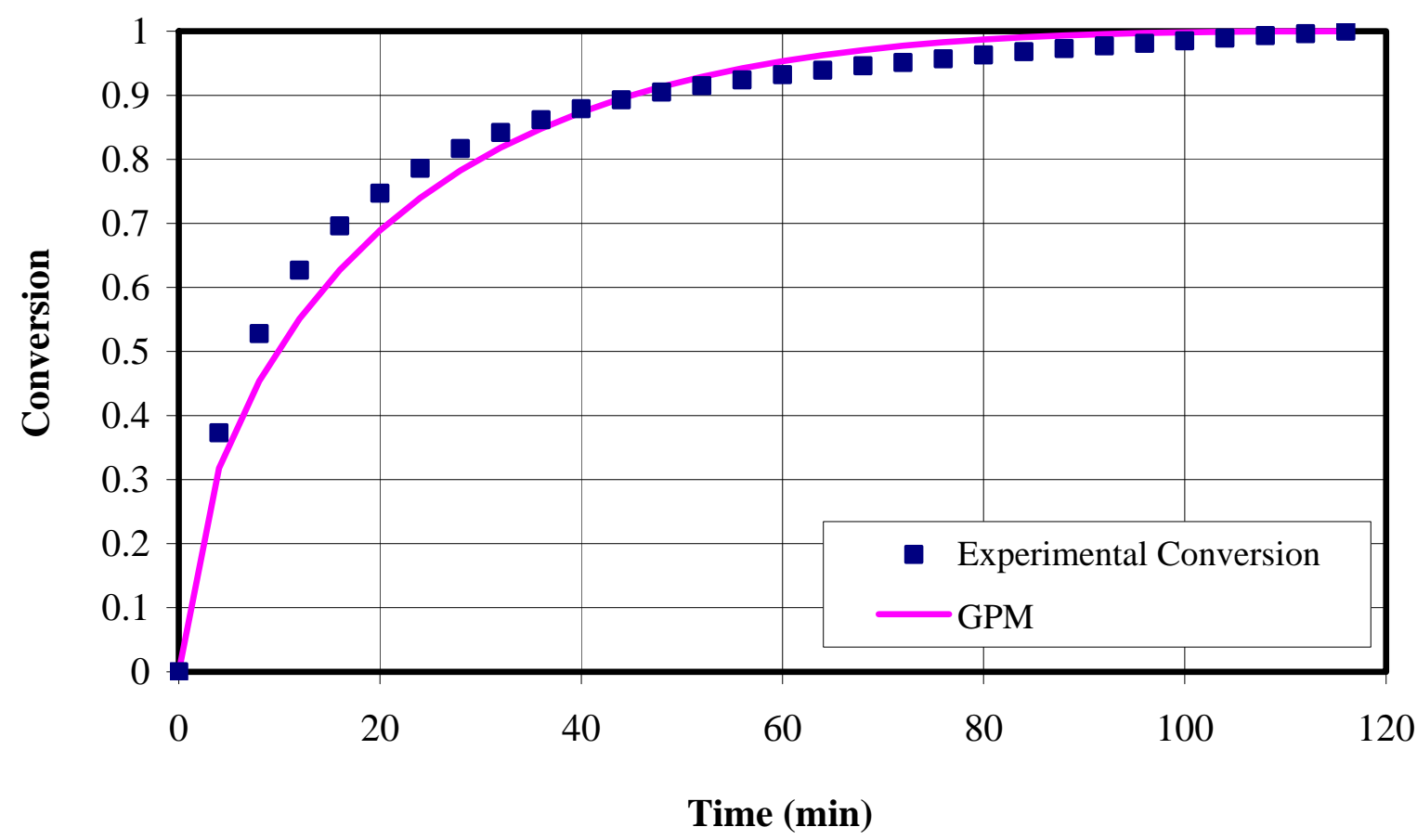

Figure 5.19: Experimental results compared with GPM (ten grain size distribution) for $80 \%, 593^{\circ} \mathrm{C}, 1 \% \mathrm{H}_{2} \mathrm{~S}$ concentration 


\subsection{Images From Scanning Electron Microscope (SEM)}

\subsubsection{SEM Images}

From SEM, we can get a better understanding of how the zinc oxide is distributed in the alumina support. Fresh and reacted sorbents (60\%, $80 \%$ mass fraction of $\mathrm{ZnO})$ were analyzed using a SEM model S-4700 (Instruments, Hitachi, Ltd.) equipped with a X-ray microanalysis instrument (EDAX Inc, NJ).

Figure 5.20 is an SEM image at $30 \times$ time magnification of the fresh $80 \%$ sorbent. Every circle is a zinc oxide particle which is distributed in epoxy.

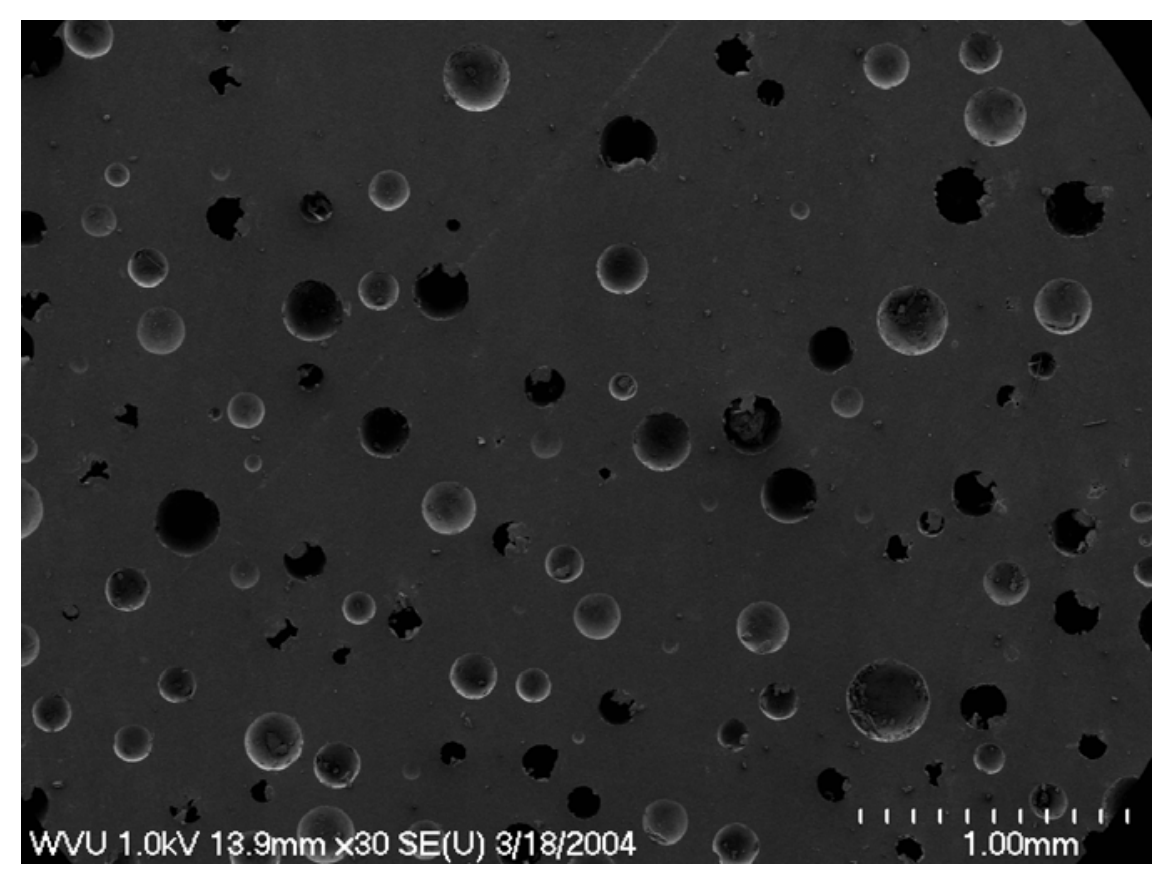

Figure 5.20: SEM for fresh $80 \%$ sorbent of 30 time magnification

Figure 5.21 and Figure 5.22 are captured from different locations within a fresh $80 \%$ sorbent zinc oxide particle, while Figure 5.23 and 5.24 are for reacted $80 \%$ sorbent. 


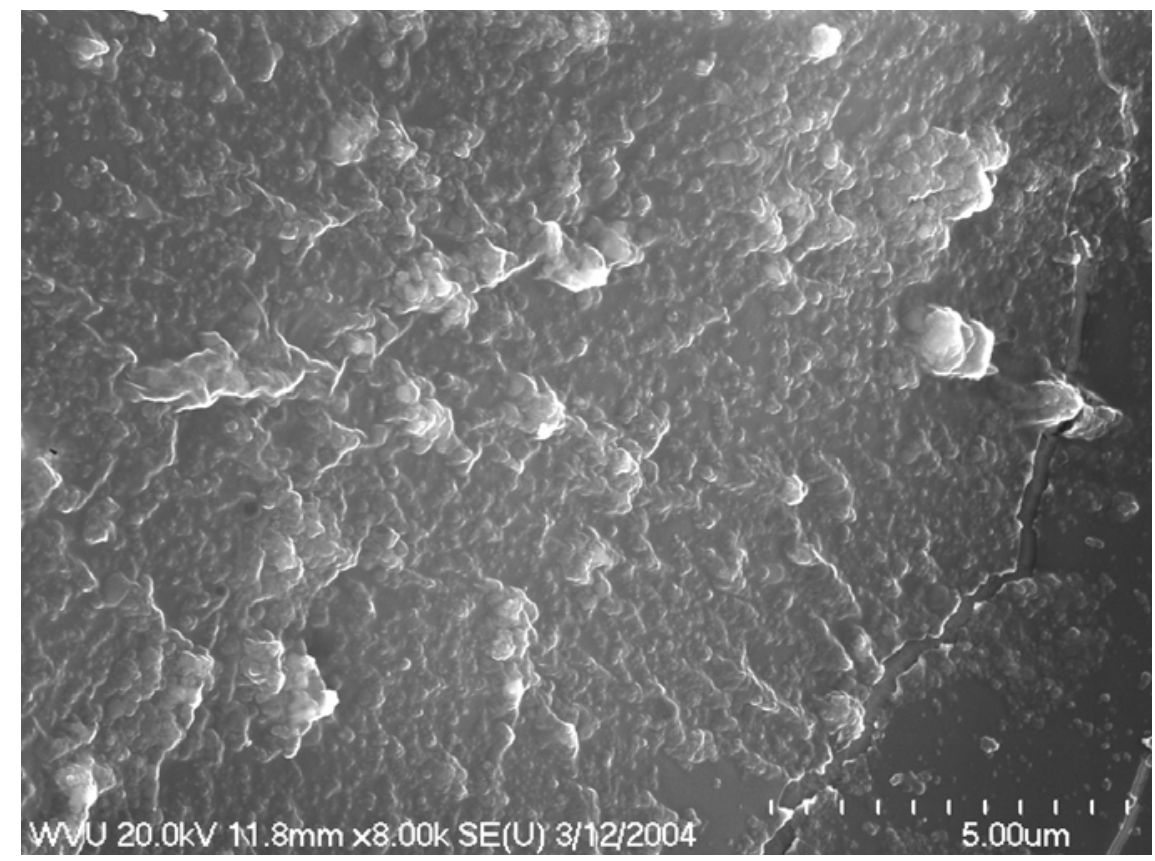

Figure 5.21: SEM for fresh $80 \%$ sorbent using $8 \mathrm{~K}$ magnification

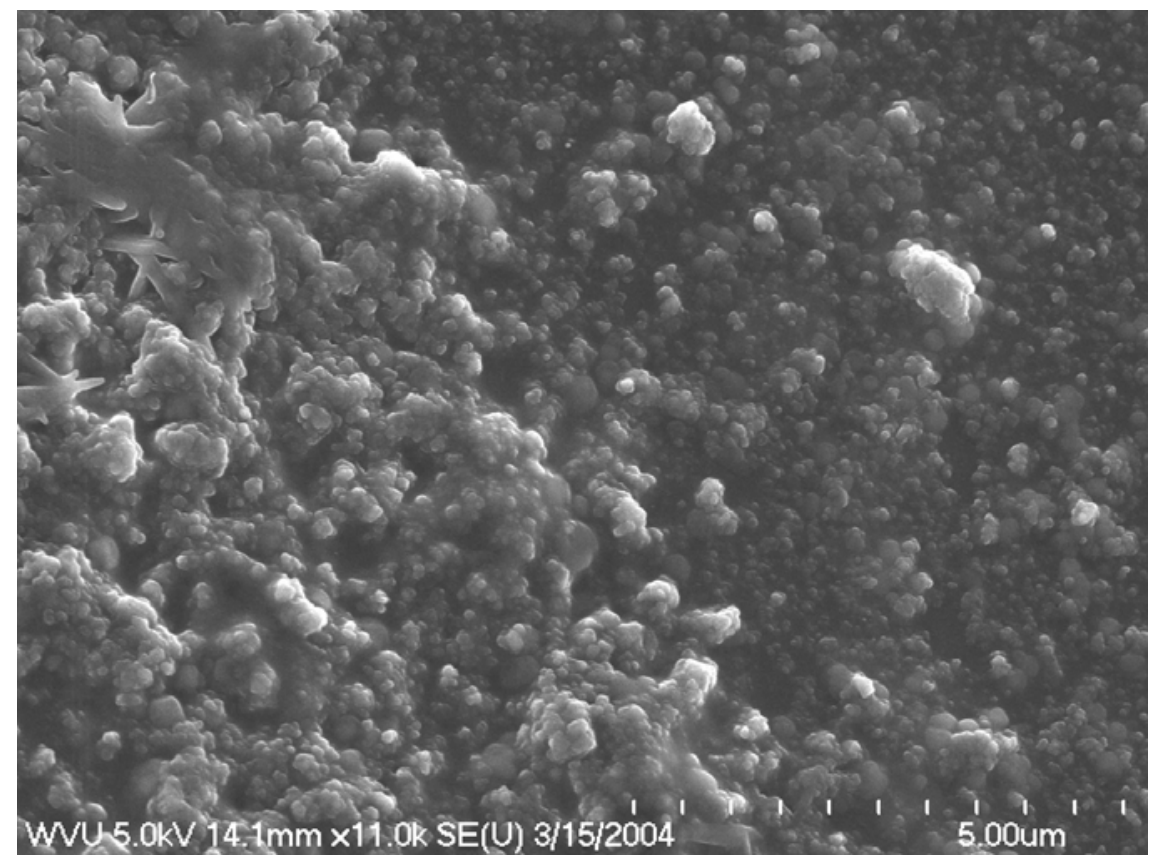

Figure 5.22: SEM for fresh $80 \%$ sorbent using $11 \mathrm{~K}$ magnification 


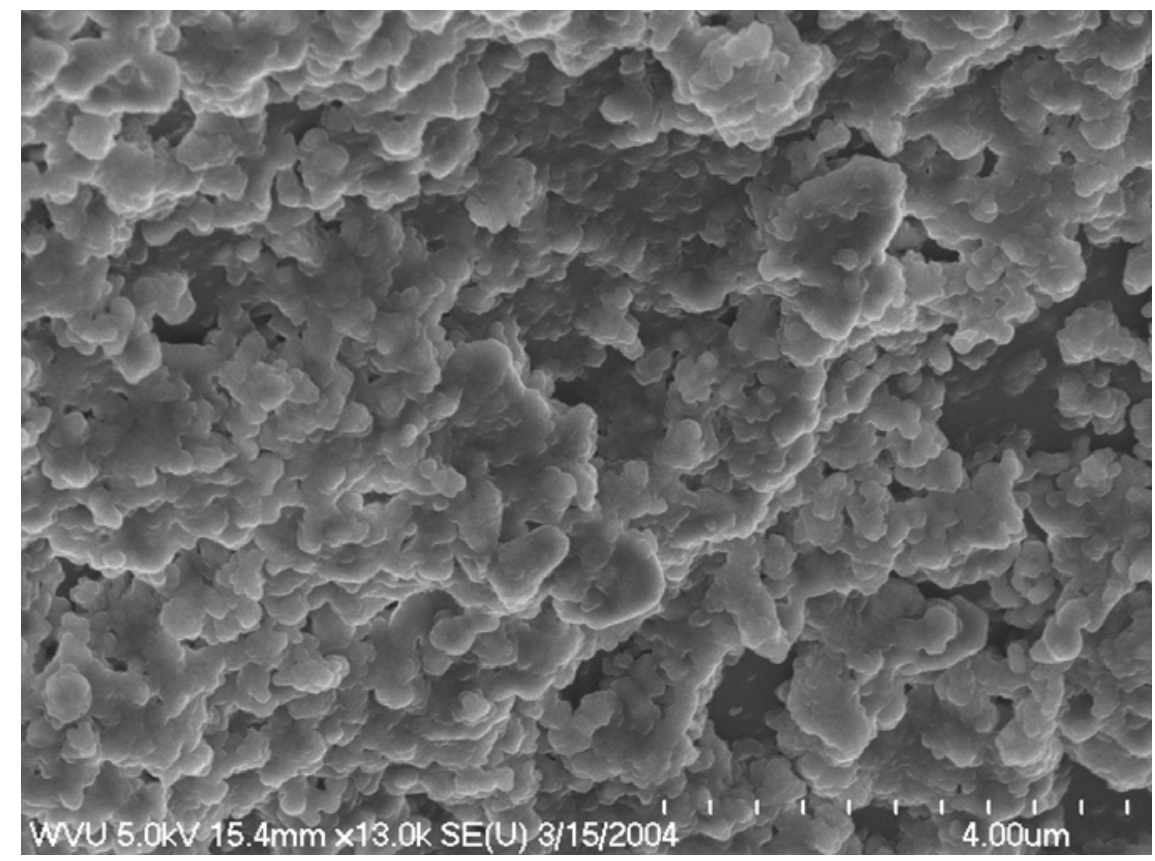

Figure 5.23: SEM for reacted $80 \%$ sorbent using $13 \mathrm{~K}$ magnification

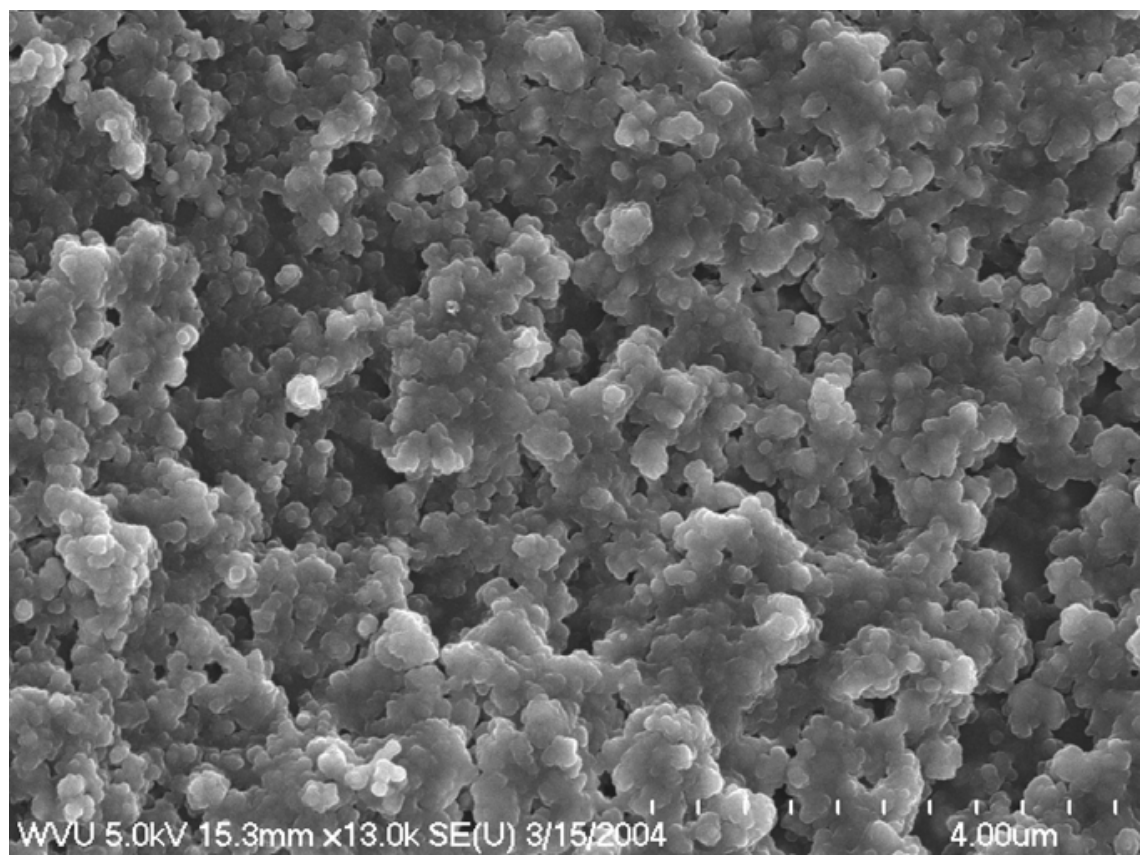

Figure 5.24: SEM for reacted $80 \%$ sorbent using $13 \mathrm{~K}$ magnification 
As was mentioned previously, because the solid product $(\mathrm{ZnS})$ occupies more volume than a stoichiometrically equivalent amount of solid reactant $(\mathrm{ZnO})$, the grains shown in the SEM image for the partially reacted sorbent connect/overlap each other. While for the fresh sorbent, the SEM image shows quite a different pattern.

\subsubsection{X-ray Microanalysis Results}

By using X-ray microanalysis, it was hoped that we could obtain a chemical analysis of the sorbents and would, therefore, be able to evaluate the distribution of zinc and alumina within the sorbent. However, from the following Figures (Figures 5.26 and 5.27), it is clear that there appears to be no way to distinguish between the two elements, since the zinc dots and alumina dots are present through out the SEM image. The reasons for this result maybe due to one or more of the following: when preparing the sample, the polishing machine smears the alumina and zinc oxide together; when using X-ray, the voltage need is between $20 \mathrm{KV}$ and $30 \mathrm{KV}$, such an intense beam penetrates several microns into the solid and thus an average reading over this depth is obtained. Thus the signal is not a true surface scan and the zinc and alumina appear to be smeared.

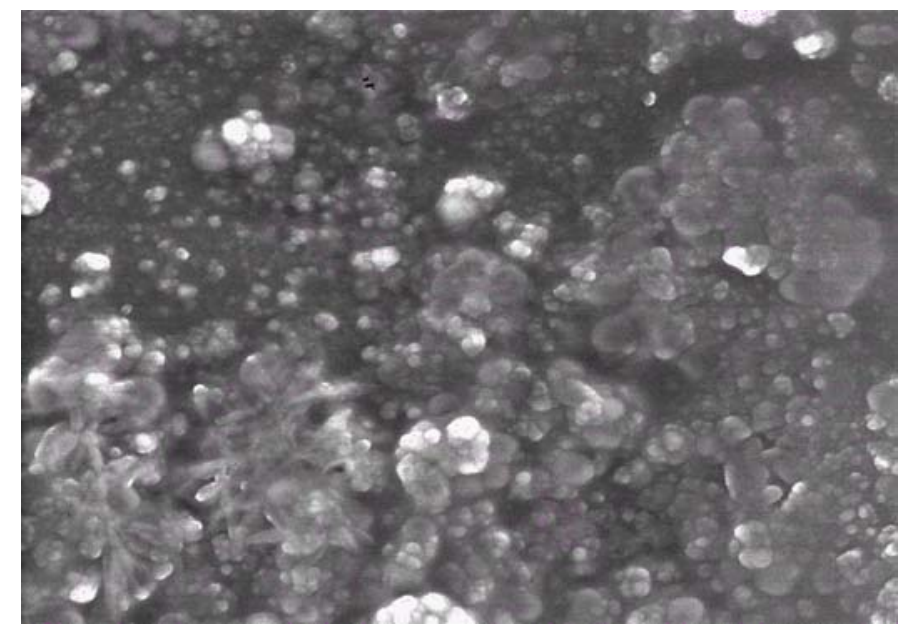

Figure 5.25: Overlay X-ray (Zn \& Al) for fresh $80 \%$ sorbent 
Figure 5.26: $\mathrm{X}$-ray for aluminum for fresh $\mathbf{8 0} \%$ sorbent

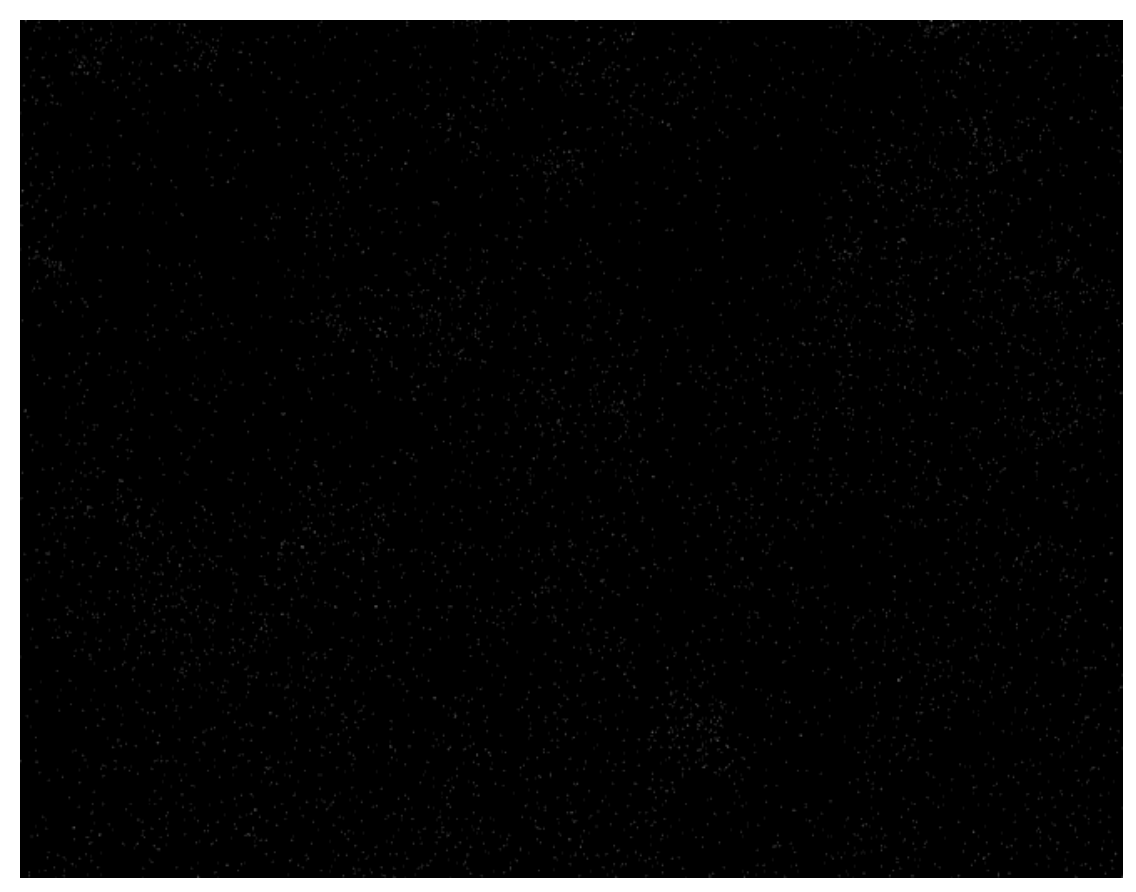

Figure 5.27: X-ray for zinc for fresh $80 \%$ sorbent 


\subsection{Overlapping Grain Model (OGM)}

Both the SCM and GPM use populations of nonoverlapping grains of uniform size to predict reactivity and surface area evolution data for gas-solid reactions. But from the SEM images, it is clear that the grains are not isolated from each other but rather they overlap. Therefore, the OGM may give a more reasonable description of the actual reaction profile.

Sotirchos and Yu [16] developed the OGM based on the assumption that the pore and reaction surfaces of the reacting solid may be represented by two populations of overlapping grains which share the same centers (spherical grains), axes (cylindrical grains), or planes (platelike grains) of symmetry.

They defined the porosity of the sorbent $\varepsilon_{r}$ as:

$$
\varepsilon_{r}=\exp \left[-n \int_{Y(t)}^{r_{0, \max }} r^{m} n_{o}\left(r_{o}\right) d r_{o}\right]
$$

where, $n$ is the grain geometric factor (sphere $=4 \pi / 3$; cylinder $=\pi / L_{\text {ave }}$; plate $=2 A_{\text {ave }}$ ); $m$ is the grain shape factor (sphere=3; cylinder=2; plate=1); $L_{\text {ave }}$ and $A_{\text {ave }}$ are the average grain length of the cylinder and the average grain surface area of plate-like grains, respectively; $r$ is the grain radius of initial size $r_{o}$ at the reaction interface; $r_{o}$ is the initial grain radius; $r_{o, \max }$ is the initial maximum grain radius; $n_{o}\left(r_{o}\right) d r_{o}$ is the number of grains per unit volume with radius in the initial size range $\left[r_{o}, r_{o}+d r_{o}\right]$ and $Y(t)$ is the lower active reactant grain radius limit of the initial size range from which grains of the reaction surface of non-zero size originate.

An equation for the change of $Y(t)$ is derived by noting that $r(Y(t), t)=0$ and so through 
differentiating, we get

$$
\frac{d Y(t)}{d t}=-\left[\frac{\left(\frac{\partial r}{\partial t}\right)}{\left(\frac{\partial r}{\partial r_{o}}\right)}\right]_{r_{o}=Y(t)}
$$

The porosity of the solid (reactant product) is found from

$$
\varepsilon_{p}=\varepsilon_{o}-\left(Z_{v}-1\right)\left(\varepsilon_{r}-\varepsilon_{o}\right)
$$

where $\varepsilon_{0}$ is the initial porosity of solids.

The grain radius $r$ is related to time by:

$$
\frac{d r}{d t}=\frac{\frac{k C_{A b} M_{B}}{\rho_{B}}}{1-\frac{k}{D_{e}} \varepsilon_{r} r^{m-1} \int_{r_{p}}^{r_{r}} \frac{d r}{\varepsilon\left(t^{\prime}\right) r^{m-1}}}
$$

where $r_{p}$ is the grain radius at the pore surface (solid reactant + product), $\varepsilon$ is the solids porosity, at $r_{p}, \varepsilon=\varepsilon_{p}$ and at $r_{r}, \varepsilon=\varepsilon_{r}$. The change in the pore radius $r_{p}$ is related to the reaction surface $r$ by

$$
\frac{d r_{p}}{d t}=-\frac{d r_{r}}{d t}\left[\left(Z_{v}-1\right) \frac{\varepsilon_{r} r^{m-1}}{\varepsilon_{p} r_{p}^{m-1}}\right]
$$

And, the fractional conversion is calculated as

$$
X=\frac{\varepsilon_{o}-\varepsilon_{p}}{\left(Z_{v}-1\right)\left(1-\varepsilon_{o}\right)}
$$


The computational procedure consists of three general steps: (a) use of the discretized form of Equation (56) to get the lower active reactant grain radius limit, (b) discretize Equation (57) and (58) with $\varepsilon_{p}$ and $\varepsilon_{r}$ given by Equation (27) and (28), (c) use of Equation (29) to compute model fractional conversion at selected times.

This model seems to be more consistent with the actual situation, thus is significantly more complicated and involves many calculations compared with our bimodal GPM which is the best match with the experimental data. Because of the complexity of this model, it was not tested in this work. 


\section{CONCLUSIONS}

The basic forms of the SCM and GPM models could not predict correctly the observed conversion-time data for pure zinc oxide sulfidation in this study. The only unknown parameter, $D_{e}$, is also fixed by the total reaction time for these two models under experimental conditions. Therefore, variations of these models were used to explain the experimental results.

Modifications to the models included considering: the effect of conversion on the physical properties of the sorbent due to the difference in molecular volume of zinc oxide and zinc sulfide, the distribution of grains size, and differences in grain shape.

Of these models, a bimodal GPM gave the best match with the experimental data. The bimodal GPM uses the total reaction surface area (computed from Equation (36)) to back calculated two grain sizes and mass fraction that give the best match with the data. Therefore, the sizes and mass fractions of grain sizes now become arbitrary fitting parameters. From the SEM image, the size of the smallest grain was found to be, about $0.063 \mu \mathrm{m}$ in diameter, which is close to the smallest value used in the bimodal distribution.

From the SEM images, it was clear that there were not just two grain sizes. Using the Visilog ${ }^{\mathrm{TM}}$ imaging software, GPM models with 10, 15, 20 and 30 grain sizes were used. Using 15 grain sizes gave better results than 10 sizes while 20 and 30 sizes showed little improvement over the 15 size GPM. Thus, 15 grains sizes GPM was used to describe $60 \%, 80 \%$ sorbent conversion profile and the results compared with the experimental data were good. However, they were worse than the arbitrary bimodal distribution. 
From the Visilog ${ }^{\mathrm{TM}}$ software results, the sphericity $(\varphi)$ of grains was estimated. The sphericities, obtained from evaluating 2-D images, ranged from about 0.2 to around 0.7 for 10, 15, 20 and 30 grain sizes distribution. The sphericity $(\varphi)$ for 10, 15, 20 and 30 grain size distributions, for the two smallest grains were always larger than 0.5 while the rest were smaller than 0.5 .

A modified grain size equal to $\varphi \cdot R$ was used in GPM, but this modification degraded the prediction. The flat plate-shaped GPM was also used instead of spherical grains, when $\varphi<0.5$, but this modification also gave poor predictions. Because the sphericity of most grains in the four grain size distributions (10, 15, 20 and 30) are less than 0.5 , it might be concluded that the shape of all grains is more close to a disc than to a sphere. However when the flat plate-shaped GPM was used for all grains, the result did not compare well with the experimental data.

The bimodal grain size model does not seem to represent well the actual distribution of grains within the sorbents. Nevertheless, the predictions of the bimodal GPM give excellent agreement with the experimentally determined conversion-time profiles for both the $60 \%$ and $80 \%$ sorbents and appear to be the best way to describe the conversion histories of these sorbents. 


\section{RECOMMENDATIONS FOR FUTURE WORK}

The modeling of gas-solid reactions can generally be classified into two categories: reaction on the surface of nonporous grains and reaction taking place on complicated porous structure. For the current work, the former models were used. Future work in the modeling of these complex situations, using the overlapping grain model may be worthwhile. 


\section{REFERENCES}

1. Grindley T. and Steinfeld G., Development and Testing of Regenerable Hot Coal Gas Desulfurization on Sorbents, Final Report DOE/MC/16545-1125, 1981.

2. Gibson J.B. and Harrison D.P., The Reaction Between Hydrogen Sulfide and Spherical Pellets Zinc Oxide, Ind. Eng. Chem. Process Des. 19, 231-237, 1980.

3. Focht G.D., Ranade P.V. and Harrison D.P., High-temperature desulfurization using zinc ferrite: reduction and sulfidation kinetics, Chem.Engin Sci. 43, 3005-3013, 1988.

4. Ranade P.V. and Harrison D.P, The variable property grain model applied to the zinc oxide-hydrogen sulfide reaction Chem. Engin. Sci. 36, 1079-1089, 1981.

5. Westmoreland P.R., Gibson J.B.and Harrison D.P., Comparative Kinetics of HighTemperature Reaction Between $\mathrm{H}_{2} \mathrm{~S}$ and Selected Metal Oxides, Environmental Sci. \& Technol., 11, 5, 488-491, 1977.

6. Efthimiadis E. and Sotirchos V.S., Reactivity Evolution During Sulfidation of Porous Zinc Oxide, Chem.Engin Sci. 48, 829-843, 1993.

7. Pineda M., Palacios J.M., Garcia E., Cilleruelo C., and Ibarra J.V., Modeling of Performance of Zinc Ferrites as High-Temperature Desulfurizing Sorbents In a FixedBed Reactor, Fuel, 76, 567-573, 1997.

8. Lew S., Sarofim A.F. and Flytzani M.-Stephanopoulos, Sulfidation of Zinc Titanate and Zinc Oxide Solids, Ind. Eng. Chem. Res., 31, 1890-1899, 1992.

9. Konttinen J.T., Zevenhoven C.A.P. and Hupa M.M., Hot Gas Desulfurization with Zinc Titanate Sorbents in a Fluidized Bed. 1. Determination of Sorbent Particle Conversion Rate Model Parameters, Ind. Eng. Chem. Res., 36, 2332-2339, 1997. 
10. Jothimurugesan K. and Harrison D.P., Reaction between H2S and Zinc OxideTitanium Oxide Sorbents: 2. Single-Pellet Sulfidation Modeling, Ind. Eng. Chem. Res., 29, 1167-1172, 1990.

11. Lew S., Sarofim A.F. and Flytzani-Stephanopoulos M., Modeling of the Sulfidation of Zinc-Titanium Oxide Sorbents with Hydrogen Sulfide, AICHE., 38, 1161-1169, 1992.

12. Li Y., Guo H., Li C. and Zhang S., A Study on the Apparent Kinetics of H2S Removal Using a ZnO-MnO Desulfurizer, Ind. Eng. Chem. Res., 36, 3982-3987, 1990.

13. Flytzani-Stephanopoulos M. and Li Z, Kinetics of Sulfidation Reactions Between $\mathrm{H}_{2} \mathrm{~S}$ and Bulk Oxide Sorbents, NATO ASI Series, G42, 179-211, Edited by Atimtay and Harrison, Springer-Verlag, Berlin, 1998.

14. Szekely J., Evans J.W. and Sohn H.Y., Gas-Solid Reactions, Academic Press, New York, 1976.

15. Shen J. and Smith J.M., Diffusional Effects in Gas-Solid Reactions, I\&EC FUNDAMENTALS, 4, 293-301, 1965.

16. Sotirchos. S.V. and Yu H.C., Overlapping Grain Models for Gas-Solid Reactions with Solid Product, Ind. Eng. Chem. Res., 27, 836, 1988.

17. Bartlett R. W., Krishnan N.G. and Van Hecke M.C., Mircrograin Models of Reacting Porous Solids with Approximations to Logarithmic Solid Conversion, Chem.Engin Sci. 28, 2179-2186, 1973. 
18. Lindner. B. and D. Simonsson, Comparison of Structure Models for Gas-Solid Reactions in Porous Solids Undergoing Structure Changes, Chem. Eng. Sci., 36,1519, 1981.

19. Bhatia S.K. and Perlmutter D.D., AICHE Journal, 26, 379-386, 1980.

20. Satterfield C.N., Heterogeneous Catalysis in Industrial Practice, $2^{\text {nd }}$ ed., Krieger Publishing Co., Malabar, FL, 1996.

21. Kunii D. and O. Levenspiel, $2^{\text {nd }}$ ed., Butterworth-Heinemann, Boston, MA, 1991.

22. Hirschfelder J.O., Bird R.B. and E.L. Spotz, Chem. Revs., 44, 205-231, 1949. 


\section{APPENDIX}

$\underline{\text { Appendix I: Shrinking Core Model for flat plate shaped particles }}$

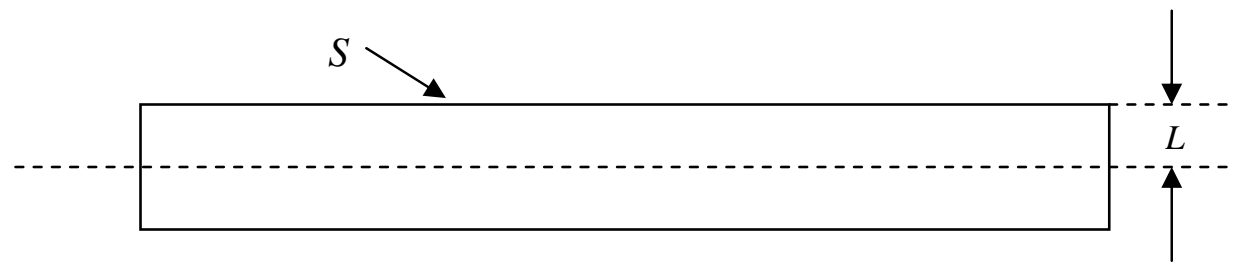

Here, $S$ is the surface area of the flat plate and $L$ is the half thickness of the flat plate.

The three steps involved in modeling the overall reaction, and the corresponding rates, are as follows.

1. Diffusion through the external gas film surrounding the particle (external mass transfer resistance) can be expressed as:

$$
-r_{A}=2 S \cdot k_{g}\left(C_{A b}-C_{A s}\right)
$$

2. Diffusion through the product layer (ash layer) based on the assumption of equimolar counter diffusion can be expressed as:

$$
-r_{A}=\frac{2 S \cdot D_{e}}{L-L_{c}}\left(C_{A s}-C_{A c}\right)
$$

3. Chemical reaction at the interface can be expressed as:

$$
-r_{A}=2 S \cdot k C_{A c}
$$

The rate of reaction at a given time $t$ (when the position of the reaction interface is at $L_{c}$ ) is given by combining Equations (A.1), (A.2), (A.3) and eliminating the interface concentrations: 


$$
-r_{A}=\left(\frac{1}{k_{g}}+\frac{L-L_{c}}{D_{e}}+\frac{1}{k}\right)^{-1} 2 S \cdot C_{A b}
$$

Because the solid product layer ( $\mathrm{ZnS}$ ) does not have the same volume as the solid reactant $(\mathrm{ZnO})$ consumed, a change in particle size will occur. We use $Z_{v}$ to model into the SCM.

$$
Z_{v}=\frac{\text { Volume of ZnS formed }}{\text { Volume of ZnO consumed }}=\frac{S \cdot\left(L-L_{c}\right)}{S \cdot\left(L_{o}-L_{c}\right)}
$$

So,

$$
L=Z_{v}\left(L_{o}-L_{c}\right)+L_{c}
$$

Here, $L_{o}$ is the initial half thickness of the flat plate and $L_{c}$ is the half thickness of the unreacted flat plate.

For a flat plate particle, the fractional conversion $(X)$ of the solid reactant is related to $L_{c}$ by:

$$
X=1-\frac{L_{c}}{L_{o}} \Rightarrow\left(L_{o}-L_{c}\right)=L_{o} \cdot X
$$

And, $\quad-r_{A}=\frac{1}{M_{Z n O}} \frac{d X}{d t}$

Substituting Equation (A.5) and (A.6) into Equation (A.4) and integrating, the following relationship between conversion and time is obtained:

$$
\frac{1}{k_{g}} X+\frac{Z_{v} L_{o}}{2 D_{e}} X^{2}+\frac{1}{k}=\frac{C_{A b} M_{\mathrm{ZnO}}}{\rho_{\mathrm{ZnO}} \cdot L_{o}} t
$$


Rearranging Equation (A.7) gives:

$$
t=t_{\text {total }}=t_{\text {film }}+t_{\text {ash }}+t_{\text {reac }}
$$

where, $\quad t_{\text {film }}=\tau_{\text {film }} X ; \tau_{\text {film }}=\frac{\rho_{Z n O} L_{o}}{k_{g} C_{A b} M_{Z n O}}$

$$
\begin{gathered}
t_{\text {ash }}=\tau_{\text {ash }} X^{2} ; \tau_{\text {ash }}=\frac{\rho_{\mathrm{ZnO}} L_{o}^{2} Z_{v}}{2 D_{e} C_{A b} M_{\mathrm{ZnO}}} \\
t_{\text {reac }}=\tau_{\text {reac }} X ; \tau_{\text {reac }}=\frac{\rho_{\mathrm{ZnO} O} L_{o}}{k C_{A b} M_{\mathrm{ZnO}}}
\end{gathered}
$$

